\title{
Automorphe Formen und L-Funktionen
}

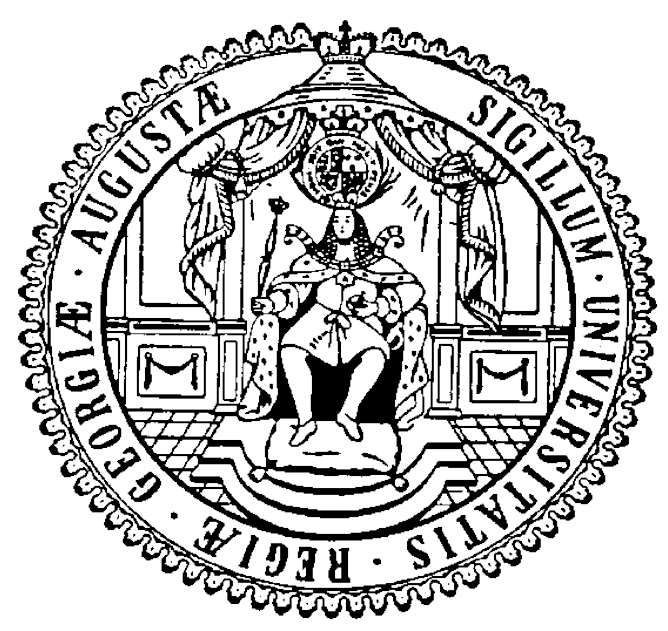

Dissertation

zur Erlangung des mathematisch-naturwissenschaflichen Doktorgrades

"Doctor rerum naturalium"

der Georg-August Universität Göttingen

im Promotionsstudiengang "Mathmatical Sciences (Ph.D)"

der Georg-August University School of Science (GAUSS)

Vorgelegt von

Rebecca Ulrike Möser

aus Göttingen 
Betreuungsausschuss:

Professor Dr. Valentin Blomer,

Mathematisches Institut,

Universität Bonn

Professor Dr. JÖRG BRÜDERN,

Mathematisches Institut,

Georg-August Universität

$\underline{\text { Mitglieder der Prüfungskommission }}$

Referent: Professor Dr. VAlentin Blomer

Korreferent: Professor Dr. JÖRG BRÜDERN

Weitere Mitglieder der Prüfungskommission:

Professor Dr. Preda Mihailescu,

Mathematisches Institut,

Georg-August Universität

Professor Dr. DAmaris SchindleR,

Mathematisches Institut,

Georg-August Universität

Professor Dr. Russell Luke,

Institut für Numerische und Angewandte Mathematik,

Georg-August Universität

Juniorprofessor Dr. DANiEl Rudolf,

Institut für Mathematische Stochastik,

Georg-August Universität

Tag der mündlichen Prüfung: Montag 01.03.2021 


\section{Acknowledgements}

First of all I want to thank Prof. Dr. Valentin Blomer for his support during my final thesis and his time for detailed feedback. Further I sincerely thank Prof. Dr. J. Brüdern for his advice. Moreover I thank my husband Theo and my office mates Fabian and Burkhard for advice, emotional support and consistent encouragement, my sister Johanna for supplying me with the best cakes and pies. Further I thank my grandfather Prof. Dr. Wolfgang Fischer and my uncle Daniel for proofreading and stylistic advice. Of course I thank my parents for their support. Last but not least I thank my son Johann without whom there would have been a lot less laughter during the working times. 


\section{Contents}

\begin{tabular}{llr}
\hline 1 & Introduction & 1
\end{tabular}

1.1 Fourth Power Moment of Hecke $L$-functions $\ldots \ldots \ldots \ldots$. . . . . . . . . . . . . . . . . . 4

1.1 .1 Results . . . . . . . . . . . . . . . . . . . . . . . . . 4 4

1.1 .2 Method of Proof . . . . . . . . . . . . . . . . . . . . . 5

$1.2 \quad$ Second Power Moment of Rankin-Selberg $L$-functions . . . . . . . . . . . . . . . . . . . 6

1.2 .1 Result . . . . . . . . . . . . . . . . . . . . . . . . . . . . 6

1.2 .2 Method of Proof $\ldots \ldots \ldots \ldots \ldots \ldots \ldots \ldots$

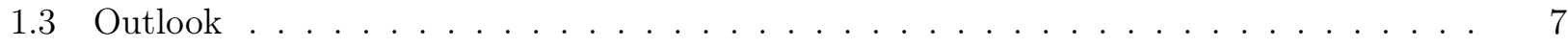

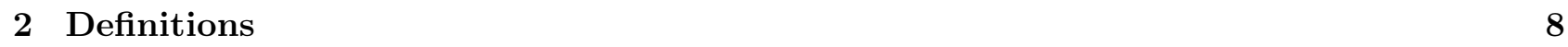

3 Fourth Power Moment of Hecke $L$-functions over Totally Real Number Fields 10

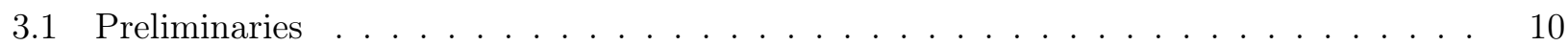

3.1 .1 Special Functions $\ldots \ldots \ldots \ldots \ldots \ldots$. . . . . . . . . . . . . . . . . . . . . . . .

Eisenstein Series . . . . . . . . . . . . . . . . . . . . . . 15

3.1 .2 Eigenvalues and Hecke Operators . . . . . . . . . . . . . . . . . . . 17

Geometric Sum Formula . . . . . . . . . . . . . . . . . . . . . . . . . . . . . 21

Hecke Series . . . . . . . . . . . . . . . . . . . . . . 23

3.2 Spectral Decomposition of The Fourth Moment of $\zeta_{K} \ldots \ldots \ldots \ldots$. . . . . . . 26

3.2 .1 Initial Reduction . . . . . . . . . . . . . . . . . . . . . . . . 26

3.2 .2 Kloosterman Sums . . . . . . . . . . . . . . . . . . . . . . . . . . . . . . . . . 30

$3.2 .3 \quad$ Spectral Decomposition of $\mathcal{B}_{m} \ldots \ldots \ldots \ldots \ldots \ldots$

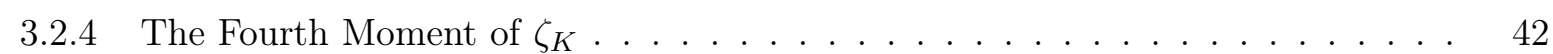

3.3 A Bound for The Fourth Moment . . . . . . . . . . . . . . . . . . . . . . . . . . . . 48

3.3 .1 Error Term . . . . . . . . . . . . . . . . . . . . . . . . . . . . 48

3.3 .2 Main Term . . . . . . . . . . . . . . . . . . . . . . . . 52

4 Second Moment of Rankin-Selberg $L$-functions over Totally Real Number Fields 56

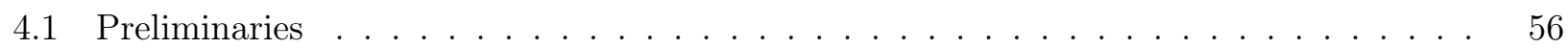

4.1 .1 Hilbert Modular Forms $\ldots \ldots \ldots \ldots \ldots \ldots$

$4.1 .2 \quad$ Atkin-Lehner Theory . . . . . . . . . . . . . . . . . . . . . 57

4.1 .3 Rankin-Selberg $L$-functions . . . . . . . . . . . . . . . . . . . . . . . . . . . . . . 59

4.1 .4 Voronoi's Formula $\ldots \ldots \ldots \ldots$. . . . . . . . . . . . . . . . . . 62

4.1 .5 Further Results . . . . . . . . . . . . . . . . . . . . . . . . . . . . . . . . . 64

4.2 Proof of Theorem $\mid 1.2 .1 \ldots \ldots \ldots \ldots \ldots$

$4.2 .1 \quad$ Initial Reduction $\ldots \ldots \ldots \ldots$. . . . . . . . . . . . . . . . . . . . . . . . 66 
$4.2 .2 \quad$ Application of Voronoi's Summation Formula $\ldots \ldots \ldots \ldots$

\begin{tabular}{lll}
\hline 5 & Appendix & $\mathbf{7 7}$
\end{tabular}

5.1 Whittaker Functions . . . . . . . . . . . . . . . . . . . . 77

5.2 Bessel Functions $\ldots \ldots \ldots \ldots \ldots \ldots \ldots$ 



\section{Chapter 1}

\section{Introduction}

Estimating moments of families of $L$-functions over number fields is a central problem in number theory.

On the one hand, such estimates serve as tools: An upper bound for the second power moment of Rankin-Selberg $L$-functions combined with a lower bound of the first moment can be used to show non-vanishing of Rankin-Selberg $L$-functions at the central point.

In addition, an upper bound for the fourth power moment of Hecke $L$-functions is used in [D89] to estimate the number of zeros of Hecke $L$-functions with a certain distance to the critical line.

On the other hand, these moments are also seen as natural objects that have to be studied independently because they illuminate the structure of the family and expose beautiful symmetries.

In Chapter 3 of this thesis we will establish an asymptotic formula for the fourth moment of Hecke $L$-functions and in Chapter 4 an upper bound for the second moment of Rankin-Selberg $L$-functions.

Several results on moments of $L$-functions are well known. A classical approach to compute moments of $L$-functions is to start with the application of an approximate functional equation. Then, one can for example apply the Montgomery-Vaughan identity or a trace formula and other estimates. This works very well for the second and the fourth power moment. For higher moments, the error terms of the approximate functional equation and of other methods become too large.

A special $L$-function that received a lot of attention is the Riemann $\zeta$-function. Ingham proved 1928 in I28 the asymptotic formula

$$
\int_{0}^{T}\left|\zeta\left(\frac{1}{2}+i t\right)\right|^{4} d t=a_{4} T(\log T)^{4}+\mathcal{O}\left(T(\log T)^{3}\right) .
$$

In 1979 Heath-Brown ( $[$ HB79 $)$ improved this by showing

$$
\int_{0}^{T}\left|\zeta\left(\frac{1}{2}+i t\right)\right|^{4} d t=T p_{4}(\log T)+\mathcal{O}\left(T^{\frac{7}{8}+\varepsilon}\right) .
$$

The coefficients of the fourth degree polynomial $p_{4}$ can be explicitly computed.

Motohashi proved an exact formula for the smoothed version of the fourth moment. In his paper M97] 
he considered the integral

$$
\int_{-\infty}^{\infty}\left|\zeta\left(\frac{1}{2}+i t\right)\right|^{4} g(t) d t
$$

where $g$ is a smooth function, of rapid decay outside an interval $[0, T]$. To deduce an asymptotic formula he considered the integral

$$
\int_{-\infty}^{\infty} \zeta\left(z_{1}+i t\right) \zeta\left(z_{2}+i t\right) \zeta\left(z_{3}-i t\right) \zeta\left(z_{4}-i t\right) g(t) d t
$$

He rewrote this as sums over Kloosterman sums and used a spectral decomposition to get a sum containing a residual contribution, a cuspidal contribution and a contribution of Eisenstein series. The obtained sum has a continuation to a neighborhood of the point $\left(z_{1}, z_{2}, z_{3}, z_{4}\right)=\left(\frac{1}{2}, \frac{1}{2}, \frac{1}{2}, \frac{1}{2}\right)$. To obtain the asymptotic formula for the fourth moment, he estimated the cuspidal and the Eisenstein contribution and computed asymptotics for the contribution of the residues. This improves HeathBrown's error term to

$$
\int_{0}^{T}\left|\zeta\left(\frac{1}{2}+i t\right)\right|^{4} d t=T p_{4}(\log T)+\mathcal{O}\left(T^{\frac{2}{3}}(\log T)^{8}\right) .
$$

These asymptotic formulas improve the standard estimate

$$
\int_{0}^{T}\left|\zeta\left(\frac{1}{2}+i t\right)\right|^{4} d t \ll T(\log T)^{4},
$$

see for example [T51, Theorem 7.16].

There are further results on moments of the Riemann $\zeta$-function, for example those given by Motohashi, Beineke and Bump. In his paper [M07] Motohashi considers integrals of the form

$$
\int_{1}^{\infty}\left|\zeta\left(\frac{1}{2}+i t\right)\right|^{4}\left|\sum_{n \leq N} a_{n} n^{-\frac{1}{2}-i t}\right|^{2} g(t) d t .
$$

Furthermore, Beineke and Bump considered the second moment of the Riemann $\zeta$-function in parts of the critical strip in their paper BB04].

Moreover there are some investigations on moments of Dirichlet $L$-functions and modular forms. For example, in Y06 Young considered the fourth moment of Dirichlet $L$-functions. Blomer, Milićević, Fouvry, Kowalski and Michel give asymptotic formulas for moments of twisted $L$-functions and modular forms in the papers BM15] and BFKMM17.

The Rankin-Selberg $L$-functions were for example considered by Blomer B11]. He proved the upper bound

$$
\sum_{g \in B_{k}(1)}\left|L\left(f \otimes g, \frac{1}{2}\right)\right|^{2} \ll k^{1+\varepsilon}
$$

for the second power moment of Hecke eigenforms of level one, by introducing an unfolding method. 
In my masters thesis I generalized this result for $f$ and $g$ newforms of weight $k$ and level $N$. The estimate is given by

$$
\sum_{f \in B_{k}^{\sharp}(N)} \frac{\left|L\left(f \otimes g, \frac{1}{2}\right)\right|^{2}}{L\left(\operatorname{sym}^{2} f, 1\right)} \ll(N k)^{1+\varepsilon},
$$

simultaneously in $N$ and $k$. Since the space of cusp forms has dimension $(N k)^{1+o(1)}$ this is the best possible result.

Kowalski, Michel and Vanderkam proved an upper bound for the Rankin-Selberg $L$-functions and all their derivatives in KMV00.

There are also some results for general number fields $K$.

The Hecke $L$-series of a number field $K$ with class number one attached to a Größencharacter $\lambda_{\underline{\nu}}$ is, for $\operatorname{Re} s>1$, defined by

$$
\zeta_{K}(s, \underline{\nu})=\sum_{\mathfrak{a}=(a)} \lambda_{\underline{\nu}}(a)(N \mathfrak{a})^{-s}
$$

for the notation see Section 3.1. The Dedekind $\zeta$-function is the Hecke $L$-function attached to the trivial character, i.e. $\lambda_{\underline{\nu}} \equiv 1$.

An analogue to the classical estimate (1.0.1) for the Riemann $\zeta$-function was proven by Duke in [D89], namely he proved the estimate

$$
\sum_{|m| \leq T} \int_{-T}^{T}\left|\zeta_{K}\left(\frac{1}{2}+i t, \chi \lambda^{m}\right)\right|^{4} d t \ll T^{n}(\log T)^{A}
$$

for a general number field $K$ with degree $n$. The exponent $A$ depends only on $K$. Here $\chi$ is a character of the class group and the character $\lambda^{m}=\lambda_{1}^{m_{1}} \cdots \lambda_{n}^{m_{n}}$ where $\left\{\lambda_{j}\right\}$ is a basis for the torsion-free Hecke characters.

For some number fields, Bruggeman and Motohashi proved a decomposition of the fourth moment of the Dedekind $\zeta$-function into third moments analogous to that of Motohashi in [M97]. They considered the integral

$$
\int_{-\infty}^{\infty}\left|\zeta_{K}\left(\frac{1}{2}+i t\right)\right|^{4} g(t) d t
$$

where $g$ is an entire function, of rapid decay in each fixed horizontal strip.

In [BM01] they compute the spectral decomposition of the smoothed fourth moment of Dedekind $\zeta$-functions for a real quadratic number field $K$ of class number one. For the Gaussian number field this is done in their paper BM02].

A more general result was proved by Nelson. In his paper N20 he spectrally decomposes a third moment of $L$-series in an abstract way into a fourth moment for general number fields, reversing the decomposition of BM01 and BM02.

Similarly, moments for the Dedekind $\zeta$-function and Hecke $L$-functions are for example investigated by 
Heap in [H12 and [H19]. He considered the $2 k$-th moment of the Dedekind $\zeta$-function and the twisted second moment of the Dedekind $\zeta$-function and an $L$-function for quadratic fields. Watt, in WW13, gave an estimate analogous to Motohashi's result in [M07. Topacogullari computed an asymptotic formula for Hecke $L$-functions in [To19]. Thorner also considered the fourth moment of Hecke $L$-functions and computed an upper bound in [Th19]. Diaconu, Garrett and Goldfeld considered moments of twisted $L$-functions in their papers DG09 and DGG11.

Coleman in [C92] used the result of Duke [D89] to examine the local distribution of prime ideals in narrow ideal classes within any number field.

The Rankin-Selberg $L$-functions over number fields were considered by Liu and Masri and by Hamieh and Tanabe. Liu and Masri proved in their paper [LM14] an asymptotic formula for the first moment and an upper bound for the second power moment for Hilbert modular forms of parallel weight $2 k$ and level one attached to a totally real number field of degree $n$ with class number one. They get the result

$$
\sum_{g \in B_{2 k}}\left|L\left(f \otimes g, \frac{1}{2}\right)\right|^{2} \ll k^{n+\varepsilon}
$$

They adapted the unfolding method of [B11] and use this estimate in combination with an asymptotic formula for the first moment to show non-vanishing of the Rankin-Selberg $L$-function at the central point.

Hamieh and Tanabe generalized this result in their paper [HT20]. They fix a Hilbert modular form $f$ of parallel weight $k$ and level $\mathfrak{n}$, sum over a basis of primitive forms of parallel weight $k$ and level one and prove the bounds

$$
\sum_{g \in B_{k}}\left|L\left(f \otimes g, \frac{1}{2}\right)\right| \gg k, \quad \sum_{g \in B_{k}}\left|L\left(f \otimes g, \frac{1}{2}\right)\right|^{2} \ll k(\log k)^{c} .
$$

Hamieh and Tanabe also use their results to prove non-vanishing of Rankin-Selberg $L$-functions.

\subsection{Fourth Power Moment of Hecke $L$-functions}

\subsubsection{Results}

In Chapter 3 we deduce a result similar to that of Motohashi [M97] for a totally real number field $K$ of degree $d$ with class number one. We compute an explicit spectral decomposition for the fourth power moment of Hecke $L$-functions over a totally real number field with class number one and deduce from this an asymptotic formula.

In such a number field, the Größencharacters are given by

$$
\lambda_{\underline{\nu}}(n)=\left|\frac{n_{1}}{n_{d}}\right|^{i \nu_{1}} \cdots\left|\frac{n_{d-1}}{n_{d}}\right|^{i \nu_{d-1}},
$$

where $\underline{\nu}$ is a $(d-1)$-dimensional vector in $\mathbb{R}^{d-1}$ that satisfies certain conditions. We write $\Lambda^{d-1}$ for the lattice consisting of these vectors. Then a Hecke $L$-series is defined as

$$
\zeta_{K}(s, \underline{\nu})=\sum_{\mathfrak{a}=(a)} \lambda_{\underline{\nu}}(a)(N \mathfrak{a})^{-s}
$$

Using the spectral decomposition of sums over Kloosterman sums given in BMP01, we follow the 
steps of [BM01] to get a spectral decomposition for the average value of Hecke $L$-functions, namely

\section{(1.1.1) Theorem}

Let $K$ be a totally real number field of degree $d$ and class number one, and let $K$ be such that every ideal has a totally positive generator. Let $g(t)$ and $\omega\left(\nu_{1}, \ldots, \nu_{d-1}\right)$ be entire, and of rapid decay in every fixed horizontal strip for every variable.

Then, we have

$$
\begin{aligned}
& \sum_{\underline{\nu} \in \Lambda^{d-1}} \omega(\underline{\nu}) \int_{-\infty}^{\infty}\left|\zeta_{K}\left(\frac{1}{2}+i t, \underline{\nu}\right)\right|^{4} g(t) d t=M_{K}(g, \omega) \\
& +\sum_{V} a_{V} \eta_{V}(1) H_{V}\left(\frac{1}{2}\right)^{3} \Lambda_{V}(g, \omega)+2^{d} \sqrt{\left|D_{K}\right|} \sum_{\underline{\nu} \in \Lambda^{d-1}-\infty} \int_{-\infty}^{\infty} \frac{\left|\zeta_{K}\left(\frac{1}{2}+i t, \underline{\nu}\right)\right|^{6}}{\left|\zeta_{K}(1+2 i t, 2 \underline{\nu})\right|^{2}} \Xi_{\underline{\nu}}(t ; g, \omega) d t
\end{aligned}
$$

where $D_{K}$ is the discriminant of $K$, and $V$ runs over the cuspidal subspaces of the Hilbert space of all $\Gamma$-automorphic functions on $G$, see (3.1.28), where $G=\mathrm{PSL}_{2}(\mathbb{R})^{d}$ and $\Gamma=\mathrm{PSL}_{2}(\mathfrak{o})$ is the Hilbert modular group. The functions $\Lambda_{V}(g, \omega)$ and $\Xi_{\underline{\nu}}(t ; g, \omega)$ are integral transforms of $g$ and $\omega$. The term $M_{K}(g, \omega)$ comes from the residual contributions, it is defined in Theorem 3.2.5. The functions $a_{V}, \eta_{V}$ and the Hecke series $H_{V}$ are defined in Section 3.1.2.

We use this decomposition to obtain an asymptotic formula for the fourth moment of Hecke $L$-series:

\section{(1.1.2) Theorem}

Let $K$ be a totally real number field of degree $d$ and class number one, and let $K$ have fundamental units ${ }_{j} \epsilon$ such that every ideal has a totally positive generator. Let $G$ and $\Omega$ be two entire functions that are of rapid decay and satisfy the estimates

$$
\begin{aligned}
& G^{(j)}(x) \ll_{A, j}(1+|x|)^{-A}, \\
& \frac{\partial^{j_{1}}}{\partial y_{1}^{j_{1}}} \cdots \frac{\partial^{j_{d-1}}}{\partial y_{d-1}^{j_{d-1}}} \Omega(\underline{y}) \ll_{A, \underline{j}}\left(1+\left|y_{1}\right|+\cdots+\left|y_{d-1}\right|\right)^{-A}
\end{aligned}
$$

with $A>2$ for all $j, j_{1}, \ldots, j_{d-1} \in \mathbb{N}_{0}$.

In addition, we define $g(t)=G\left(\frac{t}{T}\right)$ and $\omega(\underline{\nu})=\Omega\left(\frac{\nu_{1}}{T}, \ldots, \frac{\nu_{d-1}}{T}\right)=\Omega(\underline{\nu} / T)$.

Then, we have

$$
\sum_{\underline{\nu} \in \Lambda^{d-1}} \omega(\underline{\nu}) \int_{\mathbb{R}}\left|\zeta_{K}\left(\frac{1}{2}+i t, \underline{\nu}\right)\right|^{4} g(t) d t=T^{d} P_{3+d}(\log T)+\mathcal{O}\left(T^{d-1+\varepsilon}+T^{\frac{d}{2}+d \theta}\right),
$$

where $P_{3+d}$ is a polynomial of degree $3+d$, and $\theta$ is an admissible exponent for the Ramanujan conjecture.

By BB11, one may take $\theta=\frac{7}{64}$.

\subsubsection{Method of Proof}

We give a brief sketch of the proof of Theorem 1.1.1 and Theorem 1.1.2. The proof of Theorem 1.1.1 follows the paper BM01 of Bruggeman and Motohashi. 
We consider the more general expression

$$
\sum_{\underline{\nu} \in \Lambda^{d-1}} \omega(\underline{\nu}) \int_{-\infty}^{\infty} \zeta_{K}\left(z_{1}+i t, \underline{\nu}\right) \zeta_{K}\left(z_{2}+i t, \underline{\nu}\right) \zeta_{K}\left(z_{3}-i t,-\underline{\nu}\right) \zeta_{K}\left(z_{4}-i t,-\underline{\nu}\right) g(t) d t
$$

which is absolutely convergent for $\operatorname{Re} z_{j}>1$, and has a meromorphic continuation to $\mathbb{C}^{4}$. In particular, it is regular at the central point $\left(z_{1}, z_{2}, z_{3}, z_{4}\right)=\left(\frac{1}{2}, \frac{1}{2}, \frac{1}{2}, \frac{1}{2}\right)$ for which we want to compute the asymptotic formula.

We have to rewrite this expression as sums over Kloosterman sums. To this end we use a relation, namely (3.1.10), between the product of Hecke $L$-series and divisor functions, and several reformulations as well as the Ramanujan expansion in Section 3.2.1. Then, the functional equation of a resulting factor leads to an expression in terms of Kloosterman sums in Section 3.2.2. In Section 3.1 .2 we state the spectral decomposition that we apply in Section 3.2 .3 to our expression of Kloosterman sums. Finally we expand this decomposition to a region around the central point $\left(z_{1}, z_{2}, z_{3}, z_{4}\right)=\left(\frac{1}{2}, \frac{1}{2}, \frac{1}{2}, \frac{1}{2}\right)$.

To prove Theorem 1.1.2 we use the decomposition stated in Theorem 1.1.1 and compute the terms separately.

The summand $M_{K}(g, \omega)$ is regular but consists of summands that individually have poles. In Lemma 3.3.3 we rewrite it into an expression that can be handled more easily. Then Stirling's formula and trivial operations give an asymptotic formula.

To estimate the two remaining summands, we estimate the integral transformations $\Lambda_{V}(g, \omega)$ and $\Xi_{\nu}(t ; g, \omega)$ of $g$ and $\omega$. Further we use estimates on $H_{V}$ and the sum over $a_{V}$ and $\eta_{V}$ stated in Section 3.1 .2 for the cuspidal contribution. Estimates for the Hecke $L$-functions appearing in the Eisenstein contribution are stated in Section 3.1. Combining these bounds, we find that the cuspidal and the Eisenstein contribution enter the error term.

\subsection{Second Power Moment of Rankin-Selberg $L$-functions}

\subsubsection{Result}

In Chapter 4 we prove an upper bound for the second power moment of Rankin-Selberg $L$-functions of Hecke eigenforms attached to a totally real number field $K$ of degree $d$ and class number one.

In $K$, we fix a prime ideal $\mathfrak{q}$ and an integral ideal $\mathfrak{c}$ that is either coprime to $\mathfrak{q}$ or equal to $\mathfrak{q}$. Next, we pick some primitive Hilbert modular form $g \in S_{\underline{k}}(\mathfrak{q})$, and sum over a basis of primitve Hilbert modular forms of level $\mathfrak{c}$.

\section{(1.2.1) Theorem}

Let $K$ be a totally real number field of degree $d$ and class number one. Let $\mathfrak{q}$ be a prime ideal and $\mathfrak{c}$ be a squarefree integral ideal either coprime or equal to $\mathfrak{q}$, let $\underline{k}=(k, \ldots, k) \in \mathbb{N}^{d}$, with $k$ large relatively to $N \mathfrak{c}$, more precisely $k>(N \mathfrak{q} \mathfrak{c})^{\varepsilon}$. Further let $g \in S_{\underline{k}}^{\sharp}(\mathfrak{q})$ be a newform with $C_{g}(\mathfrak{q}) \neq 0$ and $\mathcal{B}_{\underline{k}}^{\sharp}(\mathfrak{c})$ be a basis of primitive Hilbert modular forms of weight $\underline{k}$ and level $\mathfrak{c}$. Then for $\varepsilon>0$ the following estimate holds:

$$
\frac{\Gamma(k-1)^{d}}{(4 \pi)^{d(k-1)}\left|D_{K}\right|^{\frac{1}{2}}} \sum_{f \in \mathcal{B}_{\underline{\underline{k}}}^{\sharp}(\mathfrak{c})} \frac{\left|L\left(f \otimes g, \frac{1}{2}\right)\right|^{2}}{\langle f, f\rangle} \ll(N \mathfrak{c})^{\varepsilon} k^{d \varepsilon} .
$$


The implicit constant depends on $K$ and if $(\mathfrak{q}, \mathfrak{c})=1$ on $N \mathfrak{q}$. The inner product $\langle f, f\rangle$ is defined in 4.1.2.

\subsubsection{Method of Proof}

We give a brief sketch of the proof.

To begin with, we apply an approximate functional equation, as in the classical approach. Extending the sum to a basis of the whole space and writing out the square allows us to apply the Petersson trace formula L03, (8)] to the sum over $f \in \mathcal{B}_{\underline{k}}(\mathfrak{c})$.

This yields a diagonal term and an off-diagonal term. The second one contains a sum over a product of Kloosterman sums and a product of Bessel $J$-functions.

The diagonal term can be estimated trivially. By the decay properties of the Bessel $J$-function and the the approximate functional equation, we can also estimate large parts of the off-diagonal term trivially. The remaining sum is over ideals $\mathfrak{m}_{1}, \mathfrak{m}_{2}$ and $c \in \mathfrak{c}$ with small norm. We apply a Voronoi summation formula to the sum over $\mathfrak{m}_{1}$ and estimate the resulting expression carefully to prove Theorem 1.2.1.

\subsection{Outlook}

Naturally, the question arises, whether one can expect similar results for more general fields.

This is the case, albeit with a lot more technical work.

For the computation of the asymptotic formula of the fourth power moment, the arguments used to deduce the representation in terms of Kloosterman sums are based on the class number one assumption. Thus for a real number field with arbitrary class number this step must be modified.

For number fields that are not totally real, the explicit spectral decomposition is not in the literature. For the Gaussian number field this is done by Bruggeman and Motohashi in their paper [BM02]. One expects that an explicit spectral decomposition formula can be proven in general. But until now there is no asymptotic formula. One expects that this could also be done as in the present work, but the combinatorics are more complicated.

To estimate the second moment of Rankin-Selberg $L$-functions, the Voronoi summation formula is based on the class number one assumption. Thus, for a real number field with arbitrary class number a new version of this has to be proven, a Kloosterman sum for totally real number fields of arbitrary class number is given in HT16.

The restriction on the level should be possible to remove. For newforms of squarefree level and trivial character, this should follow by the same steps. For more general level and character following the steps of [L79] should give a functional equation. 


\section{Chapter 2}

\section{Definitions}

Before we prove our results, we specify the number field we work in and define some notation.

Let $K$ be a totally real number field over $\mathbb{Q}$ with degree $d$, of narrow class number one. Further let $D_{K}$ denote the discriminant of $K$. We denote by $\mathfrak{o}$ the ring of integers of $K$ and by $\mathfrak{o}^{\times}$the group of units. For an ideal $\mathfrak{n}$ let $\mathfrak{n}^{*}=\mathfrak{n} \backslash\{0\}$, in particular $\mathfrak{o}^{*}=\mathfrak{o} \backslash\{0\}$.

Let $\sigma_{j}$ be the embeddings $K \rightarrow \mathbb{R}$. We identify $n \in K$ with the corresponding vector $\left(n_{1}, \ldots, n_{d}\right) \in \mathbb{R}^{d}$ where $n_{j}=\sigma_{j}(n)$.

We denote by ${ }_{j} \epsilon, j=1, \ldots, d-1$ a system of fundamental units of $K$. For sake of simplicity we assume that every ideal has a totally positive generator. We write $a \succ 0$ for an element $a \in K$ that is totally positive, i.e. $a_{j}>0$ for all $j=1, \ldots, d$.

We denote by $P(\epsilon)$ the product ${ }_{1} \epsilon \cdots_{d-1} \epsilon$, and by $R_{K}$ the regulator $\left|\operatorname{det}\left(\log \left({ }_{j} \epsilon\right)_{i}\right)_{i, j=1, \ldots, d-1}\right|$ of $K$.

There is a natural bijection between $\mathfrak{o} / \mathfrak{o}^{\times}$and the set $I=\{\mathfrak{a}=(a) \mid a \in \mathfrak{o}\}$ of integral ideals.

Let $\mathcal{F}$ be a fundamental domain of $\mathfrak{o}$ with respect to the multiplication by units. We may choose $\mathcal{F}$ such that every element $a \in \mathcal{F}$ satisfies $a \succ 0$ and $a_{j} \asymp a_{i}$ in $\mathbb{R}$ for all $j, i=1, \ldots, d$. Here $a \asymp b$ means $a \ll b$ and $b \ll a$.

In other words, every ideal $\mathfrak{a}$ has a representation $\mathfrak{a}=(a)$ where $a_{j} \asymp(N \mathfrak{a})^{\frac{1}{d}}$ by choosing $a \in \mathcal{F}$, see [HT16, Lemma 1.1].

As usual $N(\cdot)$ and $\operatorname{Tr}(\cdot)$ denote the norm and the trace of $K$.

In the following computations several vectors of dimension $d$ or $(d-1)$ appear. To have a short and readable notation we introduce the following conventions. Let $d^{\prime}=d$ or $(d-1)$. Then let $\underline{x}, \underline{y} \in \mathbb{R}^{d^{\prime}}$, $z \in \mathbb{C}$ and $\circ=+, \cdot,-$. We define the operations component wise:

$$
\begin{aligned}
& d \underline{x}=d x_{1} \ldots d x_{d^{\prime}}, \\
& |\underline{x}|=\left(\left|x_{1}\right|, \ldots,\left|x_{d^{\prime}}\right|\right) \\
& \underline{x} \circ \underline{y}=\left(x_{1} \circ y_{1}, \ldots, x_{d^{\prime}} \circ y_{d^{\prime}}\right), \\
& \underline{x} \circ z=\left(x_{1} \circ z, \ldots, x_{d^{\prime}} \circ z\right), \\
& \underline{x} \underline{y}=x_{1}^{y_{1}} \cdots x_{d^{\prime}}^{y_{d^{\prime}}}, \\
& z^{\underline{y}}=z^{\operatorname{Tr}(\underline{y})}, \\
& \Gamma(\underline{x})=\prod_{j=1}^{d^{\prime}} \Gamma\left(x_{j}\right),
\end{aligned}
$$


and generalize the norm and the trace

$$
\begin{aligned}
& N(\underline{x})=x_{1} \cdots x_{d^{\prime}}, \\
& \operatorname{Tr}(\underline{x})=x_{1}+\cdots+x_{d^{\prime}} .
\end{aligned}
$$

For $x \in K$, we define

$$
e[x]=\exp (2 \pi i \operatorname{Tr}(x))
$$

Let $c, a \in \mathfrak{o}^{*}$ with $(a, c)=1$, and denote by $a^{*}$ the inverse of $a$ modulo $c$, in other words $a a^{*} \equiv 1$ $(\bmod c)$. Then the Kloosterman $\operatorname{sum} S_{K}(m, n ; c)$ over $K$ is given by

$$
S_{K}(m, n ; c)=\sum_{\substack{a \bmod c \\(a, c)=1}} e\left[\frac{a m+a^{*} n}{c}\right] .
$$

The Kloosterman sum satisfies the bound

$$
\left|S_{K}(m, n ; c)\right| \ll N((m, n, c))^{\frac{1}{2}} N(c)^{\frac{1}{2}+\varepsilon}
$$

where $(m, n, c)$ is the greatest common divisor and $\varepsilon>0$, see [HT20, (5.4)].

We denote by $\int_{(a)}$ the integral over the vertical line $\operatorname{Re} s=a$, and by $\widehat{f}(y)$ the Fourier transformation of $f$ given by the integral

$$
\widehat{f}(\underline{y})=\int \ldots \int f(\underline{x}) e^{i \operatorname{Tr}(\underline{x} \underline{y})} d x .
$$




\section{Chapter 3}

\section{Fourth Power Moment of Hecke $L$-functions over Totally Real Number Fields}

\subsection{Preliminaries}

Before proving Theorems 1.1.1 and 1.1.2, we need some further definitions and results.

Let $\mathfrak{a} \in I$ be an integral ideal, with $\mathfrak{a}=(a)$ where $a \in \mathcal{F}$. Then the Größencharacters $\lambda$ are given by

$$
\lambda_{\underline{\nu}}(a)=\left|a_{1}\right|^{i \nu_{1}} \cdots\left|a_{d}\right|^{i \nu_{d}}
$$

with

$$
\begin{aligned}
& \sum_{j=1}^{d} \nu_{j}=0, \\
& \sum_{j=1}^{d} \nu_{j} \log \left(\left|\epsilon_{j}\right|\right) \in 2 \pi \mathbb{Z},
\end{aligned}
$$

for $k=1, \ldots, d-1$, c.f. [L90, 3.2]. The conditions (3.1.2) make sure that $\lambda$ really is a character on ideals.

Using the normalization condition (3.1.1) we identify the $d$-dimensional vector $\left(\nu_{1}, \ldots, \nu_{d}\right)$ with the $(d-1)$-dimensional vector $\underline{\nu}=\left(\nu_{1}, \ldots, \nu_{d-1}\right)$ and write the character as

$$
\lambda_{\underline{\nu}}(a)=\left|\frac{a_{1}}{a_{d}}\right|^{i \nu_{1}} \cdots\left|\frac{a_{d-1}}{a_{d}}\right|^{i \nu_{d-1}} ;
$$

the condition 3.1 .2 changes to

$$
\sum_{j=1}^{d-1}\left(2 \nu_{j}+\sum_{\substack{l=1 \\ l \neq j}}^{d-1} \nu_{l}\right) \log \left(\left|\epsilon_{k}\right|\right) \in 2 \pi \mathbb{Z}
$$

for $k=1, \ldots, d-1$. We denote by $\Lambda^{d-1}$ the lattice consisting of the $(d-1)$-dimensional vectors satisfying (3.1.3). 
In the special case $d=2$ the lattice is given by $\frac{\pi}{\log \epsilon} \mathbb{Z}$ where $\epsilon$ is the fundamental unit. We denote by $\bar{\nu}=\left(\bar{\nu}_{1}, \ldots, \bar{\nu}_{d-1}\right)$ the unique basis element with

$$
\sum_{j=1}^{d-1}\left(2 \bar{\nu}_{j}+\sum_{\substack{l=1 \\ l \neq j}}^{d-1} \bar{\nu}_{l}\right) \log \left(\left.\right|_{k} \epsilon_{j} \mid\right)=2 \pi \quad \text { for all } k .
$$

In the following we identify a Größencharacter $\lambda_{\underline{\nu}}$ with its exponent $\underline{\nu}$.

At several points we interpret $\underline{\nu} \in \Lambda^{d-1}$ as a $d$-dimensional vector with $\nu_{d}=-\operatorname{Tr}(\underline{\nu})$.

We denote by $\zeta_{K}(s, \underline{\nu})$ the Hecke $L$-function given by

$$
\zeta_{K}(s, \underline{\nu})=\sum_{\substack{\mathfrak{n} \neq 0 \\ \mathfrak{n}=(n) \\ n \in \mathfrak{o}^{*}}} \lambda_{\underline{\nu}}(n)(N \mathfrak{n})^{-s}
$$

for $\underline{\nu} \in \Lambda^{d-1}$ and $\operatorname{Re} s>1$. For $\underline{\nu}=\underline{0}$, the Hecke $L$-function reduces to the Dedekind $\zeta$-function. The Hecke $L$-function $\zeta_{K}(s, \underline{\nu})$ has an analytic continuation to the whole plane, cf. [S97, page 228] and $[$ C90 page 1, equation (2)], with a simple pole at $s=1$ if $\underline{\nu}=\underline{0}$, it is an entire function if $\underline{\nu} \neq 0$.

We collect some estimates for Hecke $L$-functions which will be needed for the computation of the asymptotic formula.

On the central line the Hecke $L$-functions have the subconvexity bound

$$
\left|\zeta_{K}\left(\frac{1}{2}+i t, \underline{\nu}\right)\right| \ll(2+|t|+\operatorname{Tr}(|\underline{\nu}|))^{\frac{1}{6}+\varepsilon}
$$

see [S97, page 227].

Furthermore the inverse of the Hecke $L$-function satisfies the following estimate on $\operatorname{Re} s=1$ :

\section{(3.1.1) Lemma}

Let $\underline{\nu} \in \Lambda^{d-1}$ and $s=\sigma+i$ with $|t| \gg 1$.

Then the inverse of the Hecke L-function is bounded from above by

$$
\left|\frac{1}{\zeta_{K}(1+i t, \underline{\nu})}\right| \ll(1+|t|+\operatorname{Tr}(|\underline{\nu}|))^{\varepsilon}
$$

for all $\varepsilon>0$.

Proof.

We follow the arguments in the classical case of the Riemann $\zeta$-function, cf. [T51, §3.6].

For $\sigma>1$ we consider the expression

$$
\begin{aligned}
& \zeta_{K}(\sigma, \underline{0})^{3}\left|\zeta_{K}(\sigma+i t, \underline{\nu})\right|^{4}\left|\zeta_{K}(\sigma+2 i t, 2 \underline{\nu})\right| \\
= & \exp \left\{\sum_{m=1}^{\infty} \sum_{\mathfrak{p}=(p)} \frac{3+4 \cos \left(m \log \left|\lambda_{\underline{\nu}}(p)\right|-m t \log (N \mathfrak{p})\right)+\cos \left(2\left(m \log \left|\lambda_{\underline{\nu}}(p)\right|-m t \log (N \mathfrak{p})\right)\right)}{m(N \mathfrak{p})^{m \sigma}}\right\} .
\end{aligned}
$$

The inequality $3+4 \cos (\phi)+\cos (2 \phi)=2(1+\cos (\phi))^{2} \geq 0$ shows that every term in the above sum is non-negative. Hence $\zeta_{K}(\sigma, \underline{0})^{3}\left|\zeta_{K}(\sigma+i t, \underline{\nu})\right|^{4}\left|\zeta_{K}(\sigma+2 i t, 2 \underline{\nu})\right| \geq 1$. 
Rearranging the inequality we get

$$
\left|\frac{1}{\zeta_{K}(\sigma+i t, \underline{\nu})}\right| \leq \zeta_{K}(\sigma, \underline{0})^{\frac{3}{4}}\left|\zeta_{K}(\sigma+2 i t, 2 \underline{\nu})\right|^{\frac{1}{4}} .
$$

We bound the factors on the right hand side separately.

Duke [D89, equation (1.2.8)] gives a bound for the second term $\zeta_{K}(s, \underline{\nu})$, namely

$$
\left|\zeta_{K}(s, \lambda)\right| \ll\left[A q^{\frac{1}{d}}(1+|s|+\|\lambda\|)\right]^{d \frac{1-\sigma-\delta_{0}}{2}}
$$

for $\delta_{0} \leq \sigma \leq 1-\delta_{0}, 0>\delta_{0} \geq-\frac{1}{2}$. Here $\lambda$ is a Größencharacter modulo $\mathfrak{q}$ and $q=N \mathfrak{q}$, the constant $A$ depends on the discriminant and the degree $n$.

The first factor, $\zeta_{K}(s, \underline{0})$, has a pole at $s=1$, hence it grows like $\frac{c}{\sigma-1}$ for $\sigma \rightarrow 1$, with a constant $c$. Combining these estimates we obtain

$$
\left|\frac{1}{\zeta_{K}(\sigma+i t, \underline{\nu})}\right| \ll(1+|t|+\operatorname{Tr}(|\underline{\nu}|))^{\frac{\varepsilon}{4}}(\sigma-1)^{-\frac{3}{4}} .
$$

On the other hand, we consider

$$
\zeta_{K}(1+i t, \underline{\nu})-\zeta_{K}(\sigma+i t, \underline{\nu})=-\int_{1}^{\sigma} \zeta_{K}^{\prime}(x+i t, \underline{\nu}) d x .
$$

To estimate the derivative $\zeta_{K}^{\prime}(s, \underline{\nu})$ we again use [D89, equation (1.2.8)] and then apply Cauchy's formula for a circle with radius $(1+|t|+\operatorname{Tr}(|\underline{\nu}|))^{-\varepsilon}$ to obtain

$$
\left|\zeta_{K}^{\prime}(s, \underline{\nu})\right| \ll(1+|t|+\operatorname{Tr}(|\underline{\nu}|))^{2 \varepsilon} .
$$

Inserting this into the above equation we get the bound

$$
\zeta_{K}(1+i t, \underline{\nu})-\zeta_{K}(\sigma+i t, \underline{\nu}) \ll(\sigma-1)(1+|t|+\operatorname{Tr}(|\underline{\nu}|))^{2 \varepsilon} .
$$

Combining the estimates (3.1.7) and (3.1.8) leads to the lower bound

$$
\left|\zeta_{K}(1+i t, \underline{\nu})\right|>A_{1}(1+|t|+\operatorname{Tr}(|\nu|))^{-\frac{\varepsilon}{4}}(\sigma-1)^{\frac{3}{4}}-A_{2}(\sigma-1)(1+\operatorname{Tr}(|\nu|)+|t|)^{2 \varepsilon} .
$$

The two terms on the right have the same order of magnitude for $\sigma-1 \asymp(1+|t|+\operatorname{Tr}(|\nu|))^{-9 \varepsilon}$, e. g. choose $\sigma-1=\left(\frac{A_{1}}{2 A_{2}}\right)^{4}(1+|t|+\operatorname{Tr}(|\nu|))^{-9 \varepsilon}$. Hence, with some $A_{3}>0$ small enough, we obtain

$$
\left|\zeta_{K}(1+i t, \underline{\nu})\right|>A_{3}(1+|t|+\operatorname{Tr}(|\nu|))^{-\varepsilon}
$$

and the statement follows.

Let $\xi \in \mathbb{C}, \underline{\nu} \in \Lambda^{d-1}, \mu \in \mathbb{N}_{0}$ and $\mathfrak{n}=(n)$ with $n \succ 0$. We define

$$
\sigma_{\xi}(n, \underline{\nu})=\sum_{\substack{\mathfrak{d} \mid \mathfrak{n} \\ \mathfrak{d}=(d)}} \lambda_{\underline{\nu}}(d)(N \mathfrak{d})^{\xi}
$$

where the sums run over $d \succ 0$, c.f. [BM01, (3.15)]. For $\underline{\nu}=\underline{0}$ we write $\sigma_{\xi}(n, \underline{0})=\sigma_{\xi}(n)$. 
For $\operatorname{Re} s, t>1$ the Hecke $L$-functions and the divisor functions $\sigma_{\xi}(n, \underline{\nu})$ are related via the equality

$$
\sum_{m \in \mathcal{F}} \lambda_{\underline{\nu}}(m) N(m)^{-s} \sigma_{s-t}(m, \underline{\mu}-\underline{\nu})=\zeta_{K}(s, \underline{\nu}) \zeta_{K}(t, \underline{\mu})
$$

In our case of a totally real number field there are infinitely many generators for each ideal, namely $(x)=\left(\prod_{j=1}^{d-1}{ }_{j} \epsilon^{\nu_{j}} x\right)$ for every $\underline{\nu} \in \mathbb{Z}^{d-1}$. To overcome this difficulty we introduce a partition of unity, see [BM01, Lemma 2.1, (2.3)ff].

Let $\mathrm{p}$ be such that its Fourier transform $\widehat{\mathrm{p}}(\underline{y})$ is even, real-valued, smooth, supported in $\left(-\frac{\pi}{2}, \frac{\pi}{2}\right)$ in each variable $y_{j}, j=1, \ldots, d-1$, and with $\widehat{\mathrm{p}}(\underline{0})=1$. Then the function $\mathrm{p}$ is even, real-valued, smooth and of rapid decay on $\mathbb{R}^{d-1}$, and for $\underline{x}, \underline{y} \in \mathbb{R}^{d-1}$ we have

$$
\begin{aligned}
& \sum_{\underline{n} \in \mathbb{Z}^{d-1}} \mathrm{p}(\underline{x}+\underline{n})=1, \\
& \sum_{\underline{n} \in \mathbb{Z}^{d-1}} \mathrm{p}(\underline{x}+\underline{n}) \mathrm{p}(\underline{y}+\underline{n})=\frac{1}{(2 \pi)^{d-1}} \int_{\mathbb{R}^{d-1}} \ldots \int \widehat{\mathrm{p}}(\underline{\xi})^{2} e^{i \operatorname{Tr}((\underline{y}-\underline{x}) \underline{\xi})} d \underline{\xi} .
\end{aligned}
$$

This follows by Poisson's summation formula and by Parseval's formula.

We define

$$
\Psi(x)=\frac{1}{2^{d-1}} \mathrm{p}\left(\log \left|\frac{x_{1}}{x_{d}}\right| \bar{\nu}_{1}, \ldots, \log \left|\frac{x_{d-1}}{x_{d}}\right| \bar{\nu}_{d-1}\right)
$$

for an element $x \in K \backslash\{0\}$, with $x=\left(x_{1}, \ldots, x_{d}\right)$ as a vector in $\mathbb{R}^{d}$, and $\bar{\nu}$ the basis element of $\Lambda^{d-1}$ chosen in 3.1.4. Further let

$$
\Psi_{+}(x)= \begin{cases}2^{d-1} \Psi(x), & x \succ 0, \\ 0, & \text { else. }\end{cases}
$$

Then for $x \in K \backslash\{0\}$ the identities (3.1.11) and 3.1.12 imply

$$
\begin{aligned}
& \sum_{\epsilon} \Psi(\varepsilon x)=1, \\
& \sum_{\epsilon} \Psi(\varepsilon x)^{2}=\frac{1}{(4 \pi)^{d-1}} \int_{\mathbb{R}^{d-1}} \ldots \int \widehat{\mathrm{p}}^{2}(\underline{\xi}) d \underline{\xi}=: c_{\Psi} .
\end{aligned}
$$

For $\Psi_{+}$, with $x \succ 0$ we have the similar results

$$
\begin{aligned}
& \sum_{\epsilon \succ 0} \Psi_{+}(\varepsilon x)=1, \\
& \sum_{\epsilon \succ 0} \Psi_{+}(\varepsilon x)^{2}=2^{1-d} c_{\Psi} .
\end{aligned}
$$

Thus for any function $f$ defined over the positive reals, we have

$$
f(N \mathfrak{a})=\sum_{\substack{a \in \mathfrak{o}^{*} \\(a)=\mathfrak{a}}} f(|N(a)|) \Psi_{+}(a) .
$$




\subsubsection{Special Functions}

For the spectral decomposition we shall need the Eisenstein series and Casimir elements of the Lie group $G=\mathrm{PSL}_{2}(\mathbb{R})^{d}$, see BM01, Section 3].

The Hilbert modular group $\Gamma=\mathrm{PSl}_{2}(\mathfrak{o})$ of $K$ is a discrete subgroup of the Lie group $G$, via the embedding $g \mapsto\left(g_{1}, \ldots, g_{d}\right)$ of $\mathrm{PSL}_{2}(\mathfrak{o})$ into $G$ where the conjugation is applied elementwise. The group $G$ has the Iwasawa decomposition $G=$ NAK where

$$
\begin{array}{cc}
\mathrm{N}=\left\{\mathrm{n}[\underline{x}] \mid \underline{x} \in \mathbb{R}^{d}\right\}, & \mathrm{n}[\underline{x}]=\left(\left[\begin{array}{cc}
1 & x_{j} \\
& 1
\end{array}\right]\right)_{j=1, \ldots, d}, \\
\mathrm{~A}=\left\{\underline{\mathrm{a}}[\underline{y}] \mid \underline{y} \in(0, \infty)^{d}\right\}, & \mathrm{a}[\underline{y}]=\left(\left[\begin{array}{cc}
\sqrt{y_{j}} & \\
1 / \sqrt{y_{j}}
\end{array}\right]\right)_{j=1, \ldots, d}, \\
\mathrm{~K}=\left\{\mathrm{k}[\underline{\theta}] \mid \underline{\theta} \in(\mathbb{R} / \pi \mathbb{Z})^{d}\right\}, & \mathrm{k}[\underline{\theta}]=\left(\left[\begin{array}{cc}
\cos \left(\theta_{j}\right) & \sin \left(\theta_{j}\right) \\
-\sin \left(\theta_{j}\right) & \cos \left(\theta_{j}\right)
\end{array}\right]\right)_{j=1, \ldots, d}
\end{array}
$$

Every $\mathrm{g} \in G$ equals a product $\mathrm{n}[\underline{x}] \mathrm{a}[\underline{y}] \mathrm{k}[\underline{\theta}]$. In the following we use this decomposition of $G$ without mention. If a factor of the product contains expressions of $K$, this should be understood as resulting from the embedding.

A matrix that will come up several times is $\mathrm{w}$, defined as

$$
\mathrm{w}=\mathrm{k}\left[\frac{\pi}{2}\right]=\left(\left[-1{ }^{1}\right]\right)_{j=1, \ldots, d} .
$$

The Lie algebra $\mathfrak{g}$ of $G$ has the basis

$$
\begin{array}{rlrl}
\mathbf{w}_{j} & =\frac{1}{2} \partial_{\theta_{j}}, \\
\mathbf{e}_{j}^{+} & =e^{2 i \theta_{j}}\left(i y_{j} \partial_{x_{j}}+y_{j} \partial_{y_{j}}-\frac{1}{2} i \partial_{\theta_{j}}\right), & \\
\mathbf{e}_{j}^{-} & =\overline{\mathbf{e}_{j}^{+}}, & j=1, \ldots, d .
\end{array}
$$

The basis elements satisfy the relations

$$
\begin{aligned}
{\left[\mathbf{w}_{j}, \mathbf{e}_{j}^{ \pm}\right] } & = \pm i \mathbf{e}_{j}^{ \pm}, \\
{\left[\mathbf{e}_{j}^{+}, \mathbf{e}_{j}^{-}\right] } & =-2 i \mathbf{w}_{j}, \quad j=1, \ldots, d,
\end{aligned}
$$

and $\left[\mathbf{x}_{j_{1}}, \mathbf{x}_{j_{2}}\right]=0$ for $j_{1} \neq j_{2}$ and $\mathbf{x}_{j_{i}} \in\left\{\mathbf{w}_{j_{i}}, \mathbf{e}_{j_{i}}^{+}, \mathbf{e}_{j_{i}}^{-}\right\}$. Thus the center of the universal enveloping algebra $\mathcal{U}$ of $\mathfrak{g}$ is the polynomial ring in the $d$ Casimir elements

$$
\Omega_{j}=-\mathbf{e}_{j}^{+} \mathbf{e}_{j}^{-}+\mathbf{w}_{j}^{2}-i \mathbf{w}_{j}=-y_{j}^{2}\left(\partial_{x_{j}}^{2}+\partial_{y_{j}}^{2}\right)+y_{j} \partial_{x_{j}} \partial_{\theta_{j}}
$$

with $j=1, \ldots, d$.

We say that a function $f$ on $G$ is left $\Gamma$-automorphic of weight $2 q=2\left(q_{1}, \ldots, q_{d}\right)$ with $q \in \mathbb{Z}^{d}$ if for any $\mathrm{g} \in G$ it holds true that

$$
f(\gamma \mathrm{g})=f(\mathrm{~g}), \text { for } \gamma \in \Gamma ; \quad f(\operatorname{gk}[\underline{\theta}])=e^{2 i \operatorname{Tr}(q \underline{\theta})} f(\mathrm{~g}) .
$$

The second one is equivalent to

$$
\mathbf{w}_{j} f=i q_{j} f, \quad j=1, \ldots, d .
$$


Further

$$
\mathbf{e}_{j}^{ \pm} f \text { are } \Gamma \text {-automorphic of weight } 2\left(q \pm 1_{j}\right)
$$

where $1_{j}$ is the vector with zeros everywhere except a one on the $j$-th place. Such an $f$ trivially satisfies $f(\mathrm{n}[n] \mathrm{g})=f(\mathrm{~g})$ for all $n \in \mathfrak{o}$. Thus, under a suitable smoothness condition, $f$ has a Fourier expansion in terms of the additive characters given by

$$
\psi_{n}(\mathrm{~g})=\exp (2 \pi i \operatorname{Tr}(n \underline{x})), \quad n \in \mathfrak{o}
$$

where $\underline{x}$ comes from the Iwasawa decomposition of $g$.

\section{Eisenstein Series}

Now we introduce the Eisenstein series. Let $\Gamma_{\infty}=\Gamma \cap \mathrm{N}$ be the stabilizer in $\Gamma$ of the point at infinity, and let $q=\left(q_{1}, \ldots, q_{d}\right) \in \mathbb{Z}^{d}, \underline{\nu} \in \Lambda^{d-1}$ and $\mathrm{g} \in G$. Then for Re $s>\frac{1}{2}$ we define the Eisenstein series

$$
\begin{aligned}
E_{q}(\mathrm{~g} ; s, \underline{\nu}) & :=\sum_{\gamma \in \Gamma_{\infty} \backslash \Gamma} \phi_{q}\left(\gamma \mathrm{g} ; s+i \nu_{1}, \ldots, s+i \nu_{d-1}, s-i \operatorname{Tr}(\underline{\nu})\right), \\
\text { where } \quad \phi_{q}(\mathrm{~g} ; \underline{s}) & =\prod_{j=1}^{d} y_{j}^{s_{j}+\frac{1}{2}} e^{2 i \operatorname{Tr}(q \underline{\theta})}
\end{aligned}
$$

where $\underline{y}$ and $\underline{\theta}$ come from the Iwasawa decomposition of $\mathrm{g}$.

As a function of $s$ the Eisenstein series has a meromorphic continuation to $\mathbb{C}$ and satisfies a functional equation:

\section{(3.1.2) Lemma}

Let $q=\left(q_{1}, \ldots, q_{d}\right) \in \mathbb{Z}^{d}, \underline{\nu} \in \Lambda^{d-1}$ and $\nu_{d}=-\operatorname{Tr}(\underline{\nu})=-\sum_{j=1}^{d-1} \nu_{j}$. Then, as a function of $s$, the Eisenstein series $E_{q}(\mathrm{~g} ; s, \underline{\nu})$ continues meromorphically to $\mathbb{C}$, and satisfies the functional equation

$$
E_{q}(\mathrm{~g} ;-s,-\underline{\nu})=\pi^{-2 d s} \prod_{j=1}^{d}\left[\frac{\Gamma\left(\frac{1}{2}+s+i \nu_{j}+\left|q_{j}\right|\right)}{\Gamma\left(\frac{1}{2}-s-i \nu_{j}+\left|q_{j}\right|\right)}\right] \frac{\zeta_{K}(1+2 s,-2 \underline{\nu})}{\zeta_{K}(1-2 s, 2 \underline{\nu})} E_{q}(\mathrm{~g} ; s, \underline{\nu}) .
$$

If $E_{q}(\mathrm{~g} ; s, \underline{\nu})$ is holomorphic, it is a $\Gamma$-automorphic function of weight $2 q$.

If $\operatorname{Re} s>0$, a singularity occurs only for $\underline{\nu}=\underline{0}$ and $q=\underline{0}$ and $E_{\underline{0}}(\mathrm{~g} ; s, \underline{0})$ has a simple pole at $s=\frac{1}{2}$. Furthermore $E_{q}(\mathrm{~g} ; s, \underline{\nu})$ has the Fourier expansion

$$
\begin{aligned}
& e^{-2 i \operatorname{Tr}(q \underline{\theta})} E_{q}(\mathrm{~g} ; s, \underline{\nu})=(N \underline{y})^{\frac{1}{2}+s} \lambda_{\underline{\nu}}(\underline{y}) \\
& +\pi^{\frac{d}{2}}(-1)^{\operatorname{Tr}(q)}(N \underline{y})^{\frac{1}{2}-s} \lambda_{-\underline{\nu}}(\underline{y}) \prod_{j=1}^{d}\left[\frac{\Gamma\left(s+i \nu_{j}\right)}{\Gamma\left(\frac{1}{2}+s+i \nu_{j}\right)} \prod_{k=1}^{\left|q_{j}\right|-1}\left(\frac{s+i \nu_{j}-k-\frac{1}{2}}{\frac{1}{2}+s+i \nu_{j}+k}\right)\right] \frac{\zeta_{K}(2 s,-2 \underline{\nu})}{\zeta_{K}(1+2 s,-2 \underline{\nu})} \\
& +\pi^{\frac{d}{2}}(-1)^{\operatorname{Tr}(q)} \frac{\pi^{d s}}{\zeta_{K}(1+2 s,-2 \underline{\nu})} \sum_{n \in \mathfrak{o}_{*}} \frac{\lambda_{\underline{\nu}}(n)}{|N n|^{s+\frac{1}{2}}} \sigma_{2 s}(n, 2 \underline{\nu}) \psi_{n}(\mathrm{n}[\underline{x}]) \prod_{j=1}^{d} \frac{W_{q_{j} \operatorname{sgn}\left(n_{j}\right), s+i \nu_{j}}\left(4 \pi\left|n_{j}\right| y_{j}\right)}{\Gamma\left(\frac{1}{2}+s+i \nu_{j}+q_{j} \operatorname{sgn}\left(n_{j}\right)\right)}
\end{aligned}
$$

where $\mathrm{g}=\mathrm{n}[\underline{x}] \mathrm{a}[\underline{y}] \mathrm{k}[\underline{\theta}]$, and $W_{a, b}$ is the Whittaker function, see Section 5.1 . The sum over $n \in \mathfrak{o}^{*}$ converges absolutely and uniformly for all parameters involved. For $N \underline{y} \rightarrow \infty$ it is of exponential decay. 
Proof.

We follow the proof of [BM01, Lemma 3.1].

The functional equation follows from the Fourier expansion. Thus it is enough to prove the Fourier expansion. The Bruhat decomposition implies

$$
E_{q}(\mathrm{~g} ; s, \underline{\nu})=\phi_{q}(\mathrm{~g} ; \underline{s})+\sum_{\substack{\mathfrak{c}=(c) \\ c \succ 0}} \sum_{\substack{\mathfrak{a}=(a),(a, c)=1 \\ \bmod (c)}} \sum_{n \in \mathfrak{o}} \phi_{q}\left(\mathrm{a}\left[\frac{1}{c^{2}}\right] \mathrm{wn}\left[\frac{a}{c}+n\right] \mathrm{g} ; \underline{s}\right)
$$

where the vector $\underline{s}=\left(s+i \nu_{1}, \ldots, s+i \nu_{d-1}, s-i \operatorname{Tr}(\underline{\nu})\right)$, and the matrix $\mathrm{w}=\mathrm{k}\left[\frac{\pi}{2}\right]$ is defined in 3.1.16). Now, applying Poisson's formula to the sum over $n$ yields

$$
\sum_{n \in \mathfrak{o}} \phi_{q}\left(\mathrm{a}\left[\frac{1}{c^{2}}\right] \operatorname{wn}\left[\frac{a}{c}+n\right] \mathrm{g} ; \underline{s}\right)=\sum_{n \in \mathfrak{o}^{*}} e\left[\frac{a n}{c}\right] \int_{\mathrm{N}} \psi_{n}^{-1}(\mathrm{n}) \phi_{q}\left(\mathrm{a}\left[\frac{1}{c^{2}}\right] \mathrm{wng} ; \underline{s}\right) d \mathrm{n} .
$$

Then, using the equality a $\left[\frac{1}{c^{2}}\right] \operatorname{wn}[x] \mathrm{a}[y]=\mathrm{wn}\left[c^{2} x\right] \mathrm{a}\left[c^{2} \underline{y}\right]$ and the coordinates of the Iwasawa decomposition of $\mathrm{g}$, the right hand side changes to

$$
\frac{e^{2 i \operatorname{Tr}(q \underline{\theta})}}{(N c)^{2}} \sum_{n \in \mathfrak{o}^{*}} e\left[\frac{a n}{c}\right] \psi_{n}(\mathrm{n}[\underline{x}]) \mathcal{A}_{\frac{n}{c^{2}}} \phi_{q}\left(\mathrm{a}\left[c^{2} \underline{y}\right] ; \underline{s}\right)
$$

where $\mathcal{A}_{n}$ is the Jacquet operator given by

$$
\mathcal{A}_{n} f(\mathrm{~g})=\int_{N} \psi_{n}^{-1}(\mathrm{n}) f(\mathrm{wng}) d \mathrm{n}
$$

An easy computation shows

$$
\mathrm{wn}[x] \mathrm{a}[y]=\left(\begin{array}{cc}
1 & \frac{-x}{|x+i y|^{2}} \\
0 & 1
\end{array}\right)\left(\begin{array}{cc}
\frac{\sqrt{y}}{|x+i y|} & 0 \\
0 & \frac{|x+i y|}{\sqrt{y}}
\end{array}\right)\left(\begin{array}{cc}
\frac{-x}{|x+i y|} & \frac{y}{|x+i y|} \\
\frac{-y}{|x+i y|} & \frac{-x}{|x+i y|}
\end{array}\right)=\mathrm{n}\left[\frac{-x}{|x+i y|^{2}}\right] \mathrm{a}\left[\frac{y}{|x+i y|^{2}}\right] \mathrm{k}\left[i \log \left(\frac{x+i y}{|x+i y|}\right)\right] .
$$

Thus, using the definition of $\phi_{q}$ and inserting these coordinates, the Jacquet operator in 3.1.25 can be written as

$$
\begin{aligned}
\mathcal{A}_{n} \phi_{q}(\mathrm{a}[\underline{y}] ; \underline{s}) & =\prod_{j=1}^{d} \int_{\mathbb{R}} \exp \left(-2 \pi i x_{j} n_{j}\right)\left(\frac{y_{j}}{\left|x_{j}+i y_{j}\right|^{2}}\right)^{\frac{1}{2}+s+i \nu_{j}}\left(\frac{x_{j}+i y_{j}}{\left|x_{j}+i y_{j}\right|}\right)^{-2 q_{j}} d x_{j} \\
& =(N \underline{y})^{\frac{1}{2}-s} \lambda_{\underline{\nu}}(\underline{y}) \prod_{j=1}^{d} \int_{\mathbb{R}} \frac{\exp \left(2 \pi i n_{j} y_{j} \xi_{j}\right)}{\left(1+\xi_{j}^{2}\right)^{\frac{1}{2}+s-i \nu_{j}}}\left(\frac{i+\xi_{j}}{\left|i+\xi_{j}\right|}\right)^{2 q_{j}} d \xi_{j} .
\end{aligned}
$$

The last integral is known, see [BM01, (3.22)], and for Re $s>0$ and $u \in \mathbb{R}$ satisfies the equality

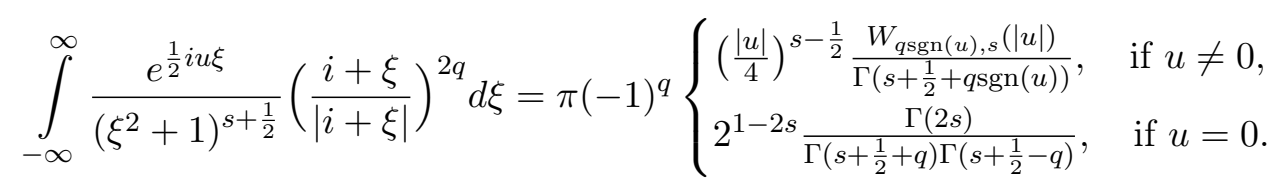


Inserting this into (3.1.24), for the second term of the right hand side we obtain

$$
\begin{aligned}
e^{2 i \operatorname{Tr}(q \theta)}(-1)^{\operatorname{Tr}(q)} \pi^{d} & \sum_{\substack{\mathfrak{c}=(c) \\
c \succ 0}} \frac{\lambda_{-2 \underline{\nu}}(c)}{(N c)^{1+2 s}} \sum_{\substack{\mathfrak{a} \bmod c \\
\mathfrak{a}=(a),(a, c)=1}} e\left[\frac{a n}{c}\right] \times \\
& \times\left\{2^{d(1-2 s)}(N \underline{y})^{\frac{1}{2}-s} \lambda_{\underline{\nu}}(\underline{y}) \prod_{j=1}^{d} \frac{\Gamma\left(2 s+2 i \nu_{j}\right)}{\Gamma\left(s+i \nu_{j}+\frac{1}{2}+q_{j}\right) \Gamma\left(s+i \nu_{j}+\frac{1}{2}-q_{j}\right)}\right. \\
& \left.+\pi^{d s-\frac{d}{2}} \sum_{n \in \mathfrak{o}^{*}} \frac{\lambda_{\underline{\nu}}(n)}{(N n)^{-s+\frac{1}{2}}} \psi_{n}(\mathrm{n}[\underline{x}]) \prod_{j=1}^{d} \frac{W_{q_{j} \operatorname{sgn}\left(n_{j}\right), s+i \nu_{j}\left(4 \pi\left|n_{j}\right| y_{j}\right)}}{\Gamma\left(s+i \nu_{j}+\frac{1}{2}+q_{j} \operatorname{sgn}\left(n_{j}\right)\right)}\right\} .
\end{aligned}
$$

Finally we need the Ramanujan expansion with a twist of Größencharacters, namely

$$
\sum_{\mathfrak{c}=(c)} \frac{\lambda_{2 \underline{\nu}}(c)}{(N c)^{s}} \sum_{\substack{\mathfrak{a} \bmod c \\ \mathfrak{a}=(a),(a, c)=1}} e\left[\frac{a n}{c}\right]=\frac{1}{\zeta_{K}(s, 2 \underline{\nu})} \begin{cases}\zeta_{K}(s-1,2 \underline{\nu}), & \text { if } n=0, \\ \sigma_{1-s}(n, 2 \underline{\nu}), & \text { if } n \neq 0\end{cases}
$$

to reformulate the sum over $c$ and $a$. Then an application of the duplication formula for the $\Gamma$-functions if $n=0$, leads to the Fourier expansion.

To see the convergence, we shift the contours of the Jacquet operator appropriately, and see that

$$
\mathcal{A}_{\frac{n}{c^{2}}} \phi_{q}\left(\mathrm{a}\left[c^{2} \underline{y}\right] ; s\right) \ll_{K}(N c \sqrt{N \underline{y}})^{1-2 \operatorname{Re} s} \exp (-a \operatorname{Tr}(|n \underline{y}|)),
$$

for $\operatorname{Re} s>0$, and $a>0$. Then, for $\operatorname{Re} s>0$ the sum over $n$ is bounded by

$$
\ll\left((N c)^{2} N \underline{y}\right)^{\frac{1}{2}-\operatorname{Re} s} \sum_{n \in \mathcal{F}} K_{0}\left(a(N(n \underline{y}))^{\frac{1}{2}}\right),
$$

where $K_{0}$ is the $K$-Bessel function of order 0 , see Section 5.2 .

Hence, (3.1.24 converges absolutely for $\operatorname{Re} s>\frac{1}{2}$, and the last assertion of the lemma follows.

\subsubsection{Eigenvalues and Hecke Operators}

In this section we follow the steps of [BM01, Section 4] to obtain a geometric sum formula for the Hilbert modular group $\Gamma$.

Recall that $G=\mathrm{PSL}_{2}(\mathbb{R})^{d}$. Let $L^{2}(\Gamma \backslash G)$ be the Hilbert space consisting of all left $\Gamma$-automorphic functions on $G$ which are square integrable against the measure $d g$. Let ${ }^{0} L^{2}(\Gamma \backslash G)$ be its cuspidal subspace. Then we have the decomposition

$$
\begin{aligned}
L^{2}(\Gamma \backslash G) & =\mathbb{C} \oplus{ }^{0} L^{2} \oplus \mathcal{E}, \\
{ }^{0} L^{2}(\Gamma \backslash G) & =\overline{\bigoplus V}=\overline{\bigoplus \bigoplus_{q \in \mathbb{Z}^{d}} V_{q}}, \\
\mathcal{E} & =\overline{\bigoplus_{\underline{\nu} \in \Lambda^{d-1}} \bigoplus_{q \in \mathbb{Z}^{d}} \mathcal{E}_{\underline{\nu}, q}}
\end{aligned}
$$

where $V$ runs over an orthogonal system of right irreducible cuspidal subspaces and decomposes into $\bigoplus V_{q}$. In every subspace $V_{q}$ the matrices $\mathrm{k}[\underline{\theta}]$, defined in Section 3.1.1, act as the multiplication by $e^{2 i \operatorname{Tr}(q \underline{\theta})}$, and $\operatorname{dim} V_{q} \leq 1$. 
The subspace $\mathcal{E}_{\underline{\nu}, q}$ is generated by the Eisenstein series $E_{q}(\mathrm{~g} ; s, \underline{\nu})$, defined in 3.1 .22 , via

$$
\mathcal{E}_{\underline{\nu}, q}=\left\{\left.\int_{-\infty}^{\infty} u(t) E_{q}\left(\mathrm{~g} ; \frac{1}{2}+i t, \underline{\nu}\right) d t\left|\int_{-\infty}^{\infty}\right| u(t)\right|^{2} d t<\infty\right\} .
$$

The Casimir operator $\Omega_{j}$ defined in 3.1 .19 acts on $V$ as multiplication by a constant, namely

$$
\left.\Omega_{j}\right|_{V}=\left(\frac{1}{4}+\kappa_{j}^{2}\right) \cdot 1
$$

with $\kappa_{j} \in \mathbb{C}$. Further we note that

$$
\left.\mathbf{w}_{j}\right|_{V_{q}}=i q_{j} \cdot 1,\left.\quad \mathbf{w}_{j}\right|_{\varepsilon_{\underline{\nu}, q}}=i q_{j} \cdot 1, \quad j=1, \ldots, d .
$$

Now we restrict our attention to the cuspidal subspaces $V$ and deduce the possible values of $\kappa_{j}$. We observe that the basis elements $\mathbf{e}_{j}^{ \pm}$of $\mathfrak{g}$ act on $V$ and 3.1 .20 implies

$$
\mathbf{e}_{j}^{ \pm}: V_{q} \rightarrow V_{q \pm 1_{j}}
$$

where $1_{j}$ is as in 3.1 .20 .

We choose a generator $\varphi$ of $V_{q}$. Then $\varphi$ is a cusp form over $\Gamma \backslash G$, i.e. it is an element of ${ }^{0} L^{2}(\Gamma \backslash G)$, and it is an eigenfunction of the Casimir operator $\Omega_{j}$ and the basis element $\mathbf{w}_{j}$. By the definition of the Casimir operator $\mathbf{e}_{j}^{\mp} \mathbf{e}_{j}^{ \pm}=-\Omega_{j}+\mathbf{w}_{j}^{2} \pm i \mathbf{w}_{j}$, we have

$$
\mathbf{e}_{j}^{\mp} \mathbf{e}_{j}^{ \pm} \varphi=-\left(\left( \pm q_{j}+\frac{1}{2}\right)^{2}+\kappa_{j}^{2}\right) \varphi .
$$

Then integration by parts yields

$$
\left\|\mathbf{e}_{j}^{ \pm} \varphi\right\|^{2}=-\left\langle\mathbf{e}_{j}^{\mp} \mathbf{e}_{j}^{ \pm} \varphi, \varphi\right\rangle=\left(\left( \pm q_{j}+\frac{1}{2}\right)^{2}+\kappa_{j}^{2}\right)\|\varphi\|^{2} .
$$

In particular the term $\left( \pm q_{j}+\frac{1}{2}\right)^{2}+\kappa_{j}^{2}$ is non-negative and we can choose $\kappa_{j}$ to be

$$
\text { either } \kappa_{j} \geq 0 \text { or } 0 \leq i \kappa_{j} \leq|| q_{j}\left|-\frac{1}{2}\right| \text {. }
$$

Thus, the mappings 3.1 .32 are bijective in many cases. Exceptions can occur only if $i \kappa_{j}=l_{j}-\frac{1}{2}$ with some $l_{j} \in \mathbb{N}$. More precisely

$$
V_{q} \neq\{0\} \text { and } \mathbf{e}_{j}^{ \pm} V_{q}=\{0\} \Leftrightarrow q_{j}=\mp l_{j} \text { or } l_{j}=1, q_{j}=0 .
$$

Thus the only possible values that $i \kappa_{j}$ can attain if $i \kappa_{j} \geq \frac{1}{2}$ are $l_{j}-\frac{1}{2}$ with $l_{j} \in \mathbb{N}$.

We summarize the possible cases:

(I) $q_{j} \in \mathbb{Z}, \kappa_{j} \geq 0$ (unitary principal series)

(II) $q_{j} \in \mathbb{Z}, 0 \leq i \kappa_{j}<\frac{1}{2}$ (complementary series)

(III) $q_{j} \geq l_{j}, i \kappa_{j}=l_{j}-\frac{1}{2}$ (holomorphic discrete series)

(IV) $q_{j} \leq-l_{j}, i \kappa_{j}=l_{j}-\frac{1}{2}$ (anti-holomorphic discrete series)

(V) $q_{j}=0, i \kappa_{j}=\frac{1}{2}$ (trivial representation). 
The last case (V) cannot occur, as the elements of $V$ are cusp forms.

Thus, we have the following lemma.

\section{(3.1.3) Lemma}

Let $V=\overline{\bigoplus_{q \in \mathbb{Z}^{d}} V_{q}}$ be an irreducible cuspidal subspace of ${ }^{0} L^{2}(\Gamma \backslash G)$, and let $\kappa_{j}$ be given by 3.1.31 and 3.1.33. Then for $j=1, \ldots, d$ either

$$
\text { 1. } \kappa_{j} \geq 0 \text { or } 0 \leq i \kappa_{j}<\frac{1}{2} \text {, }
$$

or 2. $i \kappa_{j}=l_{j}-\frac{1}{2}$, with $\eta_{j} q_{j} \geq l_{j}$ and $l_{j} \in \mathbb{N}$.

Here $\eta_{j}= \pm 1$, and $q_{j} \in \mathbb{Z}$.

We may choose a cusp form $\varphi_{V} \in V$ of weight $2 q_{V}$ with $q_{V}=\left(q_{1}, \ldots, q_{d}\right)$ where

$$
q_{j}= \begin{cases}0, & \text { if } 1 . \text { holds }, \\ \eta_{j} l_{j}, & \text { if 2. holds }\end{cases}
$$

such that $V=\overline{\mathcal{U} \cdot \varphi_{V}}$, with the universal enveloping algebra $\mathcal{U}$ of $\mathfrak{g}$.

The exceptional eigenvalues, i.e. the numbers $\frac{1}{4}+\kappa_{j}^{2}$ with $0 \leq i \kappa_{j}<\frac{1}{2}$, satisfy non-trivial lower bounds. The best known result is

$$
i \kappa_{j} \leq \frac{7}{64}
$$

see [BB11.

As a next step we consider the Fourier expansion of a cusp form and obtain a relation between the Fourier coefficients and the Hecke eigenvalues.

Let $\varphi_{V} \in V$ be a cusp form of weight $2 q_{V}$. Its Fourier expansion is given by

$$
\varphi_{V}(\mathrm{~g})=(-1)^{\operatorname{Tr}\left(q_{V}\right)} e^{2 i \operatorname{Tr}\left(q_{V} \underline{\theta}\right)} \sum_{n \in \mathfrak{o}^{*}} \frac{\varrho_{V}(n)}{\sqrt{|N n|}} \psi_{n}(\mathrm{~g}) \prod_{j=1}^{d} \frac{W_{q_{j} \operatorname{sgn}\left(n_{j}\right), i \kappa_{j}}\left(4 \pi\left|n_{j}\right| y_{j}\right)}{\Gamma\left(\frac{1}{2}+i \kappa_{j}+q_{j} \operatorname{sgn}\left(n_{j}\right)\right)}
$$

where $W_{a, b}$ is the Whittaker function given in Section 5.1. The additive character $\psi_{n}(\mathrm{~g})$ is defined in 3.1.21); the $\varrho_{V}(n)$ are certain complex numbers. This is a specialisation $\varphi=\varphi_{V}$ where $\varphi$ is a solution of the differential equation $\Omega_{j} \varphi=\left(\frac{1}{4}+\kappa_{j}^{2}\right) \varphi$ with $\varphi \in V_{q}$.

The $\Gamma$-factors do not produce zeros with the possible combinations of $q_{V}$ and $\kappa_{V}$. From now on we assume $\left\{\varphi_{V} \mid V\right\}$ to be an orthonormal system in $L^{2}(\Gamma \backslash G)$. The $\varrho_{V}(n)$ are called the Fourier coefficients of $V$. We note that the vector $\left(\varrho_{V}(n)\right)_{n \in \mathfrak{o}^{*}}$ is well-defined and stable under multiplication of $n$ with $\varepsilon={ }_{1} \epsilon^{2 \nu_{1}} \cdots_{d-1} \epsilon^{2 \nu_{d-1}}$, i. e. $\varrho_{V}(\varepsilon n)=\varrho_{V}(n)-$ a consequence of $\varphi_{V}(\mathrm{a}[\varepsilon] \mathrm{g})=\varphi_{V}(\mathrm{~g})$.

Now we consider the Hecke operators on the space $L^{2}(\Gamma \backslash G)$ given by

$$
T_{\mathfrak{n}} f(\mathrm{~g})=\frac{1}{\sqrt{N \mathfrak{n}}} \sum_{\substack{\mathfrak{d} \mid \mathfrak{n} \\ \mathfrak{d}=(d), d \succ 0}} \sum_{b \bmod d} f\left(\mathrm{n}\left[\frac{b}{d}\right] \mathrm{a}\left[\frac{n}{d^{2}}\right] \mathrm{g}\right) \quad \text { for } \mathfrak{n}=(n) \text { with } n \succ 0 .
$$

The Hecke operators preserve the orthogonal decomposition (3.1.28) and it can be shown that the equality $T_{\mathfrak{n}} T_{\mathfrak{m}}=T_{\mathfrak{m}} T_{\mathfrak{n}}$ holds for any $\mathfrak{m}, \mathfrak{n}$, and each $T_{\mathfrak{n}}$ is symmetric over $L^{2}(\Gamma \backslash G)$. Therefore, we may 
assume that $V$ is such that

$$
\left.T_{\mathfrak{n}}\right|_{V}=t_{V}((n)) \cdot 1, t_{V}((n)) \in \mathbb{R}
$$

Computing the Fourier coefficients of $T_{\mathfrak{n}} \varphi_{V}$ we have for $\mathfrak{n}=(n)$ and any $m \in \mathfrak{o}^{*}$

$$
\varrho_{V}(m) t_{V}(\mathfrak{n})=\sum_{(d) \mid(m, n)} \varrho_{V}\left(\frac{m n}{d^{2}}\right)
$$

Hence, for $m=\varepsilon \in \mathfrak{o}^{\times}$a unit and $n \succ 0$ we have

$$
\varrho_{V}(\varepsilon n)=\varrho_{V}(\varepsilon) t_{V}((n))
$$

In other words, for any $n \in \mathfrak{o}^{*}$

$$
\varrho_{V}(n)=t_{V}((n)) \cdot \begin{cases}\varrho_{V}(1), & n \succ 0, \\ \vdots & \\ \varrho_{V}\left(\varepsilon_{n}\right), & \varepsilon_{n} n \succ 0, \\ \vdots & \\ \varrho_{V}(-1), & -n \succ 0\end{cases}
$$

where $\varepsilon_{n}$ is a product of the fundamental units ${ }_{j} \epsilon, j=1, \ldots, d-1$, such that $\varepsilon_{n} n \succ 0$, this exists by our assumptions.

For the special case $d=2$ the fundamental unit $\epsilon>1$ and has $N \epsilon=-1$. Thus we can give $\varepsilon_{n}$ explicitly, if $n_{1}<0$ and $n_{2}>0$ the product $n_{1} \epsilon^{\prime}$ is totally positive where $\epsilon^{\prime}$ is the conjugate of $\epsilon$, and if $n_{1}>0$ and $n_{2}<0$ we choose $\varepsilon_{n}=\epsilon$.

Thus, there is at least one unit $\varepsilon$ such that $\varrho_{V}(\varepsilon) \neq 0$, otherwise we would have $\varphi_{V} \equiv 0$.

For $m, n \in \mathfrak{o}^{*}$, we have by 3.1.36

$$
t_{V}((m)) t_{V}((n))=\sum_{(d) \mid(m, n)} t_{V}\left(\left(\frac{m n}{d^{2}}\right)\right) .
$$

As in the modular case, Hecke operators $T_{\mathfrak{n}}$ are to be supplemented with involutions with which one can distinguish the parities for the cases in (3.1.37). To this end we define for any unit $\varepsilon$,

$$
\jmath_{\varepsilon}=\left(\left[\begin{array}{ll}
\operatorname{sgn}\left(\varepsilon_{1}\right) & \\
& 1
\end{array}\right], \ldots,\left[\begin{array}{ll}
\operatorname{sgn}\left(\varepsilon_{d}\right) & \\
& 1
\end{array}\right]\right) \in P G L_{2}(\mathbb{R})^{d}
$$

where $\varepsilon_{j}$ is the $j$-th embedding of $\varepsilon$. An easy computation shows that

$$
\jmath_{\varepsilon} G \jmath_{\varepsilon}=G, \quad\left(\mathrm{a}[|\varepsilon|] \jmath_{\varepsilon}\right) \Gamma\left(\mathrm{a}[|\varepsilon|] \jmath_{\varepsilon}\right)^{-1}=\Gamma .
$$

Then, for any $\Gamma$-automorphic function $f \in G$, we define

$$
\mathrm{i}_{\varepsilon} f(\mathrm{~g})=f\left(\mathrm{a}[|\varepsilon|] \jmath_{\varepsilon} \mathrm{g} \jmath_{\varepsilon}\right)=f\left(\mathrm{n}\left[\left(\varepsilon_{j} x_{j}\right)_{j}\right] \mathrm{a}\left[\left(\left|\varepsilon_{j}\right| y_{j}\right)_{j}\right] \mathrm{k}\left[\left(\operatorname{sgn}\left(\varepsilon_{j}\right) \theta_{j}\right)_{j}\right]\right) .
$$


By $(3.1 .39)$ we see that the left $\Gamma$-automorphy is preserved by $\mathrm{i}_{\varepsilon}$ and that

$$
\mathrm{i}_{\varepsilon}^{2}=1, \quad \mathrm{i}_{\varepsilon_{1}} \mathrm{i}_{\varepsilon_{2}}=\mathrm{i}_{\varepsilon_{1} \varepsilon_{2}}, \quad \Omega_{j} \mathrm{i}_{\varepsilon}=\mathrm{i}_{\varepsilon} \Omega_{j}, \quad T_{\mathfrak{n}} \mathrm{i}_{\varepsilon}=\mathrm{i}_{\varepsilon} T_{\mathfrak{n}},
$$

for $\varepsilon, \varepsilon_{1}, \varepsilon_{2} \in \mathfrak{o}^{\times}$.

But the weight of $f$ is in general not preserved: if $f$ is of weight $2 q$, the function $\mathrm{i}_{\varepsilon} f$ has weight $2\left(\operatorname{sgn}\left(\varepsilon_{1}\right) q_{1}, \ldots, \operatorname{sgn}\left(\varepsilon_{d}\right) q_{d}\right)$. To get an involution we must either restrict the weights, or choose $\varepsilon$ appropriately.

We can write

$$
\varrho_{V}\left(\varepsilon_{V} \varepsilon\right)=\varrho_{V}\left(\varepsilon_{V}\right) \lambda_{V}(\varepsilon)
$$

where $\lambda_{V}(\varepsilon) \in\{ \pm 1\} \cup\{0\}$ lives on the units modulo $P(\epsilon)^{2}=\prod_{j=1}^{d-1} \epsilon_{j}^{2}$. The unit $\varepsilon_{V}$ depends on $\operatorname{sgn}(q)$. For example, if $q=\underline{0}$ we have $\varepsilon_{V}=1$ and $\lambda_{V}$ is a real character of the unit group modulo $P(\epsilon)^{2}$.

In the case $d=2$ Bruggeman and Motohashi give explicit values for $\varepsilon_{V}$, namely $\varepsilon_{V}=1$ if $q_{V}=(0,0)$, and $\varepsilon_{V}=(-1)^{j-1} \operatorname{sgn}\left(q_{j}\right)$ if $q_{V}=\left(q_{j}, 0\right)$ or $\left(0, q_{j}\right)$ and $\varepsilon_{V}=\epsilon^{\frac{1+\operatorname{sgn}\left(q_{2}\right)}{2}} \epsilon^{\prime \frac{1-\operatorname{sgn}\left(q_{1}\right)}{2}}$ if $q_{V}=\left(q_{1}, q_{2}\right)$

Thus, we may put for $n \in \mathfrak{o}^{*}$ and any unit $\varepsilon$ such that $\varepsilon n \succ 0$

$$
\varrho_{V}(n)=\varrho_{V} \eta_{V}(n) t_{V}((n)), \quad \eta_{V}(n)=\lambda_{V}\left(\frac{\varepsilon}{\varepsilon_{V}}\right)
$$

where

$$
\varrho_{V}=\varrho_{V}\left(\varepsilon_{V}\right) .
$$

This definition of $\lambda_{*}$ via $i_{\varepsilon}$ is not limited to cuspidal subspaces. It can be extended to Eisenstein series as by the Fourier expansion given in (3.1.23) it holds that

$$
\mathrm{i}_{\varepsilon} E_{\underline{0}}(\mathrm{~g} ; s, \underline{\nu})=\left|\frac{\varepsilon_{1}}{\varepsilon_{d}}\right|^{i \nu_{1}} \ldots\left|\frac{\varepsilon_{d-1}}{\varepsilon_{d}}\right|^{i \nu_{d-1}} E_{\underline{0}}(\mathrm{~g} ; s, \underline{\nu}) .
$$

This, and the definition of $\mathcal{E}$ given in 3.1 .29 and 3.1 .30 lead to the extension of $\lambda_{*}$ and $\eta_{*}$ given by

$$
\lambda_{\varepsilon_{\underline{\nu}}}(\varepsilon)=\eta_{\varepsilon_{\underline{\nu}}}(\varepsilon)=\left|\frac{\varepsilon_{1}}{\varepsilon_{d}}\right|^{i \nu_{1}} \cdots\left|\frac{\varepsilon_{d-1}}{\varepsilon_{d}}\right|^{i \nu_{d-1}}= \pm 1 .
$$

\section{Geometric Sum Formula}

Now we are ready to state the spectral results that are essential for our purpose.

\section{(3.1.4) Lemma}

Let $\kappa_{V}=\left(\kappa_{1}, \ldots, \kappa_{d}\right)$ with $\kappa_{j}$ as in (3.1.31) and Lemma 3.1.3; $q_{V}=\left(q_{1}, \ldots, q_{d}\right)$ as defined in Lemma 3.1.3; $t_{V}((n))$ as defined by 3.1.35; $\eta_{V}(n)$ as defined by 3.1.40 and $\varrho_{V}$ as in 3.1.41. Let

$$
a_{V}:=\left.\left(\frac{2}{\pi}\right)^{d} \sqrt{\left|D_{K}\right|} \varrho_{V}\right|^{2} \prod_{j=1}^{d} \frac{\Gamma\left(\frac{1}{2}+\left|q_{j}\right|+i \overline{\kappa_{j}}\right)}{\Gamma\left(\frac{1}{2}+\left|q_{j}\right|+i \kappa_{j}\right)} .
$$

Further, let $w$ be a function defined for all $\kappa_{V}$ and satisfying $w\left(\kappa_{V}\right) \ll\left(\left(1+\left|\kappa_{1}\right|\right) \cdots\left(1+\left|\kappa_{d}\right|\right)\right)^{-2-\mu}$ with an arbitrary small constant $\mu>0$. 
Then we have for any $n \in \mathfrak{o}^{*}$,

$$
\sum_{V} a_{V}\left|\eta_{V}(n)\right| t_{V}((n))^{2} w\left(\kappa_{V}\right) \ll|N n|^{\frac{1}{2}+\mu}
$$

where $V$ runs over all cuspidal subspaces, and the implicit constant depends only on $\mu$.

This implies, in particular, that

$$
t_{V}((n)) \ll(N n)^{\frac{1}{4}+\mu}
$$

with the same dependency on $\mu$.

Proof.

This follows from [BMP01, equation 15, page 112].

\section{(3.1.5) Lemma}

Let $f$ be smooth over $(0, \infty)^{d}$, and $f$ and all its derivatives are of rapid decay if at least one variable tends to $0+$ or $+\infty$. Let

$$
\mathrm{B}_{\mathrm{e}} f\left(r_{1}, \ldots, r_{d}\right)=i^{d} \int \ldots \int_{(0, \infty)^{d}} \prod_{j=1}^{d} \frac{J_{2 i r_{j}}^{\left(e_{j}\right)}\left(u_{j}\right)-J_{-2 i r_{j}}^{\left(e_{j}\right)}\left(u_{j}\right)}{\sinh \left(\pi r_{j}\right)} f(\underline{u}) \frac{d \underline{u}}{N \underline{u}}
$$

where $\mathrm{e}=\left(e_{1}, \ldots, e_{d}\right)$ with $e_{j}= \pm 1,\left(r_{1}, \ldots, r_{d}\right) \in \mathbb{C}^{d}$, and $J_{\nu}^{+}=J_{\nu}, J_{\nu}^{-}=I_{\nu}$ are the Bessel functions defined in Section 5.2.

Then we have for any $m, n \in \mathfrak{o}^{*}$

$$
\begin{aligned}
& \sum_{c \in \mathfrak{o}^{*}} \frac{S_{K}(m, n ; c)}{N c} f\left(\left(\frac{4 \pi \sqrt{\left|m_{j} n_{j}\right|}}{\left|c_{j}\right|}\right)_{j}\right) \\
= & \sum_{V} a_{V} \eta_{V}(m) t_{V}((m)) \eta_{V}(n) t_{V}((n)) \mathrm{B}_{[m n]} f\left(\kappa_{V}\right) \\
& +C_{e} \sum_{\underline{\nu} \in \Lambda^{d-1}} \lambda_{-\underline{\nu}}(m n) \int_{-\infty}^{\infty} \frac{\sigma_{2 i t}(m, 2 \underline{\nu}) \sigma_{2 i t}(n, 2 \underline{\nu})}{|N(m n)|^{i t}\left|\zeta_{K}(1+2 i t, 2 \underline{\nu})\right|^{2}} \mathrm{~B}_{[m n]} f(t+\underline{\nu}) d t
\end{aligned}
$$

where $t+\underline{\nu}=\left(t+\nu_{1}, \ldots, t+\nu_{d-1}, t-\operatorname{Tr}(\underline{\nu})\right),[m n]=\left(\operatorname{sgn}\left(m_{1} n_{1}\right), \ldots, \operatorname{sgn}\left(m_{d} n_{d}\right)\right), C_{e}=2^{d} \sqrt{\left|D_{K}\right|}$ and the other symbols are as in the previous lemma. The functions $\zeta_{K}(s, \underline{\nu})$ and $\sigma_{\xi}(n, \underline{\nu})$ are defined in 3.1.5) and 3.1.9, and $S_{K}$ is defined in 2.0.2.

Proof.

For a proof see BMP01] Theorem 2.7.1 combined with Proposition 2.5.6 and 2.6.3 and B81, Proposition 14.2.8- 14.2.9].

We state an upper bound for $\mathrm{B}_{\mathrm{e}} f$ for later use. The right hand side of 3.1 .43 converges rapidly, as follows from the estimates of Lemma 3.1 .4 and the estimate

$$
\mathrm{B}_{\mathrm{e}} f(\underline{r}) \ll\left(1+\left|r_{1}\right|+\cdots+\left|r_{d}\right|\right)^{-\frac{C_{f}}{2}}
$$

for $\left|\operatorname{Im} r_{j}\right|<\frac{1}{4} C_{f}, j=1, \ldots, d$, and $C_{f}$ a large constant we specify in a moment.

For $f$ a smooth function with compact support, we can conclude this from [BMP01, Proposition 2.5.6]. 
But for a later use, we will deduce it by hand. Note that $\mathrm{B}_{\mathrm{e}} f(\underline{r})$ is regular for $\left|\operatorname{Im} r_{j}\right|<C_{f}$ for all $j$ and $C_{f}$ a large constant, see BMP01, Proposition 2.5.6, Definition 2.5.1]. We use the Mellin inversion theorem for suitable $a_{j}, j=1, \ldots, d$ and get

$$
f(\underline{u})=\frac{1}{(2 \pi i)^{d}} \int_{\left(a_{1}\right) \ldots\left(a_{d}\right)} \ldots \int \tilde{f}(\underline{s})\left(\frac{u_{1}}{2}\right)^{-2 s_{1}} \cdots\left(\frac{u_{d}}{2}\right)^{-2 s_{d}} d \underline{s}
$$

where

$$
\widetilde{f}(\underline{s})=\int_{(0, \infty)^{d}} \ldots \int_{f} f(\underline{u})\left(\frac{u_{1}}{2}\right)^{2 s_{1}-1} \cdots\left(\frac{u_{d}}{2}\right)^{2 s_{d}-1} d \underline{u}
$$

is the Mellin transform of $f$; it is holomorphic if $\left|\operatorname{Re} s_{j}\right|<C_{f}$ for all $j$, and is, in this region, of rapid decay.

Inserting the Mellin inversion in 3.1.42, we get

$$
\mathrm{B}_{\mathrm{e}} f(\underline{r})=\frac{i^{d} 2^{-d}}{(2 \pi i)^{d}} \int_{\left(a_{1}\right) \ldots\left(a_{d}\right)} \ldots \int_{\tilde{f}} \tilde{f}(\underline{s}) \prod_{j=1}^{d} J^{\left(e_{j}\right)}\left(s_{j}, r_{j}\right) d \underline{s}
$$

where $e_{j}= \pm$ and

$$
J^{(e)}(s, r)=\int_{0}^{\infty} \frac{J_{2 i r}^{(e)}(u)-J_{-2 i r}^{(e)}(u)}{\sinh (\pi r)}\left(\frac{u}{2}\right)^{-2 s-1} d u .
$$

If $\left|\operatorname{Im} r_{j}\right|<\frac{1}{4}$ for all $j=1, \ldots, d$ and $a_{j}$ satisfies $-\frac{1}{4}<a_{j}<-\left|\operatorname{Im} r_{j}\right|$, the right hand side of (3.1.46) converges absolutely, and the equality holds. Then for $-\frac{1}{4}<\operatorname{Re} s<-\left|\operatorname{Im} r_{j}\right|$ the function $J^{(e)}(s, r)$ equals

$$
J^{ \pm}(s, r)=\frac{1}{\pi i}\{(1 \pm 1) \cos (\pi s)+(1 \mp 1) \cosh (\pi r)\} \Gamma(i r-s) \Gamma(-i r-s) .
$$

In the case $e=+$ and $i r=l-\frac{1}{2}, l \in \mathbb{N}$, this changes to

$$
J^{+}\left(s, i\left(\frac{1}{2}-l\right)\right)=2 i(-1)^{l-1} \frac{\Gamma\left(l-\frac{1}{2}-s\right)}{\Gamma\left(l+\frac{1}{2}+s\right)} .
$$

Inserting this in 3.1.46) and shifting the contours to the left, we see that 3.1.46 holds for $\left|\operatorname{Im} r_{j}\right|<C_{f}$. Now shifting the contour to the right yields the estimate 3.1.44. Note that the poles we pass are of rapid decay in $r$ as $\tilde{f}$ is of rapid decay.

\section{Hecke Series}

We now introduce the Hecke series $H_{V}$ associated to the cuspidal irreducible subspaces $V$.

Let $t_{V}(\mathfrak{n})$ be as defined in 3.1.35, and put

$$
H_{V}(s)=\sum_{\mathfrak{n}} t_{V}(\mathfrak{n})(N \mathfrak{n})^{-s} .
$$


The Hecke series converges absolutely at least for $\operatorname{Re} s>\frac{5}{4}$, in this region it is bounded uniformly in $V$ as follows from Lemma 3.1.4. Using the Rankin $\zeta$-function attached to $V$ one can see that it converges for $\operatorname{Re} s>1$.

The multiplication formula 3.1.38 implies an Euler product for $H_{V}$ and in the region of absolute convergence we have the relation

$$
H_{V}\left(s_{1}\right) H_{V}\left(s_{2}\right)=\zeta_{K}\left(s_{1}+s_{2}\right) \sum_{\mathfrak{n}} \frac{\sigma_{s_{1}-s_{2}}(\mathfrak{n}) t_{V}(\mathfrak{n})}{(N \mathfrak{n})^{s_{1}}} .
$$

Further, we have a functional equation:

\section{(3.1.6) Lemma}

The Hecke series associated with a cuspidal irreducible subspace $V$ is an entire function satisfying the functional equation

$$
H_{V}(s)=H_{V}(1-s) \frac{(2 \pi)^{d(2 s-1)}}{\pi^{d}} \prod_{j=1}^{d}\left[\left(\lambda_{V}\left(\varepsilon_{j}\right) \cosh \left(\pi \kappa_{j}\right)-\cos (\pi s)\right) \Gamma\left(1-s+i \kappa_{j}\right) \Gamma\left(1-s-i \kappa_{j}\right)\right],
$$

where $\kappa_{V}=\left(\kappa_{1}, \ldots, \kappa_{d}\right)$ as above, and $\varepsilon_{j}$ is such that $n \varepsilon_{j} \succ 0$ if $n_{j}<0$ and $n_{i}>0$ for $i \neq j$.

In particular, we have the estimate

$$
H_{V}(s) \ll\left(1+|s|+\left|\kappa_{1}\right|+\cdots+\left|\kappa_{d}\right|\right)^{c}
$$

where $c=C(\operatorname{Re} s)$ depends only on $\operatorname{Re} s$, and the implicit constant depends on $K$ and $\operatorname{Re} s$.

Specifically, if $\operatorname{Re} s=\frac{1}{2}$ the convexity bound gives $c=\frac{1}{2}+\epsilon$.

Proof.

The functional equation is given in [JL70, Theorem 11.1], in the form $L(s, \pi)=\varepsilon(s, \pi) L(1-s, \tilde{\pi})$. To get the exact term that is named by $\varepsilon(s, \pi)$ in [JL70], we compute it directly.

We consider the case $q_{V}=\underline{0}$. The other cases are simpler, c.f. BM01, Proof of Lemma 4.4]. Let $\lambda_{j}=\frac{1}{2}\left(1-\lambda_{V}\left(\varepsilon_{j}\right)\right)$ for all $j=1, \ldots, d$, with $\varepsilon_{j}$ such that if $n_{j}<0$ and $n_{i}>0$ for all $i \neq j$, then $\left(n \varepsilon_{j}\right) \succ 0$. Let $\underline{\lambda}=\left(\lambda_{1}, \ldots, \lambda_{d}\right)$. Further define the function $\varphi_{V}^{*}=\left(\frac{1}{2}\left(\mathbf{e}_{1}^{+}-\mathbf{e}_{j}^{-}\right)\right)^{\lambda_{1}} \ldots\left(\frac{1}{2}\left(\mathbf{e}_{d}^{+}-\mathbf{e}_{j}^{-}\right)\right)^{\lambda_{d}} \varphi_{V}$ and

$$
A_{V}(s)=\int_{\substack{1 \leq \frac{y_{j}}{y_{d}} \leq \epsilon_{j} \epsilon^{4} \\ j=1, \ldots, d}} \ldots \varphi_{V}^{*}(\mathrm{a}[\underline{y}])(N \underline{y})^{s-\frac{3}{2}} d \underline{y}
$$

for Re $s$ sufficiently large.

Using the Fourier expansion and the relation 3.1 .40 with the special case $q=\underline{0}$, we have

$$
\varphi_{V}^{*}(\mathrm{a}[\underline{y}])=2^{d}(-2 \pi)^{\underline{\lambda}} \underline{y}^{\underline{\lambda}}(N \underline{y})^{\frac{1}{2}} \sum_{n \in \mathfrak{o}^{*}} \varrho_{V}^{*}(n) \prod_{j=1}^{d} \frac{K_{i \kappa_{j}}\left(2 \pi\left|n_{j}\right| y_{j}\right)}{\Gamma\left(\frac{1}{2}+i \kappa_{j}\right)}
$$

where $K_{\nu}$ is the Bessel $K$-function of order $\nu$, see Section 5.2, and

$$
\varrho_{V}^{*}(n)=\varrho_{V}(n) n^{\underline{\lambda}}=\varrho_{V}\left|n_{1}\right|^{\lambda_{1}} \cdots\left|n_{d}\right|^{\lambda_{d}} t_{V}((n)) .
$$


In fact, $\lambda_{V}(\varepsilon)$ is a non-trivial character of the unit group, provided $\lambda_{1}+\cdots+\lambda_{d} \neq 0$.

Inserting this in the definition of $A_{V}(s)$ we have

$$
\begin{aligned}
A_{V}(s)= & 2^{d} \varrho_{V}(-2 \pi)^{\lambda} \prod_{j=1}^{d} \frac{1}{\Gamma\left(\frac{1}{2}+i \kappa_{j}\right)} \times \\
& \times \sum_{n \bmod P(\epsilon)^{2}} t_{V}((n))\left|n_{1}\right|^{\lambda_{1}} \cdots\left|n_{d}\right|^{\lambda_{d}} \int \ldots \int_{(0, \infty)^{d}} \prod_{j=1}^{d} K_{i \kappa_{j}}\left(2 \pi\left|n_{j}\right| y_{j}\right) y_{j}^{\lambda_{j}+s-1} d \underline{y}
\end{aligned}
$$

where $P(\epsilon)={ }_{1} \epsilon{ }{ }_{d-1} \epsilon$. This integral converges absolutely at least for $\operatorname{Re} s>\frac{5}{4}$.

Evaluating the integral, we find that

$$
A_{V}(s)=\varrho_{V}(-1)^{\underline{\lambda}} 2^{\underline{\lambda}-d} \pi^{-d s} \prod_{j=1}^{d} \frac{\Gamma\left(\frac { 1 } { 2 } ( \lambda _ { j } + s + i \kappa _ { j } ) \Gamma \left(\frac{1}{2}\left(\lambda_{j}+s-i \kappa_{j}\right)\right.\right.}{\Gamma\left(\frac{1}{2}+i \kappa_{j}\right)} H_{V}(s) .
$$

On the other hand, with the same argument as in the proof of Lemma 3.2.1 we have

$$
\varphi_{V}^{*}(\mathrm{a}[\underline{y}])=(-1)^{\underline{\lambda}} \varphi_{V}^{*}\left(\mathrm{a}[\underline{y}]^{-1}\right)
$$

Then dividing the integral into two pieces, namely $N \underline{y} \leq 1$ and $N \underline{y} \geq 1$, we see that $A_{V}(s)$ is an entire function, and satisfies the functional equation $A_{V}(s)=(-1)^{\lambda} A_{V}(1-s)$.

Using 3.1 .50 we get the functional equation as claimed.

The second statement follows from the functional equation and the estimate on $t_{V}((n))$ given in Lemma 3.1.4 by using the Phragmén-Lindelöf convexity principle. 


\subsection{Spectral Decomposition of The Fourth Moment of $\zeta_{K}$}

The goal of this section is to find a spectral decomposition for

$$
\mathcal{Z}_{2}(g, \omega ; K):=\sum_{\underline{\nu} \in \Lambda^{d-1}} \omega(\underline{\nu}) \int_{-\infty}^{\infty}\left|\zeta_{K}\left(\frac{1}{2}+i t, \underline{\nu}\right)\right|^{4} g(t) d t
$$

where $g$ and $\omega$ are assumed to be entire, real valued on $\mathbb{R}$, and of rapid decay in any fixed horizontal strip in every variable $t, \nu_{j}$. The number field $K$ is as in Chapter 2.

Bruggeman and Motohashi have done this in their paper BM01 for the special case $d=2$, this chapter follows closely their arguments.

\subsubsection{Initial Reduction}

To begin we set for $\operatorname{Re} z_{j}>1, j=1, \ldots, 4$ :

$$
\begin{aligned}
& \mathcal{J}\left(z_{1}, z_{2}, z_{3}, z_{4} ; g, \omega\right) \\
& =\sum_{\underline{\nu} \in \Lambda^{d-1}} \omega(\underline{\nu}) \int_{-\infty}^{\infty} \zeta_{K}\left(z_{1}+i t, \underline{\nu}\right) \zeta_{K}\left(z_{2}+i t, \underline{\nu}\right) \zeta_{K}\left(z_{3}-i t,-\underline{\nu}\right) \zeta_{K}\left(z_{4}-i t,-\underline{\nu}\right) g(t) d t .
\end{aligned}
$$

The function $\mathfrak{J}\left(z_{1}, z_{2}, z_{3}, z_{4}\right)$ has a meromorphic continuation to $\mathbb{C}^{4}$. In particular, using the estimate (3.1.6) we see that it is regular at $p_{\frac{1}{2}}=\left(\frac{1}{2}, \frac{1}{2}, \frac{1}{2}, \frac{1}{2}\right)$.

To obtain the continuation, let $\operatorname{Re} z_{j}>1$ for all $j$. Moving the path of integration upwards, we collect two residues coming from the poles at $t=\left(z_{1}-1\right) i$ and $t=\left(z_{2}-1\right) i$ if $\underline{\nu}=\underline{0}$ and we see that $\mathcal{J}$ is a meromorphic function in a region $\left|z_{j}\right|<B$ for some $B$ depending on $g$ and $\omega$. Now let $\operatorname{Re} z_{j}<1$ for all $j$ and move the path back to the original, collecting again two residues at $t=\left(1-z_{3}\right) i$ and $t=\left(1-z_{4}\right) i$ if $\underline{\nu}=\underline{0}$. Then we get for $\operatorname{Re} z_{j}<1, j=1, \ldots, 4$,

$$
\begin{aligned}
\mathcal{J}\left(z_{1}, z_{2}, z_{3}, z_{4} ; g, \omega\right)= & \sum_{\underline{\nu} \in \Lambda^{d-1}} \omega(\underline{\nu}) \int_{-\infty}^{\infty} \zeta_{K}\left(z_{1}+i t, \underline{\nu}\right) \zeta_{K}\left(z_{2}+i t, \underline{\nu}\right) \zeta_{K}\left(z_{3}-i t,-\underline{\nu}\right) \zeta_{K}\left(z_{4}-i t,-\underline{\nu}\right) g(t) d t \\
& +2 \pi \omega(\underline{0}) \zeta_{K}\left(z_{2}-z_{1}+1, \underline{0}\right) \zeta_{K}\left(z_{1}+z_{3}-1, \underline{0}\right) \zeta_{K}\left(z_{1}+z_{4}-1, \underline{0}\right) g\left(\left(z_{1}-1\right) i\right) \\
& +2 \pi \omega(\underline{0}) \zeta_{K}\left(z_{1}-z_{2}+1, \underline{0}\right) \zeta_{K}\left(z_{2}+z_{3}-1, \underline{0}\right) \zeta_{K}\left(z_{2}+z_{4}-1, \underline{0}\right) g\left(\left(z_{2}-1\right) i\right) \\
& +2 \pi \omega(\underline{0}) \zeta_{K}\left(z_{4}-z_{3}+1, \underline{0}\right) \zeta_{K}\left(z_{1}+z_{3}-1, \underline{0}\right) \zeta_{K}\left(z_{2}+z_{3}-1, \underline{0}\right) g\left(\left(1-z_{3}\right) i\right) \\
& +2 \pi \omega(\underline{0}) \zeta_{K}\left(z_{3}-z_{4}+1, \underline{0}\right) \zeta_{K}\left(z_{1}+z_{4}-1, \underline{0}\right) \zeta_{K}\left(z_{2}+z_{4}-1, \underline{0}\right) g\left(\left(1-z_{4}\right) i\right) .
\end{aligned}
$$

Now we insert the point $p_{\frac{1}{2}}$ and get

$$
\mathcal{Z}_{2}(g, \omega ; K)=\mathcal{J}\left(p_{\frac{1}{2}} ; g, \omega\right)+C_{K}(g, \omega)
$$

where the constant $C_{K}(g, \omega)$ comes from the terms $g\left( \pm\left(z_{j}-1\right) i\right)$ by considering the Laurent series of $\zeta_{K}\left( \pm\left(\left(z_{j^{\prime}}-z_{j}\right)+1\right) i\right)$. We have

$$
C_{K}(g, \omega)=\omega(\underline{0})\left(a_{0} g\left(\frac{1}{2} i\right)+b_{0} g\left(-\frac{1}{2} i\right)+a_{1} g^{\prime}\left(\frac{1}{2} i\right)+b_{1} g^{\prime}\left(-\frac{1}{2} i\right)\right)
$$

with constants $a_{0}, b_{0}, a_{1}, b_{1}$ depending on $K$ that can be made explicit. 
Now we consider $\mathcal{J}\left(z_{1}, z_{2}, z_{3}, z_{4} ; g, \omega\right)$ in the domain $\operatorname{Re} z_{j}>1$ for all $j$, so that the integral converges absolutely.

In this region of absolute convergence, we use the definitions in (3.2.1) and insert the partition of unity $\Psi_{+}$defined in (3.1.13). Further we use the identity (3.1.10) to obtain

$$
\begin{aligned}
\mathcal{J}\left(z_{1}, z_{2}, z_{3}, z_{4} ; g, \omega\right) & =\sum_{\underline{\nu} \in \Lambda^{d-1}} \omega(\underline{\nu}) \int_{-\infty}^{\infty} \sum_{a, b \in \mathfrak{o}^{*}} \frac{\sigma_{z_{1}-z_{2}}(a) \sigma_{z_{3}-z_{4}}(b)}{(N a)^{z_{1}+i t}(N b)^{z_{3}-i t}} \Psi_{+}(a) \Psi_{+}(b) \lambda_{\underline{\nu}}\left(\frac{a}{b}\right) g(t) d t \\
& =\sum_{a, b \in \mathfrak{o}^{*}} \frac{\sigma_{z_{1}-z_{2}}(a) \sigma_{z_{3}-z_{4}}(b)}{(N a)^{z_{1}}(N b)^{z_{3}}} \Psi_{+}(a) \Psi_{+}(b) W\left(\frac{b}{a}\right)
\end{aligned}
$$

where the function $W$ is given by

$$
W(x)=\widehat{g}(\log (N x)) \frac{1}{\operatorname{vol}\left(\Lambda^{d-1}\right)} \sum_{\underline{h} \in \widehat{\Lambda}^{d-1}} \widehat{\omega}\left(\left(h_{j}+\log \left(\frac{x_{d}}{x_{j}}\right)\right)_{j=1}^{d-1}\right)
$$

for $x \succ 0$. If $x \nsucc 0$ the function $W(x)$ vanishes. The lattice $\widehat{\Lambda}^{d-1}$ is the dual lattice of $\Lambda^{d-1}$.

The sum $\sum_{a, b \in \mathfrak{o}^{*}}$ splits into two parts, namely $\sum_{a=b}$ and $\sum_{a \neq b}$. We denote them by $\mathcal{J}_{0}$ and $\mathcal{J}_{+}$ respectively, and have $\mathcal{J}\left(z_{1}, z_{2}, z_{3}, z_{4} ; g, \omega\right)=\left\{\mathcal{J}_{0}+\mathcal{J}_{+}\right\}\left(z_{1}, z_{2}, z_{3}, z_{4} ; g, \omega\right)$.

Due to 3.1 .15 the sum $\mathfrak{J}_{0}$ equals

$$
\mathcal{J}_{0}\left(z_{1}, z_{2}, z_{3}, z_{4} ; g, \omega\right)=2^{1-d} c_{\Psi} W(\underline{1}) \frac{\zeta_{K}\left(z_{1}+z_{3}\right) \zeta_{K}\left(z_{1}+z_{4}\right) \zeta_{K}\left(z_{2}+z_{3}\right) \zeta_{K}\left(z_{2}+z_{4}\right)}{\zeta_{K}\left(z_{1}+z_{2}+z_{3}+z_{4}\right)} .
$$

Now we consider the term $\mathcal{J}_{+}$. Let $a=n$ and $b=n+m$. As $a, b \succ 0$ we have $n, n+m \succ 0$, and $\mathcal{J}_{+}$ becomes

$$
\mathcal{J}_{+}\left(z_{1}, z_{2}, z_{3}, z_{4} ; g, \omega\right)=\sum_{m \in \mathfrak{o}^{*}}(N m)^{-z_{1}-z_{3}} \sum_{\substack{n \in \mathfrak{o}^{*} \\ n+m \neq 0}} \frac{\sigma_{z_{1}-z_{2}}(n) \sigma_{z_{3}-z_{4}}(n+m)}{\left|N\left(\frac{n}{m}\right)\right|^{z_{1}}\left|N\left(1+\frac{n}{m}\right)\right|^{z_{3}}} W\left(1+\frac{m}{n}\right) \Psi_{+}(n) \Psi_{+}(n+m) .
$$

We rewrite $m \in \mathfrak{o}^{*}$ as $\varepsilon m$, with $\varepsilon \in \mathfrak{o}^{\times}$and $m \in \mathcal{F}$, and also split off a factor $\varepsilon$ from $n$. Thus the sum over the units can be separated from the innermost sum, and formula 3.1 .12 gives

$$
\sum_{\varepsilon \in \mathfrak{o}^{\times}} \Psi_{+}(\varepsilon n) \Psi_{+}(\varepsilon(n+m))=c_{\Psi}\left(1+\frac{m}{n}\right)
$$

where

$$
c_{\Psi}(x)=\frac{1}{(8 \pi)^{d-1}} \int_{\mathbb{R}^{d-1}} \ldots \int \widehat{\mathrm{p}}(\underline{\xi})^{2} \exp \left(i \sum_{j=1}^{d-1} \xi_{j} \log \left|\frac{x_{j}}{x_{d}}\right| \bar{\nu}_{j}\right) d \xi
$$

thus $\frac{1}{2^{d-1}} c_{\Psi}=c_{\Psi}(1)$.

For sufficiently nice $h$ that are specified later, let

$$
\mathcal{B}_{m}(\alpha, \beta ; h):=\sum_{\substack{n \in 0^{*} \\ n+m \neq 0}} \sigma_{\alpha}(n) \sigma_{\beta}(n+m) h\left(\frac{n}{m}\right), \quad m \succ 0
$$


Then in the region of absolute convergence, i.e. $\operatorname{Re} z_{j}>1$ for all $j$, the function $\mathcal{J}_{+}$can be written as

$$
\mathcal{J}_{+}\left(z_{1}, z_{2}, z_{3}, z_{4} ; g, \omega\right)=\sum_{m \in \mathcal{F}}(N m)^{-z_{1}-z_{3}} \mathcal{B}_{m}\left(z_{1}-z_{2}, z_{3}-z_{4} ; W_{+}\left(\cdot ; z_{1}, z_{3}\right)\right)
$$

where the function $W_{+}$is given by

$$
W_{+}(y ; \gamma, \delta)=\frac{W\left(1+\frac{1}{y}\right) c_{\Psi}\left(1+\frac{1}{y}\right)}{|N(y)|^{\gamma}|N(1+y)|^{\delta}}
$$

Its support is contained in the region $1+\frac{1}{y} \succ 0$, as $W(x)$ is supported on $x \succ 0$.

Our aim is to transform $\mathcal{B}_{m}(\alpha, \beta ; h)$, in the region of absolute convergence, into a sum over Kloosterman sums over $K$. Afterwards we decompose it spectrally with the geometric sum formula given in Section 3.1.2 provided that $h$ satisfies the condition (3.2.17). Then we continue it to a neighborhood of $p_{\frac{1}{2}}$. Finally we check that $W$ satisfies this condition and use the decomposition of $\mathcal{B}_{m}(\alpha, \beta ; h)$ to spectrally decompose $\mathcal{Z}_{2}(g, \omega ; K)$.

To begin we use the Ramanujan expansion 3.1 .26 with the character $\underline{\nu}=\underline{0}$ to separate the parameters $n$ and $m$ in $\sigma_{\beta}(n+m)$ in $\mathcal{B}_{m}(\alpha, \beta ; h)$. Recall the definition of $e[x]$ for $x \in K$, given in (2.0.1). Thus, for $\operatorname{Re} \beta<-1$,

$$
\mathcal{B}_{m}(\alpha, \beta ; h)=\zeta_{K}(1-\beta) \sum_{c \in \mathfrak{o}^{*}} \Psi(c)|N c|^{\beta-1} \sum_{\substack{a \bmod c \\(a, c)=1}} e\left[\frac{a m}{c}\right] \mathcal{D}_{m}\left(\alpha, \frac{a}{c} ; h\right)
$$

where

$$
\mathcal{D}_{m}\left(\alpha, \frac{a}{c} ; h\right):=\sum_{n \in \mathfrak{o}^{*} / \mathrm{U}_{c}} \sigma_{\alpha}(n) e\left[\frac{a n}{c}\right] h\left(\frac{n}{m} ; c\right)
$$

with

$$
h(x ; c)=\sum_{\varepsilon \in \mathrm{U}_{c}} h(\varepsilon x)
$$

and $\mathrm{U}_{c}=\{\varepsilon \mid$ totally positive unit congruent to $1 \bmod c\}$. Let ${ }_{1} \epsilon_{c}, \ldots,{ }_{d-1} \epsilon_{c}$ be a system of generators of $\mathrm{U}_{c}$ with ${ }_{j} \epsilon_{c}>1$, and ${ }_{j} \epsilon_{c} \succ 0$. Then every element $\varepsilon \in \mathrm{U}_{c}$ is of the form

$$
\varepsilon=\prod_{j=1}^{d-1}{ }_{j} \epsilon_{c}^{\nu_{j}}
$$

where $\nu_{j} \in \mathbb{Z}$.

We assume $h$ to be continuous and such that 3.2.11) converges absolutely. For $x \in K$ let the function $h(x ; c)$ be regular and for $N x$ large, be bounded by

$$
h(x ; c) \ll|N x|^{-1-\max \{0, \operatorname{Re} \alpha\}-\mu}
$$

uniformly in $c$, for some $\mu>0$. Further let $h(x ; c)$ be of rapid decay if at least one variable tends to 0 or -1 . Under these conditions the expansion $(3.2 .9)$ of $\mathcal{B}_{m}$ holds for $\operatorname{Re} \beta<-1$. 
After separation of the parameters $n$ and $m$ in $\sigma_{\beta}(n+m)$, we need the analogous separation for $h\left(\frac{n}{m} ; c\right)$, too. To this end we extend $h$ to $(\mathbb{R} \backslash\{0\})^{d}$ via $x \mapsto\left(x_{1}, \ldots, x_{d}\right)$, following BM01, such that

$$
h(\underline{x} ; c)=h\left(x_{1}, \ldots, x_{d} ; c\right)=\sum_{\underline{\nu} \in \mathbb{Z}^{d-1}} h\left(\left(\epsilon_{c}^{\nu_{1}} \cdots{ }_{d-1} \epsilon_{c}^{\nu_{d-1}} x\right)_{j=1}^{d}\right) .
$$

Note that $\left({ }_{1} \epsilon_{c}^{\nu_{1}} \cdots_{d-1} \epsilon_{c}^{\nu_{d-1}}\right)_{d}=\prod_{j=1}^{d-1}\left({ }_{1} \epsilon_{c}^{\nu_{1}} \cdots_{d-1} \epsilon_{c}^{\nu_{d-1}}\right)_{j}^{-1}$

As the sum in 3.2.11 converges absolutely, the sum over $\underline{\nu}$ converges absolutely, too. Thus the function $h\left(\left({ }_{1} \epsilon_{c}^{\nu_{1}} \cdots{ }_{d-1} \epsilon_{c}^{\nu_{d-1}} x\right)_{j=1}^{d}\right)$ is of rapid decay at infinity and we can apply Poisson's summation formula. If we substitute $\left({ }_{1} \epsilon_{c}^{t_{1}} \cdots{ }_{d-1} \epsilon_{c}^{t_{d-1}}\right)_{j} \mapsto \xi_{j}$ for $j=1, \ldots, d-1$, then the variables $t_{j}$ and $\xi_{i}$ satisfy some linear equation and one can express $t_{j}=R_{K}^{-1} \sum_{i=1}^{d-1} c_{j, i} \log \xi_{i}$, where the constant $c_{j, i}$ is a sum of the minors $\left(\log \left(k_{k} \epsilon_{c}\right)_{l}\right)_{j i}$, with $l \neq i$ and $k \neq j$. Then the exponential function $e^{i t \underline{\nu}}$ changes to $\xi_{j}^{R_{K}^{-1} \sum_{i=1}^{d-1} c_{i, j} \nu_{i}}$, and we view the new exponents $\underline{\tilde{\nu}}$ as elements of a $(d-1)$-dimensional lattice $\Lambda_{c}^{d-1}$. We have

$$
h(\underline{x} ; c)=\operatorname{vol}\left(\Lambda_{c}^{d-1}\right) \sum_{\underline{\tilde{\nu}} \in \Lambda_{c}^{d-1}} \int_{(0, \infty)^{d-1}} \ldots \int_{1} h\left(\xi_{1} x_{1}, \ldots, \xi_{d-1} x_{d-1}, \xi_{1}^{-1} \cdots \xi_{d-1}^{-1} x_{d}\right) \xi_{j}^{-i \tilde{\nu}_{j}} \frac{d \underline{\xi}}{N \xi} .
$$

Let $x_{j}=e_{j}\left|x_{j}\right|$ with $e_{j}= \pm 1$. Then changing variables $\xi_{j} \mapsto \xi_{j}|N \underline{x}|^{\frac{1}{d}} /\left|x_{j}\right|$ gives

$$
\left.h(\mathrm{e}|\underline{x}| ; c)=\operatorname{vol}\left(\Lambda_{c}^{d-1}\right) \sum_{\underline{\tilde{\nu}} \in \Lambda_{c}^{d-1}} \lambda_{\underline{\tilde{\nu}}}(\underline{x}) \int_{(0, \infty)^{d-1}} \ldots \int_{\int^{\prime}} h\left(e_{j} \xi_{j}|N(\underline{x})|^{\frac{1}{d}}\right)_{j=1}^{d-1}, e_{d} \prod_{j=1}^{d-1} \xi_{j}^{-1}|N(\underline{x})|^{\frac{1}{d}}\right) \xi_{j}^{-i \tilde{\nu}_{j}} \frac{d \underline{\xi}}{N \xi} .
$$

Now the integral is a function of $|N \underline{x}|^{\frac{1}{d}}$.

Using the Mellin inversion theorem in each orthant separately, we have

$$
h(\mathrm{e}|\underline{x}| ; c)=\frac{\operatorname{vol}\left(\Lambda_{c}^{d-1}\right)}{2 \pi i} \sum_{\underline{\tilde{\nu}} \in \Lambda_{c}^{d-1}} \lambda_{\tilde{\underline{\nu}}}(\underline{x}) \int_{(v)} \widetilde{h}\left(s-i \tilde{\nu}_{1}, \ldots, s-i \tilde{\nu}_{d-1}, s+i \operatorname{Tr}(\underline{\tilde{\nu}}) ; \mathrm{e}\right)|N(\underline{x})|^{-s} d s
$$

where $\mathrm{e}=\left(e_{1}, \ldots, e_{d}\right)$ and $\widetilde{h}$ is the Mellin transform of $h$, given by

$$
\widetilde{h}(\underline{s} ; \mathrm{e})=\int_{(0, \infty)^{d}} \ldots \int_{j} h(\mathrm{e} \underline{u}) \prod_{j=1}^{d} u_{j}^{s_{j}-1} d \underline{u} .
$$

The requirements to do so are satisfied for $1+\max \{0, \operatorname{Re} \alpha\}<v$ by the bound of $h$ given in 3.2.12.

For $1+\max \{0, \operatorname{Re} \alpha\}<v$ a rearrangement yields

$$
\begin{aligned}
& \mathcal{D}_{m}\left(\alpha, \frac{a}{c} ; h\right) \\
= & \frac{\operatorname{vol}\left(\Lambda_{c}^{d-1}\right)}{2 \pi i} \sum_{\ell} \sum_{\underline{\tilde{v}} \in \Lambda_{c}^{d-1}} \lambda_{-\underline{\tilde{\nu}}}(m) \int_{(v)}|N(m)|^{s} \widetilde{h}_{\ell}\left(s-i \tilde{\nu}_{1}, \ldots, s-i \tilde{\nu}_{d-1}, s+i \operatorname{Tr}(\underline{\tilde{\nu}})\right) D_{\ell}\left(s, \alpha ; \underline{\tilde{\nu}} ; \frac{a}{c}\right) d s
\end{aligned}
$$

where the sum over $\ell$ runs over the vectors $\left(l_{1}, \ldots, l_{d}\right)$ with $l_{j}=0,1$. 
The functions $\widetilde{h}_{\ell}$ and $D_{\ell}$ are defined by

$$
\begin{aligned}
\widetilde{h}_{\ell}(\underline{s}) & =\frac{1}{2^{d}} \sum_{\mathrm{e}=\left(e_{1}, \ldots, e_{d}\right)} \mathrm{e}^{\ell} \widetilde{h}(\underline{s} ; \mathrm{e}), \\
D_{\ell}\left(s, \alpha ; \underline{\tilde{\tilde{\nu}}} ; \frac{a}{c}\right) & =\sum_{n \in \mathfrak{o}^{*} / \mathrm{U}_{c}} \frac{\sigma_{\alpha}(n)}{|N n|^{s}} \lambda_{\underline{\tilde{\nu}}}(n) \operatorname{sgn}^{\ell}[n] e\left[\frac{a n}{c}\right] .
\end{aligned}
$$

with $\operatorname{sgn}^{\ell}[x]=\operatorname{sgn}^{\ell}\left[x_{1}, \ldots, x_{d}\right]=\prod_{j=1}^{d}\left(\frac{x_{j}}{\left|x_{j}\right|}\right)^{l_{j}}$.

We assume that $h$ is continuous and of rapid decay if at least one variable tends to infinity, zero or -1 and that

$$
\begin{aligned}
& \widetilde{h} \text { is regular and } \\
& \widetilde{h}(\underline{s} ; \mathrm{e}) \ll\left(1+\left|s_{1}\right|+\cdots+\left|s_{d}\right|\right)^{-C_{0}} \quad \text { in }\left|\operatorname{Re} s_{j}\right|<C_{0} \text {, for all } j=1, \ldots, d
\end{aligned}
$$

for a sufficiently large $C_{0}>0$. Then condition 3.2 .12 holds for $|\operatorname{Re} \alpha|<\frac{1}{2} C_{0}$. In particular 3.2.14 holds for an appropriate contour $(v)$.

\subsubsection{Kloosterman Sums}

In this section we deduce a functional equation for the function $D_{\ell}\left(s, \alpha ; \underline{\nu} ; \frac{a}{c}\right)$ defined in 3.2 .16 and use it to represent $\mathcal{B}_{m}$ as a sum over Kloosterman sums over $K$.

\section{(3.2.1) Lemma}

The function $D_{\ell}$ has a meromorphic continuation to $\mathbb{C}$. If $\alpha \neq 0$ it has at worst simple poles at $s=1,1+\alpha$ with residues

$$
\frac{2^{d} \delta_{\underline{\underline{\nu}}, \underline{\underline{0}}} \delta_{\ell, \underline{0}}}{\operatorname{vol}\left(\Lambda_{c}^{d-1}\right)}\left|D_{K}\right|^{-\frac{\alpha}{2}+\frac{1}{4}}|N c|^{\alpha-1} \zeta_{K}(1-\alpha), \quad \frac{2^{d} \delta_{\underline{\underline{\nu}}, \underline{0}} \delta_{\ell, \underline{0}}}{\operatorname{vol}\left(\Lambda_{c}^{d-1}\right)}\left|D_{K}\right|^{\frac{\alpha}{2}-\frac{1}{4}}|N c|^{-\alpha-1} \zeta_{K}(1+\alpha) .
$$

In particular $D_{\ell}$ is entire unless $\underline{\nu}=\underline{0}$. Furthermore we have

$$
D_{\ell}\left(s, \alpha ; \underline{\nu} ; \frac{a}{c}\right)=\frac{1}{\pi^{d}}\left(\frac{(2 \pi)^{d}}{|N c|}\right)^{2 s-\alpha-1} \lambda_{2 \underline{\nu}}(c) \Gamma_{\ell}(s-i \underline{\nu} ; \alpha) D_{\ell}\left(1-s,-\alpha ;-\underline{\nu} ; \frac{a^{*}}{c}\right)
$$

where $a a^{*} \equiv 1(\bmod c), s-i \underline{\nu}=\left(s-i \nu_{1}, \ldots, s-i \nu_{d-1}, s+i \operatorname{Tr}(\underline{\nu})\right)$, and

$$
\Gamma_{\ell}(\underline{s} ; \alpha)=\prod_{j=1}^{d}\left[\cos \left(\frac{\pi}{2} \alpha\right)-(-1)^{l_{j}} \cos \left(\pi\left(s_{j}-\frac{1}{2} \alpha\right)\right)\right] \Gamma\left(1-s_{j}\right) \Gamma\left(1+\alpha-s_{j}\right) .
$$

Proof.

Fix $\ell$ and define

$$
f_{\alpha}\left(\mathrm{g} ; \frac{a}{c}\right)=\pi^{-d \frac{\alpha}{2}}\left|D_{K}\right|^{\frac{\alpha}{2}-\frac{1}{4}} \Gamma\left(\frac{1}{2}(1+\alpha)\right)^{d} \zeta_{K}(1+\alpha) \prod_{j=1}^{d}\left(\mathbf{e}_{j}^{+}-\mathbf{e}_{j}^{-}\right)^{l_{j}} E_{\underline{0}}\left(\mathrm{n}\left[\frac{a}{c}\right] \mathrm{g} ; \frac{1}{2} \alpha, \underline{0}\right) .
$$

Recall that $E_{\underline{0}}(\mathrm{~g} ; s, \underline{\nu})$ is defined in 3.1 .22

Choose $\mathrm{g}=\mathrm{a}[\underline{y}]^{-1}$. An easy computation shows

$$
\mathrm{n}\left[\frac{a}{c}\right] \mathrm{a}[\underline{y}]^{-1} \in \operatorname{\Gamma n}\left[\frac{-a^{*}}{c}\right] \mathrm{a}\left[\underline{y} / c^{2}\right] \mathrm{w}
$$


where $\mathrm{w}$ is defined in 3.1.16. Thus, as the Eisenstein series $E_{\underline{0}}$ is a $\Gamma$-automorphic form as are $\mathbf{e}_{j}^{ \pm} E$, we have

$$
f_{\alpha}\left(\mathrm{a}[\underline{y}]^{-1} ; \frac{a}{c}\right)=(-1)^{\operatorname{Tr}(\ell)} f_{\alpha}\left(\mathrm{a}\left[\underline{y} / c^{2}\right] ; \frac{a}{c}\right) .
$$

On the other hand, we use the Fourier expansion of the Eisenstein series, stated in Lemma 3.1.2. This gives the expression

$$
\begin{aligned}
f_{\alpha}\left(\mathrm{a}[\underline{y}] ; \frac{a}{c}\right)= & \delta_{\ell, 0}\left(\pi^{-d \frac{\alpha}{2}}\left|D_{K}\right|^{\frac{\alpha}{2}-\frac{1}{4}} \Gamma\left(\frac{1}{2}(1+\alpha)\right)^{d} \zeta_{K}(1+\alpha)(N \underline{y})^{\frac{1}{2}(1+\alpha)}\right. \\
& \left.+\pi^{d \frac{\alpha}{2}}\left|D_{K}\right|^{-\frac{\alpha}{2}+\frac{1}{4}} \Gamma\left(\frac{1}{2}(1-\alpha)\right)^{d} \zeta_{K}(1-\alpha)(N \underline{y})^{\frac{1}{2}(1-\alpha)}\right) \\
& +(-1)^{\ell}(4 \pi)^{\ell+\frac{d}{2}} \underline{y}^{\ell}(N \underline{y})^{\frac{1}{2}} \sum_{n \in \mathfrak{o}^{*}} \frac{\sigma_{\alpha}(n)}{(N n)^{\frac{1}{2} \alpha}} n^{\ell} e\left[\frac{a n}{c}\right] \prod_{j=1}^{d} K_{\frac{\alpha}{2}}\left(2 \pi\left|n_{j}\right| y_{j}\right) \\
= & :\left\{f_{\alpha}^{(0)}+f_{\alpha}^{(1)}\right\}\left(\mathrm{a}[\underline{y}] ; \frac{a}{c}\right)
\end{aligned}
$$

where $K_{\mu}$ is the $K$-Bessel function of order $\mu$.

Then we consider the integral over $f_{\alpha}^{(1)}$ given by

$$
I\left(s, \alpha ; \underline{\nu} ; \frac{a}{c}\right)=\int_{1 \leq \frac{y_{1} \cdots y_{d-1}}{y_{d}} \leq P\left(\epsilon_{c}\right)^{2}} f_{\alpha}^{(1)}\left(\mathrm{a}\left[\underline{y}|c|^{-1}\right] ; \frac{a}{c}\right)(N \underline{y})^{s-\frac{1}{2}(\alpha+3)} \lambda_{\underline{\nu}}(\underline{y}) d \underline{y}
$$

where $P\left(\epsilon_{c}\right):=\prod_{j, k=1}^{d-1}\left({ }_{j} \epsilon_{c}\right)_{k}$. We shall see that up to some factors this integral is equal to the function $D_{\ell}$. Thus, computing a functional equation for it gives the functional equation for $D_{\ell}$. The details are presented in the following steps.

We insert the definition of $f_{\alpha}^{(1)}$ and change the summation over $n \in \mathfrak{o}^{*}$ into the two sums $n \in \mathfrak{o}^{*} / U_{c}$ and $\underline{p} \in \mathbb{Z}^{d-1}$. Then we have

$$
\begin{aligned}
I\left(s, \alpha ; \underline{\nu} ; \frac{a}{c}\right)= & (-1)^{\ell}(4 \pi)^{\ell+\frac{d}{2}}\left|D_{K}\right|^{\frac{\alpha}{2}-\frac{1}{4}}|c|^{-\ell}|N c|^{-\frac{1}{2}} \sum_{n \in \mathfrak{o}^{*} / U_{c}} \frac{\sigma_{\alpha}(n)}{|N n|^{\frac{\alpha}{2}}} n^{\ell} e\left[\frac{a n}{c}\right] \int_{1 \leq \frac{y_{1} \cdots y_{d-1}}{y_{d}} \leq P\left(\epsilon_{c}\right)^{2}} \cdots \int_{\underline{p} \in \mathbb{Z}^{d-1}} \times \\
& \left.\times \prod_{j=1}^{d} K_{\frac{\alpha}{2}}\left(2 \pi\left({ }_{1} \epsilon_{c}^{p_{1}} \cdots{ }_{d-1} \epsilon_{c}^{p_{d-1}}\right)_{j} y_{j}\left|\frac{n_{j}}{c_{j}}\right|\right)\left({ }_{1} \epsilon_{c}^{p_{1}} \cdots{ }_{d-1} \epsilon_{c}^{p_{d-1}}\right)_{j} y_{j}\right)^{s+l_{j}-1-\frac{\alpha}{2}-i \nu_{j}} d \underline{y} .
\end{aligned}
$$

As usual $\nu_{d}=-\operatorname{Tr}(\underline{\nu})$.

By the exponential decay of the Bessel $K$-function for large arguments and its behaviour for small arguments, these integrals converge absolutely. Hence, we can unfold the integral with the sum. Using [GR07, 6.561.16] to compute the resulting integral, we see that $I$ is essentially $D_{\ell}$, more precisely

$$
\begin{aligned}
I\left(s, \alpha ; \underline{\nu} ; \frac{a}{c}\right)= & (-1)^{\ell} 2^{2 \ell-d} \pi^{\frac{d}{2}+d \frac{\alpha}{2}-d s}|N c|^{-\frac{1}{2}+s-\frac{\alpha}{2}} \lambda_{-\underline{\nu}}(c) \times \\
& \times \prod_{j=1}^{d} \Gamma\left(\frac{1}{2}\left(s+l_{j}-i \nu_{j}\right)\right) \Gamma\left(\frac{1}{2}\left(s+l_{j}-\alpha-i \nu_{j}\right)\right) D_{\ell}\left(s, \alpha ; \underline{\nu} ; \frac{a}{c}\right) .
\end{aligned}
$$

We split the integral $I$ in 3.2 .20 into two parts, the one with $N \underline{y} \geq 1$ is called $I^{+}$and the other with $N \underline{y} \leq 1$ is denoted by $I^{-}$. The part $I^{+}$is entire in $s$ which follows from the decay properties of $K_{\mu}$. 
Assume Re $s$ sufficiently large. Then we may use (3.2.18) and 3.2.19) to obtain

$$
\begin{aligned}
I^{-}\left(s, \alpha ; \underline{\nu} ; \frac{a}{c}\right)= & (-1)^{\ell} I^{+}\left(1-s,-\alpha ;-\underline{\nu} ; \frac{-a^{*}}{c}\right) \\
+\frac{\delta_{\ell, 0} \delta_{\underline{\nu}, \underline{0}}}{\operatorname{vol}\left(\Lambda_{c}^{d-1}\right)}( & \pi^{-d \frac{\alpha}{2}}\left|D_{K}\right|^{\frac{\alpha}{2}-\frac{1}{4}}|N c|^{\frac{1}{2}(\alpha-1)} \Gamma\left(\frac{1}{2}(1+\alpha)\right)^{d} \zeta_{K}(1+\alpha)\left(\frac{1}{s-\alpha-1}-\frac{1}{s}\right) \\
& \left.\quad+\pi^{d \frac{\alpha}{2}}\left|D_{K}\right|^{-\frac{\alpha}{2}+\frac{1}{4}}|N c|^{\frac{1}{2}(\alpha+1)} \Gamma\left(\frac{1}{2}(1-\alpha)\right)^{d} \zeta_{K}(1-\alpha)\left(\frac{1}{s-1}-\frac{1}{s-\alpha}\right)\right) .
\end{aligned}
$$

Thus we have

$$
I\left(s, \alpha ; \underline{\nu} ; \frac{a}{c}\right)=(-1)^{\ell} I\left(1-s,-\alpha ;-\underline{\nu} ; \frac{-a^{*}}{c}\right) .
$$

Hence (3.2.21) shows that $D_{\ell}$ is meromorphic on $\mathbb{C}$ and satisfies the functional equation claimed in the lemma.

Now, we return to $\mathcal{D}_{m}$ given in (3.2.14), assuming that (3.2.17) holds. Let $v$ be as in (3.2.14). We define $Y_{\ell}\left(m ; \alpha, \frac{a}{c} ; h\right)$ as

$$
\frac{\operatorname{vol}\left(\Lambda_{c}^{d-1}\right)}{2 \pi i} \sum_{\underline{\tilde{\nu}} \in \Lambda_{c}^{d-1}} \lambda_{-\underline{\tilde{\nu}}}(m) \int_{(v)}|N(m)|^{\widetilde{h}_{\ell}}\left(s-i \tilde{\nu}_{1}, \ldots, s-i \tilde{\nu}_{d-1}, s+i \operatorname{Tr}(\underline{\tilde{\nu}})\right) D_{\ell}\left(s, \alpha ; \underline{\tilde{\nu}} ; \frac{a}{c}\right) d s,
$$

assuming $0<v-1-\max \{0, \operatorname{Re} \alpha\}<C_{0}$. Thus we have

$$
\mathcal{D}_{m}\left(\alpha, \frac{a}{c} ; h\right)=\sum_{\ell} Y_{\ell}\left(m ; \alpha, \frac{a}{c} ; h\right) .
$$

Moving the contour in $\int_{(v)}$ to the left, by Lemma 3.2 .1 we have

$$
\begin{aligned}
Y_{\ell}\left(m ; \alpha, \frac{a}{c} ; h\right)= & 2^{d} \delta_{\ell, \underline{0}}\left|D_{K}\right|^{-\frac{\alpha}{2}+\frac{1}{4}}|N c|^{\alpha-1}|N m| \widetilde{h}_{\ell}(\underline{1}) \zeta_{K}(1-\alpha) \\
& +2^{d} \delta_{\ell, \underline{0}}\left|D_{K}\right|^{\frac{\alpha}{2}-\frac{1}{4}}|N c|^{-\alpha-1}|N m|^{1+\alpha} \widetilde{h}_{\ell}(\underline{1+\alpha}) \zeta_{K}(1+\alpha) \\
& +\frac{|N c|^{\alpha+1} \operatorname{vol}\left(\Lambda_{c}^{d-1}\right)}{2 \pi i} \frac{(2 \pi)^{-d(\alpha+1)}}{\pi^{d}} \sum_{n \in \mathfrak{o}^{*} / \mathrm{U}_{c}} \frac{\sigma_{-\alpha}(n)}{|N n|} \operatorname{sgn}^{\ell}[n] e\left[\frac{a^{*} n}{c}\right] \sum_{\underline{\tilde{\nu}} \in \Lambda_{c}^{d-1}} \lambda_{-\underline{\underline{\nu}}}\left(\frac{m n}{c^{2}}\right) \times \\
& \times \int_{(b)} \Gamma_{\ell}(s-i \underline{\tilde{\nu}} ; \alpha)\left(\frac{(2 \pi)^{d} \sqrt{|N(m n)|}}{|N c|}\right)^{2 s} \widetilde{h}_{\ell}(s-i \underline{\tilde{u}}) d s
\end{aligned}
$$

with

$$
b<\min \{0,-\operatorname{Re} \alpha\} .
$$

The function $D_{\ell}$ is bounded for $\operatorname{Re} s>1+\max \{0, \operatorname{Re} \alpha\}$. Thus, by the functional equation we get

$$
D_{\ell}\left(s, \alpha ; \nu ; \frac{a}{c}\right) \ll(1+|s|)^{-2 \operatorname{Re} s+\operatorname{Re} \alpha}\left(\frac{2 \pi}{\sqrt{D_{K}|N c|}}\right)^{4 \operatorname{Re} s-2 \operatorname{Re} \alpha-2} \quad \text { for } \operatorname{Re} s<\min \{0,-\operatorname{Re} \alpha\} .
$$

Hence, if $C_{0}$ is sufficiently large in terms of $|\operatorname{Re} \alpha|$ and $|\beta|$, the resulting integral is absolutely convergent. We denote the integral $\int_{(b)}$ by $L(\underline{\tilde{v}})$ and observe by Stirling's formula and 3.2.17) that it is bounded by $(1+|\operatorname{Im} s|+\operatorname{Tr}(|\underline{\tilde{u}}|))^{-C_{0}}$. 
Using Poisson's summation formula we have

$$
\begin{aligned}
& \sum_{n \in \mathfrak{o}^{*} / \mathrm{U}_{c}} \frac{\sigma_{-\alpha}(n)}{|N n|} \operatorname{sgn}^{\ell}[n] e\left[\frac{a^{*} n}{c}\right] \sum_{\underline{\underline{\tilde{\nu}} \in \Lambda_{c}^{d-1}}} \lambda_{-\underline{\tilde{\nu}}}\left(\frac{m n}{c^{2}}\right) L(\underline{\tilde{\nu}}) \\
= & \operatorname{vol}\left(\Lambda_{c}^{d-1}\right)^{-1} \sum_{n \in \mathfrak{o}^{*} / \mathrm{U}_{c}} \frac{\sigma_{-\alpha}(n)}{|N n|} \operatorname{sgn}^{\ell}[n] e\left[\frac{a^{*} n}{c}\right] \sum_{\varepsilon \in \mathrm{U}_{c}} \int_{\mathbb{R}^{d-1}} \ldots \int L(\underline{\tau}) \lambda_{-\underline{\tau}}\left(\frac{\varepsilon m n}{c^{2}}\right) d \underline{\tau} \\
= & \operatorname{vol}\left(\Lambda_{c}^{d-1}\right)^{-1} \sum_{n \in \mathfrak{o}^{*}} \frac{\sigma_{-\alpha}(n)}{|N n|} \operatorname{sgn}^{\ell}[n] e\left[\frac{a^{*} n}{c}\right] \int \ldots \int L(\underline{\tau}) \lambda_{-\underline{\tau}}\left(\frac{m n}{c^{2}}\right) d \underline{\tau} .
\end{aligned}
$$

We reinsert the definition of $L(\underline{\tau})$ and have $(d-1)$ integrals over $\mathbb{R}$ and one integral over $(b)$. Then we substitute $s-i \tau_{j} \mapsto s_{j}$ for $j=1, \ldots, d-1$ and $s+i \operatorname{Tr}(\underline{\tau}) \mapsto s_{d}$, and put

$$
x(m, n ; c)=\left(\frac{4 \pi \sqrt{\left|m_{j} n_{j}\right|}}{\left|c_{j}\right|}\right)_{j=1}^{d} .
$$

We get

$$
\int \ldots \int L(\underline{\tau}) \lambda_{-\underline{\tau}}\left(\frac{m n}{c^{2}}\right) d \underline{\tau}=\frac{1}{d i^{d-1}} \int_{(b)} \ldots \int_{(b)} \Gamma_{\ell}(\underline{s} ; \alpha) \widetilde{h}_{\ell}(\underline{s}) \prod_{j=1}^{d}\left|\frac{x(m, n ; c) j}{2}\right|^{2 s_{j}} d \underline{s} .
$$

We define

$$
[h]_{\ell}(x ; \alpha):=\frac{1}{d i^{d-1}} \int_{(b) \cdots(b)} \ldots \int_{\ell} \Gamma_{\ell}(\underline{s} ; \alpha) \widetilde{h}_{\ell}(\underline{s}) \prod_{j=1}^{d}\left|\frac{x_{j}}{2}\right|^{2 s_{j}} d \underline{s} .
$$

By the same arguments as above for $L(\underline{\tau})$, we obtain convergence in a region which will be given in 3.2.27) and 3.2.28). Inserting 3.2.24 and 3.2.25 in 3.2.23) and 3.2.22 we have

$$
\begin{aligned}
\mathcal{D}_{m}\left(\alpha, \frac{a}{c} ; h\right)=\sum_{\ell}[ & 2^{d} \delta_{\ell, \underline{0}}\left|D_{K}\right|^{-\frac{\alpha}{2}+\frac{1}{4}}|N c|^{\alpha-1}|N m| \widetilde{h}_{\ell}(\underline{1}) \zeta_{K}(1-\alpha) \\
& +2^{d} \delta_{\ell, \underline{0}}\left|D_{K}\right|^{\frac{\alpha}{2}-\frac{1}{4}}|N c|^{-\alpha-1}|N m|^{1+\alpha} \widetilde{h}_{\ell}(\underline{1+\alpha}) \zeta_{K}(1+\alpha) \\
& \left.+\frac{|N c|^{\alpha+1}}{2 \pi i} \frac{(2 \pi)^{-d(\alpha+1)}}{\pi^{d}} \sum_{n \in \mathfrak{o}^{*}} \frac{\sigma_{-\alpha}(n)}{|N n|} \operatorname{sgn}^{\ell}[n] e\left[\frac{a^{*} n}{c}\right][h]_{\ell}(x(m, n ; c) ; \alpha)\right]
\end{aligned}
$$

Together with 3.2 .9 , we get

$$
\begin{aligned}
\mathcal{B}_{m}(\alpha, \beta ; h)=\sum_{\ell}[ & 2^{d} \delta_{\ell, \underline{0}}\left|D_{K}\right|^{-\frac{\alpha}{2}+\frac{1}{4}}|N m| \widetilde{h}_{\ell}(\underline{1}) \frac{\zeta_{K}(1-\alpha) \zeta_{K}(1-\beta)}{\zeta_{K}(2-\alpha-\beta)} \sigma_{\alpha+\beta-1}(m) \\
& +2^{d} \delta_{\ell, \underline{0}}\left|D_{K}\right|^{\frac{\alpha}{2}-\frac{1}{4}}|N m|^{1+\alpha} \widetilde{h}_{\ell}(\underline{1+\alpha}) \frac{\zeta_{K}(1+\alpha) \zeta_{K}(1-\beta)}{\zeta_{K}(2+\alpha-\beta)} \sigma_{-\alpha+\beta-1}(m) \\
& +\frac{\zeta_{K}(1-\beta)}{2 \pi i} \frac{(2 \pi)^{-d(\alpha+1)}}{\pi^{d}} \sum_{n \in \mathfrak{o}^{*}} \frac{\sigma_{-\alpha}(n)}{|N n|} \operatorname{sgn}^{\ell}[n] \times \\
& \left.\times \sum_{c \in \mathfrak{o}^{*}} \Psi(c)|N c|^{\alpha+\beta} S_{K}(m, n ; c)[h]_{\ell}(x(m, n ; c) ; \alpha)\right]
\end{aligned}
$$

where the Kloosterman sum $S_{K}(m, n ; c)$ over $K$ is given in 2.0.2). 
We assume

$$
|\alpha|,|\beta|<C_{1}
$$

for a suitable $C_{1}$, and we also assume that $C_{0}$ in 3.2 .17 is sufficiently large in terms of $C_{1}$. Then, with this and (3.2.17), the right hand side of 3.2 .26 ) converges absolutely for

$$
\operatorname{Re} \alpha+\operatorname{Re} \beta-2 b<-2 .
$$

In particular, by a shift of the contour we have the bound

$$
[h]_{\ell}(\underline{x} ; \alpha) \ll|N \underline{x}|^{2 b}(\operatorname{Tr}(1+|\underline{x}|))^{-\mu},
$$

for a $\mu>0$ and $b$ the integral contour from above. Furthermore alle the derivatives of $[h]_{\ell}$ are of rapid decay.

Later on, we shall spectrally decompose the sum over $c$ in 3.2 .26 . To this end we rearrange the sum over $c$ using the definition of $\Psi$. We have

$$
\begin{aligned}
& \sum_{c \in \mathfrak{o}^{*}} \Psi(c)|N c|^{\alpha+\beta} S_{K}(m, n ; c)[h]_{\ell}(x(m, n ; c) ; \alpha) \\
= & \frac{1}{(2 \pi)^{d-1}} \int_{\mathbb{R}^{d-1}} \ldots \int \widehat{\mathrm{p}}(\underline{\xi}) \sum_{c \in \mathfrak{o}^{*}}|N c|^{\alpha+\beta} \lambda_{\underline{\xi}}(c) S_{K}(m, n ; c)[h]_{\ell}(x(m, n ; c) ; \alpha) d \underline{\xi} \\
= & \frac{1}{(2 \pi)^{d-1}}\left((2 \pi)^{d} \sqrt{|N(m n)|}\right)^{\alpha+\beta+1} \int_{\mathbb{R}^{d-1}} \ldots \int \widehat{\mathrm{p}}(\underline{\xi}) \lambda_{\underline{\xi}}(m n) S_{m, n}\left(\alpha, \beta, \xi ;[h]_{\ell}\right) d \underline{\xi}
\end{aligned}
$$

where

$$
S_{m, n}\left(\alpha, \beta, \xi ;[h]_{\ell}\right)=\sum_{c \in \mathfrak{o}^{*}}|N c|^{-1} S_{K}(m, n ; c)[[h]]_{\ell}(x(m, n ; c) ; \alpha, \beta ; \xi)
$$

with

$$
[[h]]_{\ell}(x ; \alpha, \beta ; \xi)=\left|\frac{N x}{2^{d}}\right|^{-(\alpha+\beta)-1} \lambda_{-\underline{\xi}}(x)[h]_{\ell}(x ; \alpha) .
$$

For $\alpha$ and $\beta$ satisfying the conditions 3.2.17) and (3.2.27), by shifting the contour we obtain the estimate

$$
[[h]]_{\ell}(x ; \alpha, \beta ; \xi) \ll(\operatorname{Tr}(1+|\underline{x}|))^{-\mu} \cdot \begin{cases}|N x|^{-\frac{1}{2} C_{0}} & \text { if }|N x| \geq 1 \\ |N x| & \text { if }|N x|<1\end{cases}
$$

with $\mu>0$ as in the bound of $[h]_{\ell}$. Furthermore the derivatives of $[[h]]_{\ell}$ are of rapid decay if at least one variable tends to $0+$ or $+\infty$.

\subsubsection{Spectral Decomposition of $\mathcal{B}_{m}$}

The sum $S_{m, n}\left(\alpha, \beta, \xi ;[h]_{\ell}\right)$ as defined in 3.2 .31 has the right form to apply the geometric sum formula given in Lemma 3.1.5. Recall that $m \succ 0$ and thus $\eta_{V}(m)=\eta_{V}(1)$.

The definitions of $[h]_{\ell}$ and $[[h]]_{\ell}$ imply that $[[h]]_{\ell}(x ; \alpha, \beta, \xi)$ and its derivatives are smooth and by 3.2.33 of rapid decay if at least one variable tends to $0+$ or $+\infty$. Therefore Lemma 3.1.5 can safely 
be applied to $S_{m, n}\left(\alpha, \beta, \xi ;[h]_{\ell}\right)$, provided

$$
|\operatorname{Re} \alpha|+\operatorname{Re} \beta<-\frac{1}{2} C_{1}
$$

For instance choose $\operatorname{Re} \beta$ negative and large, and $|\operatorname{Re} \alpha|$ relatively small.

Lemma 3.1 .5 yields the decomposition

$$
\begin{aligned}
S_{m, n}\left(\alpha, \beta, \xi ;[h]_{\ell}\right)= & \sum_{V} a_{V} \eta_{V}(1) t_{V}((m)) \eta_{V}(n) t_{V}((n)) \mathrm{B}_{[n]}[[h]]_{\ell}\left(\kappa_{V} ; \alpha, \beta, \xi\right) \\
& +C_{e} \sum_{\underline{\nu} \in \Lambda^{d-1}} \lambda_{-\underline{\nu}}(m n) \int_{-\infty}^{\infty} \frac{\sigma_{2 i t}(m, 2 \underline{\nu}) \sigma_{2 i t}(n, 2 \underline{\nu})}{|N(m n)| i t\left|\zeta_{K}(1+2 i t, 2 \underline{\nu})\right|^{2}} \mathrm{~B}_{[n]}[[h]]_{\ell}(t+\underline{\nu} ; \alpha, \beta, \xi) d t \\
= & :\left\{\mathrm{S}_{m, n}^{c}+\mathrm{S}_{m, n}^{e}\right\}\left(\alpha, \beta, \xi ;[h]_{\ell}\right)
\end{aligned}
$$

where $t+\underline{\nu}=\left(t+\nu_{1}, \ldots, t+\nu_{d-1}, t-\operatorname{Tr}(\underline{\nu})\right)$.

The uniformity of the convergence follows by inserting the bounds for all involved parameters stated in the previous sections. More precisely, we use the estimate (3.1.44) to bound the functions $\mathrm{B}_{[n]}[[h]]_{\ell}$. Then Lemma 3.1.4 gives the convergence for the first term, and Lemma 3.1.1 combined with trivial estimates for the divisor functions does it for the second term.

We insert the decomposition into (3.2.30) and (3.2.26), and factorize $n \in \mathfrak{o}^{*}$ into $n \cdot \varepsilon$ where $\varepsilon$ runs over the units, and $n \in \mathfrak{o}^{*} \bmod P(\epsilon)^{2}$.

Then the contribution of $S_{m, n}^{c}$ equals

$$
\begin{aligned}
& \frac{(2 \pi)^{d \beta}}{i\left(2 \pi^{2}\right)^{d}} \zeta_{K}(1-\beta)(N m)^{\frac{1}{2}(\alpha+\beta+1)} \times \\
\times & \sum_{n \in \mathfrak{o}^{*} \bmod P(\epsilon)^{2}} \frac{\sigma_{-\alpha}(n)}{(N n)^{\frac{1}{2}(1-\alpha-\beta)}} \operatorname{sgn}^{\ell}[n] \sum_{V} a_{V} \eta_{V}(1) t_{V}((m)) \eta_{V}(n) t_{V}((n)) \times \\
\times & \sum_{\underline{\nu} \in \mathbb{Z}^{d-1}} \int \ldots \int e^{i \operatorname{Tr}(\underline{\nu} \underline{\xi})} \widehat{\mathrm{p}}(\underline{\xi}) \lambda_{\underline{\xi}}(m n) \mathrm{B}_{[n]}[[h]]_{\ell}\left(\kappa_{V} ; \alpha, \beta ; \underline{\xi}\right) d \underline{\xi} .
\end{aligned}
$$

The function $\mathrm{B}_{[n]}[[h]]_{\ell}$ is smooth in $\xi$ and is bounded by (3.1.44) with $f=[[h]]_{\ell}$ and $C_{f}=C_{1}$. Thus the integral decays sufficiently fast in $\nu$ and $\kappa_{V}$, and the triple sum converges absolutely. Using Poisson's summation formula, we see that only the term with $\underline{\xi}=\underline{\nu}=0$ survives. Hence the expression changes to

$$
\begin{aligned}
& \frac{(2 \pi)^{d \beta}}{i\left(2 \pi^{2}\right)^{d}} \zeta_{K}(1-\beta)(N m)^{\frac{1}{2}(\alpha+\beta+1)} \sum_{n \in \mathfrak{o}^{*} \bmod P(\epsilon)^{2}} \frac{\sigma_{-\alpha}(n)}{(N n)^{\frac{1}{2}(1-\alpha-\beta)}} \operatorname{sgn}^{\ell}[n] \times \\
\times & \sum_{V} a_{V} \eta_{V}(1) t_{V}((m)) \eta_{V}(n) t_{V}((n)) \frac{1}{2^{d-1}} \mathrm{~B}_{[n]}[[h]]_{\ell}\left(\kappa_{V} ; \alpha, \beta ; 0\right) .
\end{aligned}
$$

The factors $\operatorname{sgn}[n]$ and $\eta_{V}(n)$ depend only on the sign of $n$, in other words they depend on the unit $\varepsilon$ such that $n=n^{\prime} \varepsilon$ with $n^{\prime} \succ 0$. Thus we can rewrite the sum over $n$ as

$$
\begin{aligned}
& \frac{2(2 \pi)^{d \beta}}{i(2 \pi)^{2 d}} \zeta_{K}(1-\beta)(N m)^{\frac{1}{2}(\alpha+\beta+1)} \sum_{V} a_{V} \eta_{V}(1) t_{V}((m)) \times \\
\times & \sum_{\varepsilon \bmod P(\epsilon)^{2}} \operatorname{sgn}^{\ell}[\varepsilon] \eta_{V}(\varepsilon) \mathrm{B}_{[\varepsilon]}[[h]]_{\ell}\left(\kappa_{V} ; \alpha, \beta ; 0\right) \cdot \sum_{\mathfrak{n}} t_{V}(\mathfrak{n}) \frac{\sigma_{-\alpha}(\mathfrak{n})}{(N \mathfrak{n})^{\frac{1}{2}(1-\alpha-\beta)}} .
\end{aligned}
$$


Then the sum over $\mathfrak{n}$ equals $H_{V}\left(\frac{1}{2}(1-\alpha-\beta)\right) H_{V}\left(\frac{1}{2}(1+\alpha-\beta)\right)$. We denote the sum over $\varepsilon$ by $\mathrm{B}^{V, \ell}$. Hence we have

$$
S_{m, n}^{c}=\frac{2(2 \pi)^{d \beta}}{i(2 \pi)^{2 d}}(N m)^{\frac{1}{2}(\alpha+\beta+1)} \sum_{V} a_{V} \eta_{V}(1) t_{V}((m)) H_{V}\left(\frac{1}{2}(1-\alpha-\beta)\right) H_{V}\left(\frac{1}{2}(1+\alpha-\beta)\right) \mathrm{B}^{V, \ell} .
$$

This sum converges absolutely by Lemma 3.1.6 and 3.1.44.

The contribution coming from $S_{m, n}^{e}$ can be treated in a similar way. We get

$$
\begin{aligned}
& \frac{2(2 \pi)^{d \beta}}{i(2 \pi)^{2 d}} C_{e}(N m)^{\frac{1}{2}(\alpha+\beta+1)} \sum_{\underline{\nu} \in \Lambda^{d-1}} \lambda_{-\underline{\nu}}(m) \int_{-\infty}^{\infty} \frac{\sigma_{2 i t}(m, 2 \underline{\nu})}{(N m)^{i t}\left|\zeta_{K}(1+2 i t, 2 \underline{\nu})\right|^{2}} \times \\
\times & \zeta_{K}\left(\frac{1}{2}(1+\alpha-\beta)-i t, \underline{\nu}\right) \zeta_{K}\left(\frac{1}{2}(1+\alpha-\beta)+i t,-\underline{\nu}\right) \zeta_{K}\left(\frac{1}{2}(1-\alpha-\beta)-i t, \underline{\nu}\right) \times \\
\times & \zeta_{K}\left(\frac{1}{2}(1-\alpha-\beta)+i t,-\underline{\nu}\right) \mathrm{B}^{\varepsilon_{\underline{\nu}}, \ell}[[h]]_{\ell}(t+\underline{\nu} ; \alpha, \beta, 0) d t
\end{aligned}
$$

where $t+\underline{\nu}=\left(t+\nu_{1}, \ldots, t+\nu_{d-1}, t-\operatorname{Tr}(\underline{\nu})\right)$. Lemma 3.1.1. 3.1.44) and trivial estimates for the factors $\zeta_{K}(\sigma+i t, \pm \underline{\nu})$, as $\sigma>1$, show the convergence.

Thus we have a spectral decomposition for $\mathcal{B}_{m}$, as long as $\alpha$ and $\beta$ satisfy our assumptions (3.2.17), (3.2.27) and 3.2.34). We have

$$
\begin{aligned}
\mathcal{B}_{m}(\alpha, \beta ; h)= & 2^{d}\left|D_{K}\right|^{-\frac{\alpha}{2}+\frac{1}{4}}|N m| \widetilde{h}_{\underline{0}}(\underline{1}) \frac{\zeta_{K}(1-\alpha) \zeta_{K}(1-\beta)}{\zeta_{K}(2-\alpha-\beta)} \sigma_{\alpha+\beta-1}(m) \\
+ & 2^{d}\left|D_{K}\right|^{\frac{\alpha}{2}-\frac{1}{4}}|N m|^{1+\alpha} \widetilde{h}_{\underline{0}}(\underline{1+\alpha}) \frac{\zeta_{K}(1+\alpha) \zeta_{K}(1-\beta)}{\zeta_{K}(2+\alpha-\beta)} \sigma_{-\alpha+\beta-1}(m) \\
+\sum_{\ell}[ & \frac{2(2 \pi)^{d \beta}}{i(2 \pi)^{2 d}}(N m)^{\frac{1}{2}(\alpha+\beta+1)} \sum_{V} a_{V} \eta_{V}(1) t_{V}((m)) H_{V}\left(\frac{1}{2}(1-\alpha-\beta)\right) H_{V}\left(\frac{1}{2}(1+\alpha-\beta)\right) \mathrm{B}^{V, \ell} \\
& +\frac{2(2 \pi)^{d \beta}}{i(2 \pi)^{2 d}} C_{e}(N m)^{\frac{1}{2}(\alpha+\beta+1)} \sum_{\underline{\nu} \in \Lambda^{d-1}} \lambda_{-\underline{\nu}}(m) \int_{-\infty}^{\infty} \frac{\sigma_{2 i t}(m, 2 \underline{\nu})}{(N m)^{i t}\left|\zeta_{K}(1+2 i t, 2 \underline{\nu})\right|^{2}} \times \\
& \times \zeta_{K}\left(\frac{1}{2}(1+\alpha-\beta)-i t, \underline{\nu}\right) \zeta_{K}\left(\frac{1}{2}(1+\alpha-\beta)+i t,-\underline{\nu}\right) \zeta_{K}\left(\frac{1}{2}(1-\alpha-\beta)-i t, \underline{\nu}\right) \times \\
& \left.\times \zeta_{K}\left(\frac{1}{2}(1-\alpha-\beta)+i t,-\underline{\nu}\right) \mathrm{B}^{\varepsilon_{\underline{\nu}}, \ell}[[h]]_{\ell}(t+\underline{\nu} ; \alpha, \beta, 0) d t\right] .
\end{aligned}
$$

But the domain coming from these assumptions does not contain the point $(\alpha, \beta)=(0,0)$ we need for the application to $\mathcal{Z}_{2}(g, \omega ; K)$. Thus, we have to continue the decomposition to a neighborhood of $(\alpha, \beta)=(0,0)$. To this end we consider the transform of $h$ given by

$$
\Phi_{*}(\underline{r} ; \alpha, \beta ; h)=\frac{1}{i} \sum_{\ell} \sum_{\varepsilon \bmod P(\epsilon)^{2}} \operatorname{sgn}^{\ell}[\varepsilon] \eta_{*}(\varepsilon) \mathrm{B}_{[\varepsilon]}[[h]]_{\ell}(\underline{r} ; \alpha, \beta, 0)
$$

where $*=V$ or $\mathcal{E}_{\underline{\nu}}$ and $\underline{r}=\kappa_{V}$ or $t+\underline{\nu}=\left(t+\nu_{1}, \ldots, t+\nu_{d-1}, t-\operatorname{Tr}(\underline{\nu})\right)$ respectively.

We use the definitions of $[[h]]_{\ell}$ in (3.2.32), $[h]_{\ell}$ in 3.2.25), $\widetilde{h}_{\ell}$ in 3.2.15) and $\Gamma_{\ell}$ in Lemma 3.2.1. 
We obtain

$$
\begin{aligned}
\sum_{\ell} \operatorname{sgn}^{\ell}[\varepsilon][[h]]_{\ell}(x ; \alpha, \beta, 0) & =\frac{1}{2^{d} d i^{d-1}}\left|\frac{N x}{2^{d}}\right|^{1-\alpha-\beta} \times \\
\times \sum_{\mathrm{e} \in\{ \pm 1\}^{d}} \int_{(b)} \ldots \int_{(b)} & \prod_{j=1}^{d}\left[\left[\left(1+e_{j} \operatorname{sgn}\left(\varepsilon_{j}\right)\right) \cos \left(\frac{\pi}{2} \alpha\right)-\left(1-e_{j} \operatorname{sgn}\left(\varepsilon_{j}\right)\right) \cos \left(\pi\left(s_{j}-\frac{1}{2} \alpha\right)\right)\right] \times\right. \\
& \left.\times \Gamma\left(1-s_{j}\right) \Gamma\left(1+\alpha-s_{j}\right)\right] \widetilde{h}(\underline{s} ; \mathrm{e})\left|\frac{x_{1}}{2}\right|^{2 s_{1}} \ldots\left|\frac{x_{d}}{2}\right|^{2 s_{d}} d \underline{s}
\end{aligned}
$$

where $b$ is the contour from above, and $\varepsilon$ is a unit. Stirling's formula and 3.2 .17 imply that the integral converges absolutely.

Now we apply $\mathrm{B}_{\mathrm{e}}$ with respect to $x$ to this expression, and use the equations 3.1.45 - 3.1.47) to get a new expression for $\Phi_{*}$, namely

$$
\begin{aligned}
\Phi_{*}(\underline{r} ; \alpha, \beta ; h) & =\frac{1}{(2 i)^{d} d} \sum_{\mathrm{e} \in\{ \pm 1\}^{d}} \sum_{\varepsilon \bmod P(\epsilon)^{2}} \eta_{*}(\varepsilon) \int \ldots \int_{(-i \infty, i \infty)^{d}} \widetilde{h}(\underline{s} ; \mathrm{e}) \prod_{j=1}^{d}\left[\Delta_{e_{j}}^{\operatorname{sgn}\left(\varepsilon_{j}\right)}\left(s_{j}, r_{j} ; \alpha, \beta\right) \times\right. \\
& \left.\times \Gamma\left(s_{j}-\frac{1}{2}(1+\alpha+\beta)-i r_{j}\right) \Gamma\left(s_{j}-\frac{1}{2}(1+\alpha+\beta)+i r_{j}\right) \Gamma\left(1-s_{j}\right) \Gamma\left(1+\alpha-s_{j}\right)\right] d \underline{s}
\end{aligned}
$$

where

$$
\begin{aligned}
\Delta_{e_{j}}^{ \pm} & =\left\{\left(1 \pm e_{j}\right) \cos \left(\frac{\pi}{2} \alpha\right)-\left(1 \mp e_{j}\right) \cos \left(\pi\left(s_{j}-\frac{1}{2} \alpha\right)\right)\right\} \times \\
& \times\left\{(1 \mp 1) \cosh \left(\pi r_{j}\right)+(1 \pm 1) \sin \left(\pi\left(s_{j}-\frac{1}{2}(\alpha+\beta)\right)\right)\right\} .
\end{aligned}
$$

The $s_{j}$-contour separates the poles of $\Delta_{e_{j}}^{\operatorname{sgn}\left(\epsilon_{j}\right)}\left(s_{j}, r_{j} ; \alpha, \beta\right) \Gamma\left(s_{j}-\frac{1}{2}(\alpha+\beta+1)-i r_{j}\right) \Gamma\left(s_{j}-\frac{1}{2}(\alpha+\beta+1)+i r_{j}\right)$ and those of $\Gamma\left(1-s_{j}\right) \Gamma\left(1+\alpha-s_{j}\right)$, and it is assumed that the contour can be drawn, i.e. if we would pass through a pole, we choose a small semicircle around it. Under the assumption (3.2.34) one may use the contour $\operatorname{Re} s_{j}=b$, with the bounds $\frac{1}{2}(\operatorname{Re} \alpha+\operatorname{Re} \beta+1)<b<\min \{0,-\operatorname{Re} \alpha\}$. Then we move the contour appropriately to get 3.2.36). As the right hand side exists on a larger domain, the representation 3.2.36) gives an analytic continuation of $\Phi_{*}$.

In the next steps, we reformulate a factor of $\Phi_{*}$ and state some estimates for it.

Consider the expression

$$
\sum_{\varepsilon \bmod P(\epsilon)^{2}} \eta_{*}(\varepsilon) \prod_{j=1}^{d} \Delta_{e_{j}}^{\mathrm{sgn}\left(\varepsilon_{j}\right)}
$$

As in BM01, (5.12)-(5.13)] one has to consider the possible values of $*=V$ and $q_{V}$ and for $*=\mathcal{E}_{\nu}$ separately, and reformulate the expression in every case. Combining these results we obtain for any space $*=V$ and $\mathcal{E}_{\nu}$ with $\eta_{*}(1) \neq 0$ the equality

$$
\sum_{\varepsilon \bmod P(\epsilon)^{2}} \eta_{*}(\varepsilon) \prod_{j=1}^{d} \Delta_{e_{j}}^{\mathrm{sgn}\left(\varepsilon_{j}\right)}=\eta_{*}(1) \prod_{j=1}^{d}\left(\Delta_{e_{j}}^{+}+\lambda_{*}\left(\varepsilon_{j}\right) \Delta_{e_{j}}^{-}\right)
$$

where $\varepsilon_{j}$ on the right hand side is as in Lemma 3.1.6. 


\section{(3.2.2) Lemma}

Assume that (3.2.17) and (3.2.27) hold for $\alpha, \beta$ and $h$, and let $V$, the eigenvalue $\kappa_{V}=\left(\kappa_{1}, \ldots, \kappa_{d}\right)$, and $q_{V}=\left(q_{1}, \ldots, q_{d}\right)$ be as in 3.1.28) and Lemma 3.1.3. Then $\Phi_{V}\left(\kappa_{V} ; \alpha, \beta ; h\right)$ is regular in $\kappa_{V}$ and bounded by

$$
\Phi_{V}\left(\kappa_{V} ; \alpha, \beta ; h\right) \ll\left(1+\left|\kappa_{1}\right|+\cdots+\left|\kappa_{d}\right|\right)^{-\frac{C_{0}}{2}}
$$

uniformly in $V$ and $\alpha, \beta$, provided

$$
|\operatorname{Re} \alpha|+\operatorname{Re} \beta<2 \min _{j}\left\{\left|\operatorname{Im} \kappa_{j}+\frac{1}{2}\right|+\delta_{j}\right\}
$$

with

$$
\delta_{j}= \begin{cases}0, & \text { if } q_{j}=0 \\ 1, & \text { if } q_{j} \neq 0\end{cases}
$$

Likewise $\Phi_{\varepsilon_{\nu}}$ is regular in $t$ and $\underline{\nu}$. Let $t+\underline{\nu}=\left(t+\nu_{1}, \ldots, t+\nu_{d-1}, t-\operatorname{Tr}(\underline{\nu})\right)$. Then $\Phi_{\varepsilon_{\nu}}(t+\underline{\nu} ; \alpha, \beta ; h)$ is bounded by

$$
\Phi_{\varepsilon_{\nu}}(t+\underline{\nu} ; \alpha, \beta ; h) \ll\left(1+|t|+\left|\nu_{1}\right|+\cdots+\left|\nu_{d-1}\right|\right)^{-\frac{C_{0}}{2}},
$$

provided

$$
|\operatorname{Re} \alpha|+\operatorname{Re} \beta<1-2|\operatorname{Im} t| .
$$

Proof.

First we consider the case $q_{j} \neq 0$ for all $j$. Then $i \kappa_{j}=l_{j}-\frac{1}{2}$, and we modify the $\Gamma$-factors of 3.2 .36 using (3.1.48). We have

$$
\begin{aligned}
\Phi_{V}\left(\kappa_{V} ; \alpha, \beta ; h\right) & =\frac{(-1)^{l_{1}+\cdots+l_{d}-d}}{d} \sum_{\mathrm{e}} \sum_{\varepsilon} \eta_{V}(\varepsilon) \times \\
& \times \int_{(-i \infty, i \infty)^{d}} \ldots \int_{\widetilde{h}}(\underline{s} ; \mathrm{e}) \prod_{j=1}^{d}\left[\left[\left(1+e_{j} \operatorname{sgn}\left(\varepsilon_{j}\right)\right) \cos \left(\frac{\pi}{2} \alpha\right)-\left(1-e_{j} \operatorname{sgn}\left(\varepsilon_{j}\right)\right) \cos \left(\pi\left(s_{j}-\frac{1}{2} \alpha\right)\right)\right] \times\right. \\
& \left.\times \Gamma\left(1-s_{j}\right) \Gamma\left(1+\alpha-s_{j}\right) \frac{\Gamma\left(l_{j}-1+s_{j}-\frac{1}{2}(\alpha+\beta)\right)}{\Gamma\left(l_{j}+1-s_{j}+\frac{1}{2}(\alpha+\beta)\right)}\right] d \underline{s}
\end{aligned}
$$

where the contours can be drawn under the assumption 3.2.38). Thus, the regularity of $\Phi_{V}$ follows. To obtain the decay of $\Phi_{V}$ we shift the contours appropriately to the left, and use Stirling's formula and (3.2.17), as well as trivial estimates for the trigonometric functions.

If $q_{j}=0$ for some $j$, we do not need to rewrite the integral, and the shift of contour is not needed for the $j$-th integral. After reformulating the integrals with $q_{j} \neq 0$ the regularity follows and one proceeds as above with Stirling's formula to get the estimates on $\Phi_{*}$, under the assumption (3.2.38). For the spaces $\mathcal{E}_{\underline{\underline{\nu}}}$ the proof is analogously to $q_{j}=0$, under the assumption 3.2.40 .

Now we can state a first explicit formula for $\mathcal{B}_{m}$ in a neighborhood of $(\alpha, \beta)=(0,0)$ : 


\section{(3.2.3) Theorem}

Let $\mathcal{B}_{m}(\alpha, \beta ; h)$ be defined by (3.2.6), and assume (3.2.17). Let $\alpha, \beta$ be such that

$$
-1<\operatorname{Re}( \pm \alpha+\beta)<\frac{3}{5}
$$

Then we have the spectral decomposition

$$
\mathcal{B}_{m}(\alpha, \beta ; h)=\left\{\mathcal{B}_{m}^{(r)}+\mathcal{B}_{m}^{(c)}+\mathcal{B}_{m}^{(e)}\right\}(\alpha, \beta ; h)
$$

where

$$
\begin{aligned}
\mathcal{B}_{m}^{(r)}(\alpha, \beta ; h) & =\left|D_{K}\right|^{-\frac{\alpha}{2}+\frac{1}{4}} \frac{\zeta_{K}(1-\alpha) \zeta_{K}(1-\beta)}{\zeta_{K}(2-\alpha-\beta)}(N m) \sigma_{\alpha+\beta-1}(m) \ddot{h}(0,0) \\
& +\left|D_{K}\right|^{\frac{\alpha}{2}-\frac{1}{4}} \frac{\zeta_{K}(1+\alpha) \zeta_{K}(1-\beta)}{\zeta_{K}(2+\alpha-\beta)}(N m)^{1+\alpha} \sigma_{-\alpha+\beta-1}(m) \ddot{h}(\alpha, 0) \\
& +\frac{2^{2 d}}{d \pi^{d}} R_{K}\left|D_{K}\right|^{\beta+\frac{1}{2}} \frac{\zeta_{K}(1-\alpha) \zeta_{K}(1+\beta)}{\zeta_{K}(2-\alpha+\beta)}(N m)^{1+\beta} \sigma_{\alpha-\beta-1}(m) \ddot{h}(0, \beta) \\
& +\frac{2^{2 d}}{d \pi^{d}} R_{K}\left|D_{K}\right|^{\beta+\frac{1}{2}} \frac{\zeta_{K}(1+\alpha) \zeta_{K}(1+\beta)}{\zeta_{K}(2+\alpha+\beta)}(N m)^{1+\alpha+\beta} \sigma_{-\alpha-\beta-1}(m) \ddot{h}(\alpha, \beta), \\
\mathcal{B}_{m}^{(c)}(\alpha, \beta ; h) & =\frac{2(2 \pi)^{d \beta}}{(2 \pi)^{2 d}}(N m)^{\frac{1}{2}(\alpha+\beta+1)} \sum_{V} a_{V} \eta_{V}(1) t_{V}((m)) H_{V}\left(\frac{1}{2}(1-\alpha-\beta)\right) \\
& \times H_{V}\left(\frac{1}{2}(1+\alpha-\beta)\right) \Phi_{V}\left(\kappa_{V} ; \alpha, \beta ; h\right), \\
\mathcal{B}_{m}^{(e)}(\alpha, \beta ; h) & =\frac{2(2 \pi)^{d \beta}}{(2 \pi)^{2 d}} C_{e} \sum_{\underline{\nu} \in \Lambda^{d-1}} \lambda_{-\underline{\nu}}(m) \int_{-\infty}^{\infty} \frac{\sigma_{2 i t}(m, 2 \underline{\nu})}{(N m)^{i t}\left|\zeta_{K}(1+2 i t, 2 \underline{\nu})\right|^{2}} \times \\
\times & \zeta_{K}\left(\frac{1}{2}(1+\alpha-\beta)-i t, \underline{\nu}\right) \zeta_{K}\left(\frac{1}{2}(1+\alpha-\beta)+i t,-\underline{\nu}\right) \zeta_{K}\left(\frac{1}{2}(1-\alpha-\beta)-i t, \underline{\nu}\right) \times \\
& \times \zeta_{K}\left(\frac{1}{2}(1-\alpha-\beta)+i t,-\underline{\nu}\right) \Phi_{\varepsilon_{\nu}}(t+\underline{\nu} ; \alpha, \beta ; h) d t .
\end{aligned}
$$

Here $V, \kappa_{V}, t_{V}, \eta_{V}, a_{V}, H_{V}$ are as defined in Section 3.1.2, $\Phi_{*}$ is given by (3.2.36) and as before the vector $t+\underline{\nu}=\left(t+\nu_{1}, \ldots, t+\nu_{d-1}, t-\operatorname{Tr}(\underline{\nu})\right)$. The function $\ddot{h}$ is defined by

$$
\ddot{h}\left(\eta_{1}, \eta_{2}\right)=\int \ldots \int h(\underline{u})|N \underline{u}|^{\eta_{1}}\left(\left|1+u_{1}\right| \cdots\left|1+u_{d}\right|\right)^{\eta_{2}} d \underline{u} .
$$

Remark that $h(\underline{u})$ is of rapid decay if $u_{j}$ tends to infinity, 0 or -1 for at least one $j$. The expressions for $\mathcal{B}_{m}^{(r)}, \mathcal{B}_{m}^{(c)}$ and $\mathcal{B}_{m}^{(e)}$ are regular in the domain 3.2 .41 .

\section{Proof.}

The spectral decomposition holds in the domain (3.2.34). Thus we have to prove that all terms can be continued to the domain (3.2.41).

Consider the cuspidal contribution. The sum is absolutely and uniformly convergent in the domain

$$
|\operatorname{Re} \alpha|+\operatorname{Re} \beta<2 \min _{V} \min _{j}\left\{\left|\operatorname{Im} \kappa_{j}+\frac{1}{2}\right|+\delta_{j}\right\} \text {, by Lemma 3.1.6 and Lemma 3.2.2, }
$$

and it is regular there. By the possible values of $\kappa_{j}$ this domain contains the domain (3.2.41). Thus $\mathcal{B}_{m}^{(c)}$ is of the form stated in the Theorem. 
Now we consider the contribution of the Eisenstein series. In the domain (3.2.34 this is of the form stated in the Theorem, but the domain (3.2.41) is not contained in (3.2.34). Hence we have to extend the domain for the contribution of the Eisenstein series and pay attention to possible singularities.

However, for the terms with $\underline{\nu} \neq 0$ the integrand is regular in 3.2 .41 , and the sum $\sum_{\nu \in \Lambda^{d-1}}$ converges absolutely by Lemma 3.2 .2 .

Thus, we exclude this part from our consideration and restrict our attention to the term $\underline{\nu}=\underline{0}$, namely

$$
\begin{aligned}
& \frac{2(2 \pi)^{d \beta}}{(2 \pi)^{2 d}} C_{e} \int_{-\infty}^{\infty} \frac{\sigma_{2 i t}(m)}{(N m)^{i t}\left|\zeta_{K}(1+2 i t)\right|^{2}} \zeta_{K}\left(\frac{1}{2}(1+\alpha-\beta)-i t\right) \zeta_{K}\left(\frac{1}{2}(1+\alpha-\beta)+i t\right) \times \\
\times & \zeta_{K}\left(\frac{1}{2}(1-\alpha-\beta)-i t\right) \zeta_{K}\left(\frac{1}{2}(1-\alpha-\beta)+i t\right) \Psi_{t}(\alpha, \beta ; h) d t .
\end{aligned}
$$

Here $(\alpha, \beta)$ is in the domain 3.2 .34 , and

$$
\Psi_{t}(\alpha, \beta ; h)=\Phi_{\varepsilon_{0}}(\underbrace{t, \ldots, t}_{d-\text { times }} ; \alpha, \beta ; h)
$$

The integral is regular when $|\operatorname{Re} \alpha|+\operatorname{Re} \beta<-1$, as in this domain $\operatorname{Re}\left(\frac{1}{2}(1 \pm \alpha-\beta)\right)>1$. We consider the subdomain $-\frac{5}{4}<\operatorname{Re}( \pm \alpha+\beta)<-1$. We move the contour to $\operatorname{Im} t=\frac{1}{8}$ by noting (3.2.39) and 3.2 .40 . To avoid poles on the contour, we can choose an appropriate broken line instead of a horizontal line.

The resulting integral is regular for $-\frac{5}{4}<\operatorname{Re}( \pm \alpha+\beta)<-\frac{3}{4}$. Now we restrict this domain to

$$
-1<\operatorname{Re}( \pm \alpha+\beta)<-\frac{3}{4}
$$

and shift the $t$-contour back to $\operatorname{Im} t=0$.

As the new integral is regular in 3.2.41, $\mathcal{B}_{m}^{(e)}$ continues to 3.2.41.

The first shift of the contour gives us poles at $t=-\frac{1}{2}(1 \pm \alpha+\beta) i$, and those from the factor $\zeta_{K}^{-1}(1+2 i t)$. By the second one we encounter poles from $\zeta_{K}^{-1}(1+2 i t)$ and at the points $t=+\frac{1}{2}(1 \pm \alpha+\beta) i$.

The residual terms coming from $\zeta_{K}^{-1}$ cancel, and we are left with the terms coming from the residues at $t= \pm \frac{1}{2}(1 \pm \alpha+\beta) i$, given by

$$
\begin{aligned}
& \frac{(2 \pi)^{d \beta}}{\left(2 \pi^{2}\right)^{d}} \frac{R_{K}}{\sqrt{\left|D_{K}\right|}} C_{e} \cdot(N m)^{1+\beta} \sigma_{\alpha-\beta-1}(m) \frac{\zeta_{K}(-\beta) \zeta_{K}(1-\alpha)}{\zeta_{K}(2-\alpha+\beta)} \Psi_{\frac{1}{2}(1-\alpha+\beta) i}(\alpha, \beta ; h) \\
+ & \frac{(2 \pi)^{d \beta}}{\left(2 \pi^{2}\right)^{d}} \frac{R_{K}}{\sqrt{\left|D_{K}\right|}} C_{e} \cdot(N m)^{1+\alpha+\beta} \sigma_{-\alpha-\beta-1}(m) \frac{\zeta_{K}(-\beta) \zeta_{K}(1+\alpha)}{\zeta_{K}(2+\alpha+\beta)} \Psi_{\frac{1}{2}(1+\alpha+\beta) i}(\alpha, \beta ; h) .
\end{aligned}
$$

We used that $\Psi_{t}$ is an even function in $t$, and that the residue of $\zeta_{K}(s)$ at $s=1$ is equal to $\frac{2^{d-1} R_{K}}{\sqrt{\left|D_{K}\right|}}$. Hence, we are left with computing the factors $\Psi_{\frac{1}{2}(1 \mp \alpha+\beta) i}$.

Let $r_{j}=\frac{1}{2}(1-\alpha+\beta) i$. Then

$$
\sum_{\varepsilon \bmod P(\epsilon)^{2}} \eta_{*}(\varepsilon) \prod_{j=1}^{d} \Delta_{e_{j}}^{\mathrm{sgn}\left(\varepsilon_{j}\right)}\left(s_{j}, r_{;} \alpha, \beta\right)=2^{2 d} \prod_{j=1}^{d} e_{j}\left[\sin \left(\pi\left(s_{j}-\alpha\right)\right) \cos \left(\frac{1}{2} \pi\left(\left(1-e_{j}\right) s_{j}+e_{j} \beta\right)\right)\right]
$$

as $\lambda_{\varepsilon_{0}}=1$. 
We get

$$
\begin{aligned}
\Psi_{\frac{1}{2}(1-\alpha+\beta) i}(\alpha, \beta ; h)= & \frac{2^{d}}{i^{d} d} \sum_{\mathrm{e} \in\{ \pm 1\}^{d}} e_{1} \cdots e_{d} \int \cdots \int_{(-i \infty, i \infty)^{d}} \widetilde{h}(\underline{s} ; \mathrm{e}) \times \\
& \times \prod_{j=1}^{d}\left[\cos \left(\frac{1}{2} \pi\left(\left(1-e_{j}\right) s_{j}+e_{j} \beta\right)\right) \Gamma\left(s_{j}-1-\beta\right) \Gamma\left(1-s_{j}\right)\right] d \underline{s} .
\end{aligned}
$$

The $s_{j}$-contour separates the poles of $\Gamma\left(s_{j}-1-\beta\right)$ and $\Gamma\left(1-s_{j}\right)$ to the left and the right, respectively, and by the restriction to the domain 3.2 .45 can be drawn.

Now we let $r_{j}=\frac{1}{2}(1+\alpha+\beta) i$. Using the same arguments as above, we get

$$
\begin{aligned}
\Psi_{\frac{1}{2}(1+\alpha+\beta) i}= & \frac{2^{d}}{i^{d} d} \sum_{\mathrm{e} \in\{ \pm 1\}^{d}} e_{1} \cdots e_{d} \int_{(-i \infty, i \infty)^{d}} \ldots \int \widetilde{h}(\underline{s}+\alpha ; \mathrm{e}) \times \\
& \times \prod_{j=1}^{d}\left[\cos \left(\frac{1}{2} \pi\left(\left(1-e_{j}\right) s_{j}+e_{j} \beta\right)\right) \Gamma\left(s_{j}-1-\beta\right) \Gamma\left(1-s_{j}\right)\right] d \underline{s}
\end{aligned}
$$

where $\underline{s}+\alpha=\left(s_{1}+\alpha, \ldots, s_{d}+\alpha\right)$.

We have to compute the multiple integrals over $\underline{s}$. We consider the second one in detail, as it is a bit more complicated than the first. The first can be done following these steps.

We use the definition of $\widetilde{h}$ given in (3.2.13) and apply partial integration in each variable.

Thus, the integral equals

$$
e_{1} \cdots e_{d} \int_{(0, \infty)^{d}} \ldots \int_{d}(\mathrm{e} \underline{u}) \prod_{j=1}^{d} R\left(u_{j}, e_{j}\right) d \underline{u}
$$

where $h_{d}(\underline{u})=\left(\partial_{u_{1}} \ldots \partial_{u_{d}} h\right)(\underline{u})$,

$$
R\left(u_{j}, e_{j}\right)=\int_{-i \infty}^{i \infty} \cos \left(\frac{1}{2} \pi\left(\left(1-e_{j}\right) s_{j}+e_{j} \beta\right)\right) \Gamma\left(s_{j}-1-\beta\right) \Gamma\left(1-s_{j}\right) u_{j}^{s_{j}+\alpha} \frac{d s_{j}}{s_{j}+\alpha} .
$$

By 3.2.45 we may take for instance $\operatorname{Re} s=\frac{1}{2}$ as contour. In particular, we can assume that the pole $s=-\alpha$ is on the left of the contour.

We shift the contour in 3.2 .48 to $\operatorname{Re} s=+\infty$ or to $\operatorname{Re} s=-\infty$, depending on whether $u_{j}<1$ or $u_{j}>1$.

For $u_{j}<1$ the shift of contour to $\operatorname{Re} s=+\infty$ collects poles of $\Gamma(1-s)$, and the resulting integral vanishes. We get

$$
R\left(u_{j}, e_{j}\right)=2 \pi i e_{j} \cos \left(\frac{1}{2} \pi \beta\right) \Gamma(-\beta) \int_{0}^{u_{j}} u^{\alpha}\left(1+e_{j} u\right)^{\beta} d u
$$


If $u_{j}>1$ we collect the poles of $\Gamma(s-1-\beta)$ and the pole $s=-\alpha$. We get

$$
\begin{aligned}
R\left(u_{j}, e_{j}\right)= & -2 \pi i e_{j} \cos \left(\frac{1}{2} \pi \beta\right) \Gamma(-\beta) \int_{u_{j}}^{\infty} u^{\alpha}\left(\left|1+e_{j} u\right|^{\beta}-u^{\beta}\right) d u \\
& +2 \pi i e_{j} \cos \left(\frac{1}{2} \pi \beta\right) \Gamma(-\beta) \frac{u_{j}^{1+\alpha+\beta}}{1+\alpha+\beta} \\
& +2 \pi i \cos \left(\frac{1}{2} \pi\left(\left(e_{j}-1\right) \alpha+e_{j} \beta\right)\right) \Gamma(1+\alpha) \Gamma(-1-\alpha-\beta) .
\end{aligned}
$$

Hence, for $u_{j}>0, u_{j} \neq 1$, we have

$$
\partial_{u_{j}} R\left(u_{j}, e_{j}\right)=2 \pi i e_{j} \cos \left(\frac{1}{2} \pi \beta\right) \Gamma(-\beta) u_{j}^{\alpha}\left|1+e_{j} u_{j}\right|^{\beta} .
$$

Applying partial integration to 3.2.47) and inserting this expression for $\partial_{u_{j}} R$ we obtain

$$
\Psi_{\frac{1}{2}(1+\alpha+\beta) i}(\alpha, \beta ; h)=(2 \pi i)^{d}\left(\cos \left(\frac{1}{2} \pi \beta\right) \Gamma(-\beta)\right)^{d} \ddot{h}(\alpha, \beta)
$$

with $\ddot{h}$ as defined in the Theorem.

Analogously, we have

$$
\Psi_{\frac{1}{2}(1-\alpha+\beta) i}(\alpha, \beta ; h)=\frac{\left(2^{3} \pi\right)^{d}}{d}\left(\cos \left(\frac{1}{2} \pi \beta\right) \Gamma(-\beta)\right)^{d} \ddot{h}(0, \beta) .
$$

We insert this into 3.2.46) and use the functional equation for the Dedekind $\zeta$-function to see that 3.2 .46 changes to

$$
\begin{aligned}
& \frac{C_{e} 2^{d}}{d \pi^{d}} R_{K}\left|D_{K}\right|^{\beta} \frac{\zeta_{K}(1-\alpha) \zeta_{K}(1+\beta)}{\zeta_{K}(2-\alpha+\beta)}(N m)^{1+\beta} \sigma_{\alpha-\beta-1}(m) \ddot{h}(0, \beta) \\
+ & \frac{C_{e} 2^{d}}{d \pi^{d}} R_{K}\left|D_{K}\right|^{\beta} \frac{\zeta_{K}(1+\alpha) \zeta_{K}(1+\beta)}{\zeta_{K}(2+\alpha+\beta)}(N m)^{1+\alpha+\beta} \sigma_{-\alpha-\beta-1}(m) \ddot{h}(\alpha, \beta) .
\end{aligned}
$$

Finally we consider the residual contribution. Taking the first two summands of the decomposition of $\mathcal{B}_{m}$ given in (3.2.35) and the terms (3.2.49) coming from $\mathcal{B}_{m}^{(e)}$ we see that they coincides with $\mathcal{B}_{m}^{(r)}$.

\subsubsection{The Fourth Moment of $\zeta_{K}$}

Using the spectral decomposition of $\mathcal{B}_{m}(\alpha, \beta ; h)$ with $(\alpha, \beta)$ in a neighborhood of $(0,0)$ we can decompose $z_{2}(g, \omega ; K)$ via the relation 3.2 .7$)$. To this end, we have to verify that $h=W_{+}$satisfies the condition 3.2.17).

\section{(3.2.4) Lemma}

Let $W_{+}(y ; \gamma, \delta)$ be as defined in (3.2.8) and (3.2.3) and let

$$
\widetilde{W_{+}}(\underline{s} ; \mathrm{e} ; \gamma, \delta)=\int_{(0, \infty)^{d}} \ldots \int_{+} W_{+}(\mathrm{e} \underline{u} ; \gamma, \delta) u_{1}^{s_{1}-1} \cdots u_{d}^{s_{d}-1} d \underline{u}
$$

be the Mellin transform of $W_{+}$, with $\mathrm{e}=\left(e_{1}, \ldots, e_{d}\right), e_{j}= \pm 1$ and $\mathrm{e} \underline{u}=\left(e_{1} u_{1}, \ldots, e_{d} u_{d}\right)$. 
Then the function $\widetilde{W_{+}}(\underline{s} ; \mathrm{e} ; \gamma, \delta)$ is regular in the domain

$$
\operatorname{Re}\left(s_{j}-\gamma-\delta\right)<0, \quad j=1, \ldots, d
$$

It has an analytic continuation given by

$$
\begin{aligned}
\widetilde{W_{+}}(\underline{s} ; \mathrm{e} ; \gamma, \delta) & =R_{K} 2^{d} \prod_{j=1}^{d}\left[\Gamma\left(\gamma+\delta-s_{j}\right)\right] \sum_{\underline{\nu} \in \Lambda^{d-1}} \omega(\underline{\nu}) \int \cdots \int \widehat{\mathrm{p}}^{2}(\underline{\xi} / \bar{\nu}) \int_{\mathbb{R}^{d-1}}^{\infty} g(t) \times \\
\times & \prod_{j=1}^{d} \frac{2}{\pi}\left[\operatorname { s i n } \left(\frac{1}{2} \pi\left(\delta-i\left(t+\xi_{j}-\nu_{j}\right)\right) \cos \left(\frac{1}{2} \pi\left(\left(1-e_{j}\right)\left(\gamma-s_{j}\right)+\delta-e_{j} i\left(t+\xi_{j}-\nu_{j}\right)\right)\right) \times\right.\right. \\
\times & \left.\left.\Gamma\left(s_{j}-\gamma-i\left(t+\xi_{j}-\nu_{j}\right)\right)\right) \Gamma\left(1-\delta+i\left(t+\xi_{j}-\nu_{j}\right)\right)\right] d t d \underline{\xi}
\end{aligned}
$$

where $\xi_{d}=-\operatorname{Tr}(\underline{\xi})$ and $\nu_{d}=-\operatorname{Tr}(\underline{\nu})$ and $\bar{\nu}$ is defined in 3.1.4). The t-contour separates the poles of the factors $\Gamma\left(s_{j}-\gamma-i\left(t+\xi_{j}-\nu_{j}\right)\right)$ and those of the factors $\Gamma\left(1-\delta+i\left(t+\xi_{j}-\nu_{j}\right)\right)$ upwards and downwards, respectively; and $s_{1}, \ldots, s_{d}, \gamma, \delta$ are assumed to be such that the contour can be drawn, so $\gamma-\operatorname{Re} s_{j}$ and $\delta-1$ are bounded away from the integers.

Moreover, for all $\gamma, \delta$ bounded away from $s_{j}, j=1, \ldots, d$ we have

$$
\widetilde{W_{+}}(\underline{s} ; \mathrm{e} ; \gamma, \delta) \ll\left(1+\left|s_{1}\right|+\cdots+\left|s_{d}\right|\right)^{-C}
$$

for every fixed $C>0$.

Proof.

We recall the definitions of $W_{+}$and of $c_{\Psi}$ given in (3.2.8) and (3.2.5), respectively. Then we write out the Fourier transform of $g$ and of $\omega$ to obtain

$$
\begin{aligned}
& W_{+}(\mathrm{e} \underline{u} ; \gamma, \delta)=\frac{W\left(1+\frac{1}{\mathrm{e}} \underline{u}\right) c_{\Psi}\left(1+\frac{1}{\mathrm{e} \underline{u}}\right)}{|N \underline{u}|^{\gamma} \mid N(1+\underline{u})^{\delta}} \\
= & R_{K} \sum_{\nu \in \Lambda^{d-1}} \omega(\underline{\nu}) \int \ldots \int \widehat{\mathrm{p}}^{2}(\underline{\xi} / \bar{\nu}) \int_{\mathbb{R}^{d-1}}^{\infty} g(t) \prod_{j=1}^{d}\left|1+\frac{1}{e_{j} u_{j}}\right|^{-i \nu_{j}+i \xi_{j}+i t} u_{j}^{-\gamma}\left|1+e_{j} u_{j}\right|^{-\delta} d t d \underline{\xi} \\
= & R_{K} \sum_{\nu \in \Lambda^{d-1}} \omega(\underline{\nu}) \int \ldots \int \widehat{\mathrm{p}}^{2}(\underline{\xi} / \bar{\nu}) \int_{\mathbb{R}^{d-1}}^{\infty} g(t) \prod_{j=1}^{d} \frac{u_{j}^{-\gamma-i\left(t+\xi_{j}-\nu_{j}\right)}}{\left|1+e_{j} u_{j}\right|^{\delta-i\left(t+\xi_{j}-\nu_{j}\right)}} d t d \underline{\xi}
\end{aligned}
$$

with $u_{j}>0$ for all $j=1, \ldots, d$, and again $\xi_{d}=-\operatorname{Tr}(\underline{\xi})$ and $\nu_{d}=-\operatorname{Tr}(\underline{\nu})$.

Then by the rapid decay of $g$ in every fixed horizontal strip and the rapid decay of $\omega$ in each variable in every fixed horizontal strip we have the estimate

$$
W_{+}(\mathrm{e} \underline{u} ; \gamma, \delta) \ll(N \underline{u})^{-\gamma-\delta}
$$

for $u_{j} \uparrow \infty$ for all $j=1, \ldots, d$. Shifting the contour of the inner integral appropriately, we obtain that $W_{+}$is of rapid decay for $u_{j} \downarrow 0$ as well as for $u_{j} \rightarrow 1$ if $e_{j}=-1$, for some $j=1, \ldots, d$.

Assume for the moment that

$$
\operatorname{Re} \gamma<\operatorname{Re} s<\operatorname{Re}(\gamma+\delta)<\operatorname{Re} \gamma+1
$$


to get via Mellin transform

$$
\widetilde{W_{+}}(\underline{s} ; \mathrm{e} ; \gamma, \delta)=R_{K} 2^{d} \sum_{\nu \in \Lambda^{d-1}} \omega(\underline{\nu}) \int \ldots \int \widehat{\mathrm{p}}^{2}(\underline{\xi} / \bar{\nu}) \int_{\mathbb{R}^{d-1}}^{\infty} g(t) \prod_{j=1}^{d} b_{e_{j}}\left(s_{j}, \eta_{j} ; \gamma, \delta\right) d t d \underline{\xi}
$$

where $\eta_{j}=t+\xi_{j}-\nu_{j}$ for $j=1, \ldots, d-1$ and $\eta_{d}=t-\operatorname{Tr}(\underline{\xi})+\operatorname{Tr}(\underline{\nu})$. The factor $b_{ \pm}$is given by

$$
b_{ \pm}(s, \eta ; \gamma, \delta)=\int_{0}^{\infty} \frac{u^{s-\gamma-i \eta-1}}{|1 \pm u|^{\delta-i \eta}} d u \text {. }
$$

By the connection between the Beta- and the $\Gamma$-function, see [GR07, 3.191, 3.194.3, 8.384.1], we have, for any $\eta \in \mathbb{R}$, the identities

$$
\int_{0}^{\infty} \frac{u^{s-\gamma-i \eta-1}}{(1+u)^{\delta-i \eta}} d u=\frac{\Gamma(s-\gamma-i \eta) \Gamma(\gamma+\delta-s)}{\Gamma(\delta-i \eta)}
$$

and

$$
\int_{0}^{\infty} \frac{u^{s-\gamma-i \eta-1}}{|1-u|^{\delta-i \eta}} d u=\frac{\Gamma(s-\gamma-i \eta) \Gamma(1-\delta+i \eta)}{\Gamma(s-\gamma-\delta+1)}+\frac{\Gamma(\gamma+\delta-s) \Gamma(1-\delta+i \eta)}{\Gamma(\gamma-s+1+i \eta)} .
$$

Using the functional equation of the $\Gamma$-function in the denominator of each term, we get

$$
\begin{aligned}
b_{ \pm}(s, \eta ; \gamma, \delta)= & \frac{2}{\pi} \sin \left(\frac{1}{2} \pi(\delta-i \eta)\right) \cos \left(\frac{1}{2} \pi((1 \mp 1)(\gamma-s)+\delta \mp i \eta)\right) \times \\
& \times \Gamma(s-\gamma-i \eta) \Gamma(\gamma+\delta-s) \Gamma(1-\delta+i \eta) .
\end{aligned}
$$

Inserting this above, under the condition 3.2 .52 we obtain the second representation of $\widetilde{W_{+}}$stated in the Lemma, with integration along the real axis. Deforming the contour appropriately we may drop (3.2.52), to get the analytic continuation. Using Stirling's formula we get

$$
\widetilde{W_{+}}(\underline{s} ; \mathrm{e} ; \gamma, \delta) \ll\left(1+\left|s_{1}\right|+\cdots+\left|s_{d}\right|\right)^{-1+\operatorname{Re} \delta} .
$$

To obtain the bound 3.2 .50$)$ we move the contour appropriately regarding the poles of the $\Gamma$-functions, and again use Stirling's formula.

We recall that $\gamma=z_{1}$ and $\delta=z_{3}$ and $\alpha=z_{1}-z_{2}, \beta=z_{3}-z_{4}$. We assume initially that

$$
\begin{array}{r}
\left|\operatorname{Re}\left(z_{1}-z_{2}\right)\right|<c_{0}, \quad\left|\operatorname{Re}\left(z_{3}-z_{4}\right)\right|<c_{0} \\
\operatorname{Re} z_{1}, \operatorname{Re} z_{3}>C_{0},
\end{array}
$$

where $C_{0}$ and $c_{0}$ are sufficiently large, respectively sufficiently small positive constants.

The estimates (3.2.51), the rapid decay of $W_{+}$if at least one variable tends to 0 or -1 and 3.2 .50 ) implies that Theorem 3.2 .3 can be applied with $h=W_{+}$as 3.2 .17 is satisfied. Applying Theorem 3.2 .3 to decompose $\mathcal{B}_{m}\left(z_{1}-z_{2}, z_{3}-z_{4} ; W_{+}\left(\cdot ; z_{1}, z_{3}\right)\right)$ we get a spectral decomposition of $\mathcal{J}_{+}$. Recall the expression of $\mathcal{J}_{+}$in terms of $\mathcal{B}_{m}$ given in (3.2.7). Further we use the equality 3.1 .10 to reformulate the contribution coming from $\mathcal{B}_{m}^{(r)}$. 
We obtain

$$
\mathcal{J}_{+}\left(z_{1}, z_{2}, z_{3}, z_{4} ; g, \omega\right)=\left\{\mathcal{J}_{+}^{(r)}+\mathcal{J}_{+}^{(c)}+\mathfrak{J}_{+}^{(e)}\right\}\left(z_{1}, z_{2}, z_{3}, z_{4} ; g, \omega\right)
$$

with

$$
\begin{aligned}
& \mathcal{J}_{+}^{(r)}\left(z_{1}, z_{2}, z_{3}, z_{4} ; g, \omega\right) \\
& =\left|D_{K}\right|^{-\frac{2\left(z_{1}-z_{2}\right)-1}{4}} \frac{\zeta_{K}\left(1-z_{1}+z_{2}\right) \zeta_{K}\left(1-z_{3}+z_{4}\right)}{\zeta_{K}\left(2-z_{1}+z_{2}-z_{3}+z_{4}\right)} \zeta_{K}\left(z_{1}+z_{3}-1\right) \zeta_{K}\left(z_{2}+z_{4}\right) \ddot{W}_{+}\left(0,0 ; z_{1}, z_{3}\right) \\
& +\left|D_{K}\right|^{\frac{2\left(z_{1}-z_{2}\right)-1}{4}} \frac{\zeta_{K}\left(1+z_{1}-z_{2}\right) \zeta_{K}\left(1-z_{3}+z_{4}\right)}{\zeta_{K}\left(2+z_{1}-z_{2}-z_{3}+z_{4}\right)} \zeta_{K}\left(z_{2}+z_{3}-1\right) \zeta_{K}\left(z_{1}+z_{4}\right) \ddot{W}_{+}\left(z_{1}-z_{2}, 0 ; z_{1}, z_{3}\right) \\
& +\frac{2^{2 d} R_{K}\left|D_{K}\right|^{z_{3}-z_{4}+\frac{1}{2}}}{d \pi^{d}} \frac{\zeta_{K}\left(1-z_{1}+z_{2}\right) \zeta_{K}\left(1+z_{3}-z_{4}\right)}{\zeta_{K}\left(2-z_{1}+z_{2}+z_{3}-z_{4}\right)} \zeta_{K}\left(z_{1}+z_{4}-1\right) \zeta_{K}\left(z_{2}+z_{3}\right) \ddot{W}_{+}\left(0, z_{3}-z_{4} ; z_{1}, z_{3}\right) \\
& +\frac{2^{2 d} R_{K}\left|D_{K}\right|^{z_{3}-z_{4}+\frac{1}{2}}}{d \pi^{d}} \frac{\zeta_{K}\left(1+z_{1}-z_{2}\right) \zeta_{K}\left(1+z_{3}-z_{4}\right)}{\zeta_{K}\left(2+z_{1}-z_{2}+z_{3}-z_{4}\right)} \zeta_{K}\left(z_{2}+z_{4}-1\right) \zeta_{K}\left(z_{1}+z_{3}\right) \times \\
& \times \ddot{W}_{+}\left(z_{1}-z_{2}, z_{3}-z_{4} ; z_{1}, z_{3}\right) \text {, } \\
& \mathcal{J}_{+}^{(c)}\left(z_{1}, z_{2}, z_{3}, z_{4} ; g, \omega\right) \\
& =\frac{2(2 \pi)^{d\left(z_{3}-z_{4}\right)}}{(2 \pi)^{2 d}} \sum_{V} a_{V} \eta_{V}(1) H_{V}\left(\frac{1}{2}\left(z_{1}+z_{2}+z_{3}+z_{4}-1\right)\right) H_{V}\left(\frac{1}{2}\left(1+z_{1}-z_{2}-z_{3}+z_{4}\right)\right) \times \\
& \times H_{V}\left(\frac{1}{2}\left(1-z_{1}+z_{2}-z_{3}+z_{4}\right)\right) \Phi_{V}\left(\kappa_{V} ; z_{1}-z_{2}, z_{3}-z_{4} ; W_{+}\left(\cdot ; z_{1}, z_{3}\right)\right), \\
& \mathfrak{J}_{+}^{(e)}\left(z_{1}, z_{2}, z_{3}, z_{4} ; g, \omega\right)=\frac{2(2 \pi)^{d\left(z_{3}-z_{4}\right)}}{(2 \pi)^{2 d}} C_{e} \sum_{\underline{\nu} \in \Lambda^{d-1}} \int_{-\infty}^{\infty} \frac{Z_{K}\left(z_{1}, z_{2}, z_{3}, z_{4} ; t, \underline{\nu}\right)}{\left|\zeta_{K}(1+2 i t, 2 \underline{\nu})\right|^{2}} \times \\
& \times \Phi_{\varepsilon_{\underline{\nu}}}\left(t+\underline{\nu} ; z_{1}-z_{2}, z_{3}-z_{4} ; W_{+}\left(\cdot ; z_{1}, z_{3}\right)\right) d t
\end{aligned}
$$

with $t+\underline{\nu}=\left(t+\nu_{1}, \ldots, t+\nu_{d-1}, t-\operatorname{Tr}(\underline{\nu})\right)$ and

$$
\begin{aligned}
Z_{K}\left(z_{1}, z_{2}, z_{3}, z_{4} ; t, \nu\right) & =\zeta_{K}\left(\frac{1}{2}\left(z_{1}+z_{2}+z_{3}+z_{4}-1\right)+i t,-\underline{\nu}\right) \zeta_{K}\left(\frac{1}{2}\left(z_{1}+z_{2}+z_{3}+z_{4}-1\right)-i t, \underline{\nu}\right) \times \\
& \times \zeta_{K}\left(\frac{1}{2}\left(1+z_{1}-z_{2}-z_{3}+z_{4}\right)+i t,-\underline{\nu}\right) \zeta_{K}\left(\frac{1}{2}\left(1+z_{1}-z_{2}-z_{3}+z_{4}\right)-i t, \underline{\nu}\right) \times \\
& \times \zeta_{K}\left(\frac{1}{2}\left(1-z_{1}+z_{2}-z_{3}+z_{4}\right)+i t,-\underline{\nu}\right) \zeta_{K}\left(\frac{1}{2}\left(1-z_{1}+z_{2}-z_{3}+z_{4}\right)-i t, \underline{\nu}\right), \\
\ddot{W}_{+}\left(s_{1}, s_{2} ; z_{1}, z_{3}\right) & =\int_{\mathbb{R}^{d}} \ldots \int W_{+}\left(\underline{u} ; z_{1}, z_{3}\right)|N \underline{u}|^{s_{1}}\left(\left|1+u_{1}\right| \cdots\left|1+u_{d}\right|\right)^{s_{2}} d \underline{u},
\end{aligned}
$$

and $\Phi_{*}\left(\underline{r} ; z_{1}-z_{2}, z_{3}-z_{4} ; W_{+}\left(\cdot ; z_{1}, z_{3}\right)\right)$ as defined in 3.2 .36$)$.

To get the expressions for $\mathcal{J}_{+}^{(c)}$ and $\mathcal{J}_{+}^{(e)}$ we need the absolute convergence implied by the Lemmas 3.1.4. 3.1 .6 and 3.2 .2 ,

Now we are left with the continuation of this decomposition to a neighborhood of the central point $p_{\frac{1}{2}}=\left(\frac{1}{2}, \frac{1}{2}, \frac{1}{2}, \frac{1}{2}\right)$. The arguments are the same as for the case $d=2$ in BM01, Section 6].

First we consider the cuspidal contribution $\mathcal{J}_{+}^{(c)}$. To obtain well-definedness of $\Phi_{V}$ in the domain 3.2.53-3.2.54 we shift the $s_{j}$-contour in 3.2.36 to $\operatorname{Re} s_{j}=\frac{3}{4}$ for all $j=1, \ldots, d$. Then, by rewriting 
the domain $\operatorname{Re}\left(s_{j}-\gamma-\delta\right)$ of Lemma 3.2 .4 we see that $\Phi_{V}$ is regular in

$$
\left\{\operatorname{Re}\left(z_{1}+z_{3}\right)>\frac{3}{4} \text { and } 3.2 .53 \text { hold }\right\} \text {. }
$$

For the eigenvalues of the form $i \kappa_{j}=l_{j}-\frac{1}{2}$ a modification of $\Phi_{V}$ via (3.1.48) is needed before shifting the $s_{j}$-contours sufficiently far to the left. Then using the estimate 3.2 .50 we see that $\mathcal{J}_{+}^{(c)}$ continues to the domain 3.2.58). In particular it is regular at $p_{\frac{1}{2}}$; and the right hand side of 3.2.56) converges and represents $\mathcal{J}_{+}^{(c)}\left(p_{\frac{1}{2}} ; g, \omega\right)$.

Now we consider the Eisenstein contribution $\mathfrak{J}_{+}^{(e)}$. The terms corresponding to $\underline{\nu} \neq 0$ are analogous to the cuspidal contribution, and this part is regular in (3.2.58). Thus we are left with the term $\underline{\nu}=0$, given by

$$
\frac{2(2 \pi)^{d\left(z_{3}-z_{4}\right)}}{(2 \pi)^{2 d}} C_{e} \int_{-\infty}^{\infty} \frac{Z_{K}\left(z_{1}, z_{2}, z_{3}, z_{4} ; t\right)}{\left|\zeta_{K}(1+2 i t)\right|^{2}} \Psi_{t}\left(z_{1}-z_{2}, z_{3}-z_{4} ; W_{+}\left(\cdot ; z_{1}, z_{3}\right)\right) d t
$$

where $\Psi_{t}$ is as in (3.2.44) and $\left(z_{1}, z_{2}, z_{3}, z_{4}\right)$ satisfies 3.2.53)-(3.2.54). This continues to the domain where $\operatorname{Re}\left(z_{1}+z_{2}+z_{3}+z_{4}\right)>3$ and (3.2.53) hold. Then we follow the argument of (3.2.43). We restrict our domain to $\left\{3<\operatorname{Re}\left(z_{1}+z_{2}+z_{3}+z_{4}\right)<\frac{13}{4}\right.$ and $(3.2 .53)$ hold $\}$, and move the contour to $\operatorname{Im} t=\frac{1}{8}$. We collect poles from the factor $\zeta_{K}^{-1}(1+2 i t)$ and at $t=-\frac{1}{2}\left(z_{1}+z_{2}+z_{3}+z_{4}-3\right) i$. The resulting integral is regular in $\left\{\frac{11}{4}<\operatorname{Re}\left(z_{1}+z_{2}+z_{3}+z_{4}\right)<\frac{13}{4}\right.$ with $\left.(3.2 .53)\right\}$. After reducing the upper bound from $\frac{13}{4}$ to 3 we shift the contour back to $\mathbb{R}$, and encounter poles at $t=\frac{1}{2}\left(z_{1}+z_{2}+z_{3}+z_{4}-3\right) i$ and those from $\zeta_{K}^{-1}(1+2 i t)$.

The poles at $t= \pm \frac{1}{2}\left(z_{1}+z_{2}+z_{3}+z_{4}-3\right) i$ contribute the residual term

$$
\begin{aligned}
\frac{2(2 \pi)^{d\left(z_{3}-z_{4}\right)}}{(2 \pi)^{2 d}} C_{e} \frac{\zeta_{K}\left(2-z_{2}-z_{3}\right) \zeta_{K}\left(z_{1}+z_{4}-1\right) \zeta_{K}\left(2-z_{1}-z_{3}\right) \zeta_{K}\left(z_{2}+z_{4}-1\right)}{\zeta_{K}\left(4-z_{1}-z_{2}-z_{3}-z_{4}\right)} \times \\
\times \Psi_{\frac{1}{2}\left(z_{1}+z_{2}+z_{3}+z_{4}-3\right) i}\left(z_{1}-z_{2}, z_{3}-z_{4} ; g, \omega\right)=: \mathfrak{J}_{+}^{(e, r)}
\end{aligned}
$$

The residual terms coming from $\zeta_{K}^{-1}$ cancel.

Then the new integral is regular in the domain

$$
\left\{\operatorname{Re}\left(z_{1}+z_{2}+z_{3}+z_{4}\right)<3 \text { and } 3.2 .58 \text { hold }\right\}
$$

which contains $p_{\frac{1}{2}}$. Thus we obtain the continuation of 3.2 .59 , which is given by the same expression as 3.2.59 with the additional term 3.2.60 coming from the residues.

Let

$$
\mathfrak{J}^{(r)}=\mathcal{J}_{0}+\mathfrak{J}_{+}^{(r)}+\mathfrak{J}_{+}^{(e, r)}
$$

be the sum of $\mathcal{J}_{0}$ given in (3.2.4), the residual contribution $\mathcal{J}_{+}^{(r)}$ given in 3.2 .55 and the residual part of the continued Eisenstein contribution $\mathcal{J}_{+}^{(e, r)}$ given in 3.2 .60 . Then $\mathcal{J}^{(r)}$ has to be regular at $p_{\frac{1}{2}}$ as in a neighborhood of $p_{\frac{1}{2}}$ we have

$$
\mathcal{J}=\mathfrak{J}^{(r)}+\mathfrak{J}_{+}^{(c)}+\mathfrak{J}_{+}^{(e)},
$$

and $\mathcal{J}, \mathcal{J}_{+}^{(c)}$ and $\mathcal{J}_{+}^{(e)}$ are all regular at $p_{\frac{1}{2}}$. 
Thus, we get an explicit formula for the average values of the fourth power moment of Hecke $L$-functions of a totally real number field $K$ with class number one.

\section{(3.2.5) Theorem}

Let $K$ be a totally real number field of class number one, and let $K$ have fundamental units ${ }_{j} \epsilon$ such that every ideal has a totally positive generator. Let $\Lambda^{d-1}$ be the $(d-1)$-dimensional lattice of the Größencharacters defined by (3.1.3). Let $g(t)$ and $\omega\left(\nu_{1}, \ldots, \nu_{d-1}\right)$ be entire of rapid decay in every fixed horizontal strip for every variable.

Further let

$$
M_{K}(g, \omega)=\mathfrak{J}^{(r)}\left(p_{\frac{1}{2}}\right)+C_{K}(g, \omega)
$$

be the term coming from the residual contributions where $\mathcal{J}^{(r)}$ is defined in (3.2.61) and $C_{K}(g, \omega)$ is defined in 3.2 .2 .

Let $W_{+}$be as in $(3.2 .8)$ and $\widetilde{W_{+}}$its Mellin transform. Choose the functions $\lambda_{*}$ and $\eta_{*}$ as in Section 3.1.2. let $\varepsilon=\left(\varepsilon_{1}, \ldots, \varepsilon_{d}\right)$ be as in Lemma 3.1.6, and let $\kappa_{V}=\left(\kappa_{1}, \ldots, \kappa_{d}\right)$ and $t+\underline{\nu}=\left(t+\nu_{1}, \ldots, t+\nu_{d}\right)$ where $\nu_{d}=-\operatorname{Tr}(\underline{\nu})$. The integral transformations of $g$ and $\omega$ are given by

$$
\begin{aligned}
\Lambda_{V}(g, \omega) & =\frac{2}{(2 \pi)^{d}} \Phi_{V}\left(\kappa_{V} ; 0,0 ; W_{+}\left(\cdot ; \frac{1}{2}, \frac{1}{2}\right)\right), \\
\Xi_{\underline{\nu}}(t ; g, \omega) & =\frac{2}{(2 \pi)^{d}} \Phi_{\varepsilon_{\underline{\nu}}}\left(t+\underline{\nu} ; 0,0 ; W_{+}\left(\cdot ; \frac{1}{2}, \frac{1}{2}\right)\right)
\end{aligned}
$$

where

$$
\begin{aligned}
\Phi_{*}(\underline{r} ; 0,0 ; & \left.W_{+}\left(\cdot ; \frac{1}{2}, \frac{1}{2}\right)\right)=\frac{\eta_{*}(1)}{(2 i)^{d} d} \sum_{\mathrm{e} \in\{ \pm 1\}^{d}} \int_{\left(\frac{3}{4}\right) \ldots\left(\frac{3}{4}\right)} \ldots \int \widetilde{W_{+}}\left(\underline{s} ; \mathrm{e} ; \frac{1}{2}, \frac{1}{2}\right) \times \\
& \times \prod_{j=1}^{d}\left[\Gamma\left(1-s_{j}\right)^{2} \Gamma\left(s_{j}-\frac{1}{2}-i r_{j}\right) \Gamma\left(s_{j}-\frac{1}{2}+i r_{j}\right) \Delta_{*}\left(\varepsilon_{j}, e_{j} ; s_{j}, r_{j}\right)\right] d \underline{s},
\end{aligned}
$$

with

$$
\begin{aligned}
\Delta_{*}\left(\varepsilon_{j}, e_{j} ; s, r\right)= & {\left[\left(1+e_{j}\right)-\left(1-e_{j}\right) \cos (\pi s)\right] 2 \sin (\pi s) } \\
& +\lambda_{*}\left(\varepsilon_{j}\right)\left[\left(1-e_{j}\right)-\left(1+e_{j}\right) \cos (\pi s)\right] 2 \cosh (\pi r) .
\end{aligned}
$$

Then we have

$$
\begin{aligned}
& \sum_{\underline{\nu} \in \Lambda^{d-1}} \omega(\underline{\nu}) \int_{-\infty}^{\infty}\left|\zeta_{K}\left(\frac{1}{2}+i t, \underline{\nu}\right)\right|^{4} g(t) d t=M_{K}(g, \omega) \\
& +\sum_{V} a_{V} \eta_{V}(1) H_{V}\left(\frac{1}{2}\right)^{3} \Lambda_{V}(g, \omega)+2^{d} \sqrt{\left|D_{K}\right|} \sum_{\underline{\nu} \in \Lambda^{d-1}} \int_{-\infty}^{\infty} \frac{\left|\zeta_{K}\left(\frac{1}{2}+i t, \underline{\nu}\right)\right|^{6}}{\left|\zeta_{K}(1+2 i t, 2 \underline{\nu})\right|^{2}} \Xi_{\underline{\nu}}(t ; g, \omega) d t
\end{aligned}
$$

where $t_{V}, \eta_{V}, a_{V}, H_{V}$ are defined in Section 3.1.2. The sum $V$ runs over an orthonormal system of Hecke invariant cuspidal irreducible subspaces of $L^{2}\left(\Gamma \backslash \mathrm{PSL}_{2}(\mathbb{R})^{d}\right)$ with $\Gamma$ the Hilbert modular group over $K$. 


\subsection{A Bound for The Fourth Moment}

In this section we prove an asymptotic formula for the average value for the fourth moment of Hecke $L$-functions for a totally real number field $K$ of degree $d$ with class number one, given by

$$
\sum_{\underline{\nu} \in \Lambda^{d-1}} \omega(\underline{\nu}) \int_{-\infty}^{\infty}\left|\zeta_{K}\left(\frac{1}{2}+i t, \underline{\nu}\right)\right|^{4} g(t) d t
$$

using the decomposition we proved in the previous section (Theorem 3.2.5).

We choose the functions $g(t)$ and $\omega\left(\nu_{1}, \ldots, \nu_{d-1}\right)$ as before to be entire functions, of rapid decay in every horizontal strip. More precisely, we fix two entire functions $G$ and $\Omega$ that are of rapid decay and satisfy the estimates

$$
\begin{aligned}
G^{(j)}(x) & \ll_{A, j}(1+|x|)^{-A}, \\
\frac{\partial^{j_{1}}}{\partial y_{1}^{j_{1}}} \cdots \frac{\partial^{j_{d-1}}}{\partial y_{d-1}^{j_{d-1}}} \Omega(\underline{y}) & \ll_{A, \underline{j}}\left(1+\left|y_{1}\right|+\cdots+\left|y_{d-1}\right|\right)^{-A}
\end{aligned}
$$

with $A>2$ for all $j, j_{1}, \ldots, j_{d-1} \in \mathbb{N}_{0}$.

Then let $g(t)=G\left(\frac{t}{T}\right)$ and $\omega(\underline{\nu})=\Omega\left(\frac{\nu_{1}}{T}, \ldots, \frac{\nu_{d-1}}{T}\right)=\Omega(\underline{\nu} / T)$.

According to Theorem 3.2 .5 we have to estimate the expression

$$
\begin{aligned}
& M_{K}(g, \omega) \\
+ & \sum_{V} a_{V} \eta_{V}(1) H_{V}\left(\frac{1}{2}\right)^{3} \Lambda_{V}(g, \omega)+2^{d} \sqrt{\left|D_{K}\right|} \sum_{\underline{\nu} \in \Lambda^{d-1}} \int_{-\infty}^{\infty} \frac{\left|\zeta_{K}\left(\frac{1}{2}+i t, \underline{\nu}\right)\right|^{6}}{\left|\zeta_{K}(1+2 i t, 2 \underline{\nu})\right|^{2}} \Xi_{\underline{\nu}}(t ; g, \omega) d t .
\end{aligned}
$$

The functions $\Lambda_{V}$ and $\Xi_{\underline{\nu}}$ are integral transforms of the functions $g$ and $\omega$, see Theorem 3.2.5. The term $M_{K}(g, \omega)$ contributes the main term.

\subsubsection{Error Term}

First, we consider the second and the third term of 3.3.2 which enter into the error term.

There exist bounds for all $H_{V}$ and the sum over $a_{V}, \eta_{V}$ combined with some sufficiently nice function, see Lemma 3.1.4 and 3.1.6. Also the $\zeta$-functions coming up in the third term can be bounded. Thus we are left with finding bounds for the integral transforms $\Lambda_{V}$ and $\Xi_{\underline{\nu}}$, that are given by

$$
\begin{aligned}
\Phi_{*}(\underline{r} ; 0,0 ; & \left.W_{+}\left(\cdot ; \frac{1}{2}, \frac{1}{2}\right)\right)=\frac{\eta_{*}(1)}{(2 i)^{d} d} \sum_{\mathrm{e} \in\{ \pm 1\}^{d}} \int_{\left(\frac{3}{4}\right) \ldots\left(\frac{3}{4}\right)} \ldots \int \widetilde{W_{+}}\left(\underline{s} ; \mathrm{e} ; \frac{1}{2}, \frac{1}{2}\right) \times \\
\times & \prod_{j=1}^{d}\left[\Gamma\left(1-s_{j}\right)^{2} \Gamma\left(s_{j}-\frac{1}{2}-i r_{j}\right) \Gamma\left(s_{j}-\frac{1}{2}+i r_{j}\right) \Delta_{*}\left(\varepsilon_{j}, e_{j} ; s_{j}, r_{j}\right)\right] d \underline{s},
\end{aligned}
$$

with $\underline{r}=\kappa_{V}$ or $t+\underline{\nu}$ and

$\Delta_{*}\left(\varepsilon_{j}, e_{j} ; s, r\right)=\left[\left(1+e_{j}\right)-\left(1-e_{j}\right) \cos (\pi s)\right] 2 \sin (\pi s)+\lambda_{*}\left(\varepsilon_{j}\right)\left[\left(1-e_{j}\right)-\left(1+e_{j}\right) \cos (\pi s)\right] 2 \cosh (\pi r)$.

The function $W_{+}$is defined in 3.2 .8 and its Mellin transform $\widetilde{W_{+}}$is given in Lemma 3.2.4. We need 
the following estimate on $\widetilde{W_{+}}$to bound $\Phi_{*}$.

\section{(3.3.1) Lemma}

Let $\widetilde{W_{+}}$be defined as in Lemma 3.2.4 with $W_{+}$defined in 3.2 .8 . The function $\widetilde{W_{+}}\left(\underline{s} ; \mathrm{e} ; \frac{1}{2}, \frac{1}{2}\right)$ is holomorphic in $\operatorname{Re} s_{j}<1$ for $j=1, \ldots, d$. In this region, for every $\alpha \in \mathbb{N}$ we have the bound

$$
\widetilde{W_{+}}\left(\underline{s} ; \mathrm{e} ; \frac{1}{2}, \frac{1}{2}\right) \ll T^{\operatorname{Re} s_{1}+\cdots+\operatorname{Re} s_{d}}\left(1+\left|s_{1}\right|+\cdots+\left|s_{d}\right|\right)^{-\alpha} .
$$

Proof.

Fix $s_{j}$ for $j=1, \ldots, d$ with $\operatorname{Re} s_{j}<1$. For $w_{j} \in \mathbb{R}$ we define the function

$$
\mathcal{W}\left(1+\frac{1}{\underline{w}}\right):=W_{+}\left(\underline{w} ; \frac{1}{2}, \frac{1}{2}\right)=\frac{W\left(1+\frac{1}{\underline{w}}\right) c_{\Psi}\left(1+\frac{1}{\underline{w}}\right)}{\left(\left|1+w_{1}\right| \cdots\left|1+w_{d}\right| \cdot N \underline{w}\right)^{\frac{1}{2}}}
$$

where $1+\frac{1}{\underline{w}}=\left(1+\frac{1}{w_{1}}, \ldots, 1+\frac{1}{w_{d}}\right)$.

We recall that $W(\underline{x})$ vanishes if $x_{j} \leq 0$ for at least one $j=1, \ldots, d$. Recall that the function $W$ is a combination of the Fourier transformations of $g$ and $\omega$, see (3.2.3). The assumptions (3.3.1) and the properties of the Fourier transformation combined with the decay behavior of $c_{\Psi}$ imply that $\mathcal{W}(\underline{x})$ is rapidly decaying if $\left|x_{j}-1\right|>\frac{1}{T}$ for at least one $j$. Thus we obtain that $\mathcal{W}\left(1+\frac{1}{\underline{w}}\right)$ is of rapid decay if $\left|w_{j}\right|<T$ for at least one $j=1, \ldots, d$.

Moreover we obtain the bounds

$$
\begin{aligned}
\mathcal{W}(\underline{x})=\frac{W(\underline{x}) c_{\Psi}(x)|N(\underline{x}-1)|}{(N \underline{x})^{\frac{1}{2}}} & \ll \frac{T^{d} \frac{1}{T^{d}}}{1} \ll 1, \\
\frac{\partial^{A_{1}}}{\partial x_{1}^{A_{1}}} \cdots \frac{\partial^{A_{d}}}{\partial x_{d}^{A_{d}}} \mathcal{W}(\underline{x}) & \ll B, A_{j} T^{-A_{1}-\cdots-A_{d}} \prod_{j=1}^{d}\left(1+\frac{T}{\left|x_{j}\right|}\right)^{-B} .
\end{aligned}
$$

The first bound follows by the decay properties of $g$ and $\omega$, and the second is implied by the first and the properties of the Fourier transform.

Next we apply integration by parts in the $w_{j}$-variable $A_{j}$-times. More precisely, we choose

$$
A_{j}= \begin{cases}0, & \text { if } \operatorname{Im} s_{j} \text { near } 0 \\ \text { large, } & \text { otherwise. }\end{cases}
$$

We get

$$
\begin{aligned}
\widetilde{W_{+}}\left(\underline{s} ; \mathrm{e} ; \frac{1}{2}, \frac{1}{2}\right) & =\int_{(0, \infty)^{d}} \ldots \int_{\frac{\partial^{A_{1}}}{\partial x_{1}^{A_{1}}}} \cdots \frac{\partial^{A_{d}}}{\partial x_{d}^{A_{d}}}\left(\mathcal{W}\left(1+\frac{1}{\underline{w}}\right)\right) \prod_{j=1}^{d} \frac{w_{j}^{s_{j}-1+A j}}{s_{j} \cdots\left(s_{j}-1+A_{j}\right)} d \underline{w} \\
& \ll T^{\operatorname{Re} s_{1}+\cdots+\operatorname{Re} s_{d}}\left(1+\left|s_{1}\right|\right)^{-A_{1}} \cdots\left(1+\left|s_{d}\right|\right)^{-A_{d}} .
\end{aligned}
$$

Now if $\operatorname{Im} s_{j}$ is near zero the expression $\left(1+\left|s_{j}\right|\right)^{-\alpha}$ is of size one. Thus choosing the large $A_{j}$ to be $\alpha$ ends the proof. 
We also need a bound for the $\Gamma$-functions. Stirling's formula gives

$$
\begin{aligned}
& \prod_{j=1}^{d}\left[\Gamma\left(1-s_{j}\right)^{2} \Gamma\left(s_{j}-\frac{1}{2}-i r_{j}\right) \Gamma\left(s_{j}-\frac{1}{2}+i r_{j}\right) \Delta_{*}\left(\varepsilon_{j}, e_{j} ; s_{j}, r_{j}\right)\right] \\
\ll & \left(1+\left|s_{1}\right|+\cdots+\left|s_{d}\right|\right)^{\mathcal{O}(1)} \prod_{j=1}^{d}\left(1+\left|r_{j}\right|\right)^{2 \sigma_{j}-2}
\end{aligned}
$$

where $\sigma_{j}=\operatorname{Re} s_{j}$.

Now we are ready to estimate the functions $\Phi_{*}\left(\underline{r} ; 0,0 ; W_{+}\left(\cdot ; \frac{1}{2}, \frac{1}{2}\right)\right)$, with the possible values $\kappa_{V}$ and $t+\underline{\nu}$ for $\underline{r}$.

\section{(3.3.2) Lemma}

Let $\underline{r}=\left(r_{1}, \ldots, r_{d}\right)$ where each $r_{j}$ is either real or $0<i r_{j}<\frac{7}{64}$ or $i r_{j}=l_{j}-\frac{1}{2}$ with $l_{j} \in \mathbb{N}$. Then the function $\Phi_{*}\left(\underline{r} ; 0,0 ; W_{+}\left(\cdot ; \frac{1}{2}, \frac{1}{2}\right)\right)$, given in 3.3 .3$)$, is bounded by

$$
\Phi_{*}\left(\underline{r} ; 0,0 ; W_{+}\left(\cdot ; \frac{1}{2}, \frac{1}{2}\right)\right) \ll T^{d \theta+\frac{d}{2}}\left(1+\left|r_{1}\right|+\cdots+\left|r_{d}\right|\right)^{-20-d},
$$

where $\theta=\frac{7}{64}$ is an upper bound for the exceptional eigenvalues.

If all $r_{j}$ are real, we can choose $\theta=0$.

Proof.

We move the contour of the $s_{j}$-integrals left to the vertical line $\operatorname{Re} s_{j}=-(20+d)$, for all $j$. We collect residues at $s_{j}=-\mu_{j}+\frac{1}{2} \mp i r_{j}$, with $\mu_{j} \in \mathbb{N}_{0}$ and $\operatorname{Re}\left(-\mu_{j}+\frac{1}{2} \mp i r_{j}\right)>-(20+d)$. If $i r_{j}=l_{j}-\frac{1}{2}$ with $l_{j}>22+d$ we do not pass through poles. Now we have at most $2^{d}$ terms namely

$$
\prod_{j=1}^{d}\left(\sum_{\mu_{j}} \text { residue }+\int_{(-20-d)} d s_{j}\right) .
$$

We estimate the term coming from the residues and the shifted integral using Stirling's formula for the factor of $\Gamma$-functions. For the integral term, this estimate is given in (3.3.4). To estimate the function $\widetilde{W_{+}}$we use Lemma 3.3.1. Combining the estimates of the terms we obtain

$$
\begin{aligned}
& \Phi_{*}\left(\underline{r} ; 0,0 ; W_{+}\left(\cdot ; \frac{1}{2}, \frac{1}{2}\right)\right) \\
& \ll \prod_{j=1}^{d} \begin{cases}T^{\frac{1}{2}}\left(1+\left|r_{j}\right|\right)^{-20-d}+T^{-20-d}\left(1+\left|r_{j}\right|\right)^{-38-2 d}, & r_{j} \in \mathbb{R}, \\
T^{-l_{j}+1}+T^{-19-d}\left(1+\left|r_{j}\right|\right)^{-38-2 d}+\left(1+\left|r_{j}\right|\right)^{-1}, & i r_{j}=l_{j}-\frac{1}{2}, \text { and } l_{j} \leq 22+d, \\
T^{-20-d}\left(1+\left|r_{j}\right|\right)^{-38-2 d}, & i r_{j}=l_{j}-\frac{1}{2}, \text { and } l_{j}>22+d, \\
T^{\left|r_{j}\right|+\frac{1}{2}}+T^{-19-d}\left(1+\left|r_{j}\right|\right)^{-38-2 d}, & 0<i r_{j}<\frac{7}{64} .\end{cases}
\end{aligned}
$$

Comparing the factors, we see that this can be estimated by

$$
\prod_{j=1}^{d} \begin{cases}T^{\frac{1}{2}}\left(1+\left|r_{j}\right|\right)^{-20-d}, & r_{j} \in \mathbb{R}, \\ 1, & i r_{j}=l_{j}-\frac{1}{2} \text { and } l_{j} \leq 22+d, \\ T^{-20-d}\left(1+\left|r_{j}\right|\right)^{-38-2 d}, & i r_{j}=l_{j}-\frac{1}{2} \text { and } l_{j}>22+d, \\ T^{\theta+\frac{1}{2}}, & 0<i r_{j}<\frac{7}{64} .\end{cases}
$$


Combining these bounds, by noting that $1 \ll T^{\frac{1}{2}}\left(1+\left|r_{j}\right|\right)^{-20-d}$ if $i r_{j}=l_{j}-\frac{1}{2}$ and $l_{j} \leq 22$, we get

$$
\Phi_{*}\left(\underline{r} ; 0,0 ; W_{+}\left(\cdot ; \frac{1}{2}, \frac{1}{2}\right)\right) \ll T^{d \theta+\frac{d}{2}}\left(1+\left|r_{1}\right|+\cdots+\left|r_{d}\right|\right)^{-20-d}
$$

where $\theta=\frac{7}{64}$.

In particular if all $r_{j}$ are real, we get

$$
\Phi_{*}\left(\underline{r} ; 0,0 ; W_{+}\left(\cdot ; \frac{1}{2}, \frac{1}{2}\right)\right) \ll T^{\frac{d}{2}}\left(1+\left|r_{1}\right|+\cdots+\left|r_{d}\right|\right)^{-20-d} .
$$

Now we are ready to estimate the second and the third term of 3.3 .2 .

First we consider the cuspidal contribution

$$
\sum_{V} a_{V} \eta_{V}(1) H_{V}\left(\frac{1}{2}\right)^{3} \Lambda_{V}(g, \omega)
$$

We use Lemma 3.1 .6 to estimate the Hecke function $H_{V}$, and Lemma 3.2 .2 for the integral transform $\Lambda_{V}$. Inserting these estimates we get

$$
\sum_{V} a_{V} \eta_{V}(1) H_{V}\left(\frac{1}{2}\right)^{3} \Lambda_{V}(g, \omega) \ll T^{\frac{d}{2}+d \theta} \sum_{V} a_{V}\left|\eta_{V}\right|\left(1+\left|\kappa_{1}\right|+\cdots+\left|\kappa_{d}\right|\right)^{-10-d} .
$$

Then Lemma 3.1 .4 shows that the resulting sum is of constant size, and we get in total

$$
\sum_{V} a_{V} \eta_{V}(1) H_{V}\left(\frac{1}{2}\right)^{3} \Lambda_{V}(g, \omega) \ll T^{\frac{d}{2}+d \theta}
$$

For the Eisenstein contribution, i.e. the third term of (3.3.2), we need some further results to get rid of the $\zeta$-functions that we considered in Section 3.1.

The factor $\left(\zeta_{K}(1+2 i t, 2 \underline{\nu})\right)^{-1}$ is discussed in Lemma 3.1.1 and an estimate for the $\zeta$-function on the central line is stated in (3.1.6). Now let $\underline{r}=t+\underline{\nu}$ with $\underline{\nu} \in \Lambda^{d-1}$. Denote by $\nu_{d}=-\operatorname{Tr}(\underline{\nu})$. Then every $r_{j}$ is real and from Lemma 3.2 .2 we get the estimate

$$
\Xi_{\underline{\nu}}(t ; g, \omega) \ll T^{\frac{d}{2}}\left(1+\left|t+\nu_{1}\right|+\cdots+\left|t+\nu_{d}\right|\right)^{-20-d} .
$$

Inserting these estimates we obtain

$$
\begin{aligned}
& C_{e} \sum_{\underline{\nu} \in \Lambda^{d-1}} \int_{-\infty}^{\infty} \frac{\left|\zeta_{K}\left(\frac{1}{2}+i t, \underline{\nu}\right)\right|^{6}}{\left|\zeta_{K}(1+2 i t, 2 \underline{\nu})\right|^{2}} \Xi_{\underline{\nu}}(t ; g, \omega) d t \\
\ll & \sum_{\underline{\nu} \in \Lambda^{d-1}-\infty} \int_{-\infty}^{\infty}\left(1+\left|t+\nu_{1}\right|+\cdots+\left|t+\nu_{d}\right|\right)^{1+2 \varepsilon-20-d^{2}} T^{\frac{d}{2}} d t \ll T^{\frac{d}{2}} .
\end{aligned}
$$

Inserting the estimates for the cuspidal and the Eisenstein contribution into 3.3 .2 we get, with $\theta=\frac{7}{64}$, the intermediate result

$$
\sum_{\underline{\nu} \in \Lambda^{d-1}} \omega(\underline{\nu}) \int_{-\infty}^{\infty}\left|\zeta_{K}\left(\frac{1}{2}+i t, \underline{\nu}\right)\right|^{4} g(t) d t=M_{K}(g, \omega)+\mathcal{O}\left(T^{\frac{d}{2}+d \theta}\right) .
$$


It remains to estimate the summand $M_{K}(g, \omega)$, this will be done in the following subsection.

\subsubsection{Main Term}

The term $M_{K}(g, \omega)$ will give the main contribution. It is defined in Theorem 3.2 .5 as the sum

$$
M_{K}(g, \omega)=\mathfrak{J}^{(r)}\left(p_{\frac{1}{2}} ; g, \omega\right)+C_{K}(g, \omega)
$$

The term $\mathcal{J}^{(r)}$ and the constant $C_{K}(g, \omega)$ are defined in 3.2 .61 and 3.2 .2 respectively. The former consists of three term that are given in (3.2.4), (3.2.55) and (3.2.60). As mentioned above, $\mathfrak{J}^{(r)}$ is regular at $p_{\frac{1}{2}}$. We prove

\section{(3.3.3) Lemma}

Let $\mathcal{J}^{(r)}\left(p_{\frac{1}{2}} ; g, \omega\right)$ be as defined in 3.2.61), define the variables $\eta_{j}$ by $\eta_{j}=t+\xi_{j}-\nu_{j}$ for $j=1, \ldots, d-1$ and $\eta_{d}=t-\operatorname{Tr}(\underline{\xi})+\operatorname{Tr}(\underline{\nu})$. Then, the following equality holds:

$$
\begin{aligned}
\mathcal{J}^{(r)}\left(p_{\frac{1}{2}} ; g, \omega\right) & =\sum_{\underline{\nu} \in \Lambda^{d-1}} \Omega(\underline{\nu} / T) \int \ldots \int \widehat{\mathrm{p}}^{2}(\underline{\xi} / \bar{\nu}) \int_{\mathbb{R}^{d-1}}^{\infty} G\left(\frac{t}{T}\right) \times \\
& \times \sum_{\substack{a_{j}, b_{j}, k_{j}, l_{j} \geq 0 \\
j=1, \ldots, d \\
\operatorname{Tr}(a k+b l) \leq 3+d}} d(\underline{a}, \underline{k} ; \underline{b}, \underline{l}) \prod_{j=1}^{d}\left(\frac{\Gamma^{\left(a_{j}\right)}}{\Gamma}\right)^{k_{j}}\left(\frac{\Gamma^{\left(b_{j}\right)}}{\Gamma}\right)^{l_{j}}\left(\frac{1}{2}-i \eta_{j}\right) d t d \xi \\
& +\mathcal{O}\left(T^{\frac{d}{2}+\varepsilon}\right)
\end{aligned}
$$

where $\operatorname{Tr}(a k+b l)$ is the sum $\sum_{j=1}^{d}\left(a_{j} k_{j}+b_{j} l_{j}\right)$, and $d(\underline{a}, \underline{k} ; \underline{b}, \underline{l})$ are real constants that can be made explicit.

Proof.

The proof follows the corresponding steps of the case $K=\mathbb{Q}$ in [M97, pages $174-178]$.

The function $\mathcal{J}^{(r)}\left(p_{\frac{1}{2}} ; g, \omega\right)$ is regular, but its summands $\mathcal{J}_{0}, \mathcal{J}_{+}^{(r)}$ and $\mathcal{J}_{+}^{(e, r)}$ each have a singularity at $p_{\frac{1}{2}}$. Thus the singular parts cancel under addition.

More precisely, we put $\left(z_{1}, z_{2}, z_{3}, z_{4}\right)=p_{\frac{1}{2}}+\left(\delta_{1}, \delta_{2}, \delta_{3}, \delta_{4}\right)$, where $\left(\delta_{1}, \delta_{2}, \delta_{3}, \delta_{4}\right)=\left(a_{1}, a_{2}, a_{3}, a_{4}\right) \delta$ with $a_{j} \in \mathbb{R}$ and $\delta \in \mathbb{C}$ small, and we expand the terms into a Laurent series in $\delta$. Then $\mathcal{J}^{(r)}\left(p_{\frac{1}{2}} ; g, \omega\right)$ is equal to the sum of the constant terms, which does not depend on the choice of the $a_{j}$.

We compute the constant terms of $\mathcal{J}_{0}, \mathcal{J}_{+}^{(r)}$ and $\mathcal{J}_{+}^{(e, r)}$ separately.

\section{The term $J_{0}$ :}

The function $\mathcal{J}_{0}\left(z_{1}, z_{2}, z_{3}, z_{4} ; g, \omega\right)$ is given in 3.2 .4 , it is

$$
\mathcal{J}_{0}\left(z_{1}, z_{2}, z_{3}, z_{4} ; g, \omega\right)=\frac{1}{2^{d-1}} c_{\Psi} W(\underline{1}) \frac{\zeta_{K}\left(z_{1}+z_{3}\right) \zeta_{K}\left(z_{1}+z_{4}\right) \zeta_{K}\left(z_{2}+z_{3}\right) \zeta_{K}\left(z_{2}+z_{4}\right)}{\zeta_{K}\left(z_{1}+z_{2}+z_{3}+z_{4}\right)} .
$$

We use the definition of $W(\underline{1})$, write out the Fourier transform $\widehat{g}$ and apply the Poisson summation formula to $\sum_{h} \widehat{w}(h)$. Further, we recall the definition of $c_{\Psi}$. Then, the constant term of $\mathcal{J}_{0}\left(p_{\frac{1}{2}} ; g, \omega\right)$ is of the form stated in the lemma, with $a_{j}=b_{j}=0$. 


\section{The term $\mathcal{J}_{+}^{(r)}$ :}

The function $\mathcal{J}_{+}^{(r)}\left(p_{\frac{1}{2}} ; g, \omega\right)$ is given in 3.2 .55 , it is a sum of four terms, each of which is a product of $\zeta_{K}$-functions and the function $\ddot{W}_{+}\left(s_{1}, s_{2} ; z_{1}, z_{3}\right)$. One easily sees by the definition that $\ddot{W}_{+}$has a pole of order $d$ at $p_{\frac{1}{2}}$. Hence, it is essential to understand $\ddot{W}_{+}\left(s_{1}, s_{2} ; z_{1}, z_{2}\right)$ in order to compute $\mathcal{J}_{+}^{(r)}\left(p_{\frac{1}{2}} ; g, \omega\right)$. We imitate the steps of the reformulation of the function $\widetilde{W_{+}}$in the proof of Lemma 3.2.4. This leads to

$$
\begin{aligned}
& \ddot{W}_{+}\left(s_{1}, s_{2} ; z_{1}, z_{3}\right) \\
& =R_{K} \Gamma\left(-s_{1}-s_{2}+z_{1}+z_{3}-1\right)^{d} \sum_{\underline{\nu} \in \Lambda^{d-1}} \omega(\underline{\nu}) \int \cdots \int \widehat{\mathrm{p}}^{2}(\underline{\xi} / \bar{\nu}) \int_{-\infty}^{\infty} g(t) \times \\
& \quad \times \prod_{j=1}^{d} \frac{\Gamma\left(s_{1}-z_{1}-i \eta_{j}+1\right)}{\Gamma\left(-s_{2}+z_{3}-i \eta_{j}\right)}\left[1+\frac{\sin \left(\pi\left(s_{1}+s_{2}-z_{1}-z_{3}+2\right)\right)+\sin \left(\pi\left(-s_{1}+z_{1}+i \eta_{j}\right)\right)}{\sin \left(\pi\left(-s_{2}+z_{3}-i \eta_{j}\right)\right.}\right] d t d \xi .
\end{aligned}
$$

As $g$ and $\omega$ are rapidly decaying and by the usual estimates, we see that integral and sum converge absolutely.

In (3.2.55) the pair $\left(s_{1}, s_{2}\right)$ takes the values $(0,0),\left(z_{1}-z_{2}, 0\right),\left(0, z_{3}-z_{4}\right)$ and $\left(z_{1}-z_{2}, z_{3}-z_{4}\right)$. Now, we insert $z_{j}=\frac{1}{2}+\delta_{j}$ and note that $\mathcal{J}_{+}^{(r)}$ has a singularity of order $3+d$. Indeed, each of the summands can be expressed as a product over three Dedekind $\zeta_{K}$-functions, that each have pole of order one at $p_{\frac{1}{2}}$ and the functions $\ddot{W}_{+}$with a pole of order $d$. Hence, its constant term is a linear combination of the first $4+d$ coefficients of the power series in $\delta$ that is given in the second line of the right hand side of (3.3.7). Recalling the definitions of $g$ and $\omega$ we get

$$
\begin{aligned}
& \sum_{\underline{\nu} \in \Lambda^{d-1}} \Omega(\underline{\nu} / T) \int \ldots \int \widehat{\mathrm{p}}^{2}(\underline{\xi} / \bar{\nu}) \int_{\mathbb{R}^{d-1}}^{\infty} G\left(\frac{t}{T}\right) \times \\
& \times \sum_{\substack{a_{j}, b_{j}, k_{j}, l_{j} \geq 0 \\
j=1, \ldots, d \\
\operatorname{Tr}(a k+b l) \leq 3+d}} d(\underline{a}, \underline{k} ; \underline{b}, \underline{l}) \prod_{j=1}^{d}\left(\frac{\Gamma^{\left(a_{j}\right)}}{\Gamma}\right)^{k_{j}}\left(\frac{\Gamma^{\left(b_{j}\right)}}{\Gamma}\right)^{l_{j}}\left(\frac{1}{2}-i \eta_{j}\right) d t d \xi
\end{aligned}
$$

for some constants $d(\underline{a}, \underline{k} ; \underline{b}, \underline{l}) \in \mathbb{R}$ that are not necessarily the same as in the Lemma. The terms of higher order in $\delta$ contribute to the error term.

The term $\mathcal{J}_{+}^{(e, r)}$ :

The function $\mathcal{J}_{+}^{(e, r)}\left(z_{1}, z_{2}, z_{3}, z_{4} ; g, \omega\right)$ is given by

$$
\begin{aligned}
& \frac{2(2 \pi)^{d\left(z_{3}-z_{4}\right)}}{(2 \pi)^{2 d}} C_{e} \frac{\zeta_{K}\left(2-z_{2}-z_{3}\right) \zeta_{K}\left(z_{1}+z_{4}-1\right) \zeta_{K}\left(2-z_{1}-z_{3}\right) \zeta_{K}\left(z_{2}+z_{4}-1\right)}{\zeta_{K}\left(4-z_{1}-z_{2}-z_{3}-z_{4}\right)} \times \\
& \times \Phi_{\varepsilon_{0}}\left(\frac{1}{2}\left(z_{1}+z_{2}+z_{3}+z_{4}-3\right) i, \frac{1}{2}\left(z_{1}+z_{2}+z_{3}+z_{4}-3\right) i ; z_{1}-z_{2}, z_{3}-z_{4} ; g, \omega\right),
\end{aligned}
$$

see 3.2 .60 .

The function $\Phi_{\varepsilon_{0}}$ is regular at $\delta=0$, which follows by a slight shift of the $s_{j}$ contour to the left or to the right, depending on the value of $\delta$. In addition, it can be bounded by $\ll T^{\frac{d}{2}+|\delta|}$ as in the previous Section 3.3.1. By Cauchy's integral formula, this bound holds true also for the derivatives of $\Phi_{\varepsilon_{0}}$. Then, using the Laurent series of $\zeta_{K}(1+\delta)$ we see that this term contributes to the error term. 
Now we return to $M_{K}(g, \omega)$. As we said above, $M_{K}(g, \omega)$ consists of two summands, namely $\mathfrak{J}^{(r)}$ and $C_{K}(g, \omega)$.

The latter one is a constant and given by

$$
\begin{aligned}
C_{K}(g, \omega) & =\omega(\underline{0})\left(a_{0} g\left(\frac{1}{2} i\right)+b_{0} g\left(-\frac{1}{2} i\right)+a_{1} g^{\prime}\left(\frac{1}{2} i\right)+b_{1} g^{\prime}\left(-\frac{1}{2} i\right)\right) \\
& =\mathcal{O}(1) .
\end{aligned}
$$

To get an asymptotic formula for $\mathfrak{J}^{(r)}\left(p_{\frac{1}{2}} ; g, \omega\right)$ we use the representation we proved in Lemma 3.3.3. We start by computing the single terms appearing in the integral. To this end, we rewrite the sum over

$$
\left(\frac{\Gamma^{\left(a_{j}\right)}}{\Gamma}\right)^{k_{j}}\left(\frac{\Gamma^{\left(b_{j}\right)}}{\Gamma}\right)^{l_{j}}\left(\frac{1}{2}-i \eta_{j}\right)
$$

into a sum over Polygamma functions $\psi^{(n)}$ that are defined by

$$
\begin{aligned}
& \psi^{(0)}(z)=\frac{d}{d z} \log (\Gamma(z)), \\
& \psi^{(n)}(z)=\frac{d}{d z} \psi^{(n-1)}(z), \quad \text { for } n \geq 1,
\end{aligned}
$$

for example, see [GR07, 8.360]. Thus, the sum of (3.3.6 equals

$$
\begin{aligned}
& \sum_{\substack{a_{j}, b_{j}, k_{j}, l_{j} \geq 0 \\
j=1, \ldots, d \\
\operatorname{Tr}(a k+b l) \leq 3+d}} d(\underline{a}, \underline{k} ; \underline{b}, \underline{l}) \prod_{j=1}^{d}\left(\frac{\Gamma^{\left(a_{j}\right)}}{\Gamma}\right)^{k_{j}}\left(\frac{\Gamma^{\left(b_{j}\right)}}{\Gamma}\right)^{l_{j}}\left(\frac{1}{2}-i \eta_{j}\right) \\
& =\sum_{\substack{a_{j}, b_{j}, k_{j}, l_{j} \geq 0 \\
j=1, \ldots, d \\
\operatorname{Tr}((a+1) k+(b+1) l) \leq 3+d}} d(\underline{a}, \underline{k} ; \underline{b}, \underline{l}) \prod_{j=1}^{d}\left(\psi^{\left(a_{j}\right)}\right)^{k_{j}}\left(\psi^{\left(b_{j}\right)}\right)^{l_{j}}\left(\frac{1}{2}-i \eta_{j}\right) .
\end{aligned}
$$

Using the asymptotic formulas of the Polygamma functions

$$
\begin{aligned}
\psi^{(0)}(z) & =\log (z)+\mathcal{O}\left(\frac{1}{z}\right), \\
\psi^{(n)}(z) & =\frac{1}{z}+\mathcal{O}\left(\frac{1}{z^{2}}\right),
\end{aligned}
$$

we obtain the asymptotic

$$
\begin{aligned}
\sum_{\substack{a_{j}, b_{j}, k_{j}, l_{j} \geq 0 \\
j=1, \ldots, d \\
\operatorname{Tr}(a k+b l) \leq 3+d}} d(\underline{a}, \underline{k} ; \underline{b}, \underline{l}) \prod_{j=1}^{d}\left(\frac{\Gamma^{\left(a_{j}\right)}}{\Gamma}\right)^{k_{j}}\left(\frac{\Gamma^{\left(b_{j}\right)}}{\Gamma}\right)^{l_{j}}\left(\frac{1}{2}-i \eta_{j}\right) \\
=p_{3+d}\left(\log \left(N\left(\frac{1}{2}-i \underline{\eta}\right)\right)\right)+\mathcal{O}\left(\left(1+\min _{j}\left|\eta_{j}\right|\right)^{-1}\right)
\end{aligned}
$$

where $N\left(\frac{1}{2}-i \underline{\eta}\right)=\left(\frac{1}{2}-i \eta_{1}\right) \cdots\left(\frac{1}{2}-i \eta_{d}\right)$ and $p_{n}$ is a polynomial of degree $n$ whose coefficients are real constants that can be made explicit. 
Inserting this asymptotic in 3.3.6, we obtain

$$
\begin{aligned}
& \mathcal{J}^{(r)}\left(p_{\frac{1}{2}} ; g, \omega\right)=\mathcal{O}\left(T^{\frac{d}{2}+\varepsilon}\right) \\
& \quad+\sum_{\underline{\nu} \in \Lambda^{d-1}} \Omega(\underline{\nu} / T) \int \ldots \int \widehat{\mathrm{p}}^{2}(\underline{\xi} / \bar{\nu}) \int_{\mathbb{R}^{d-1}}^{\infty} G\left(\frac{t}{T}\right)\left[p_{3+d}\left(\log \left(N\left(\frac{1}{2}-i \underline{\eta}\right)\right)\right)+\mathcal{O}\left(\frac{1}{1+\min _{j}\left|\eta_{j}\right|}\right)\right] d t d \underline{\xi} .
\end{aligned}
$$

Note that $\underline{\eta} \in \mathbb{R}^{d}$ so that the argument of the logarithm is bounded away from zero.

We remark that in every hypercube that is a fundamental domain of the lattice $\Lambda^{d-1}$ there are $T^{d-1}$ elements such that $T \underline{\nu} \in \Lambda^{d-1}$. Let $\underline{\nu} \in \Lambda^{d-1}$, then the points $\underline{\nu}+\bar{\nu} \underline{k} / T$ where $0 \leq k_{j} \leq T-1$ and $\bar{\nu}$ is the basis element of $\Lambda$ defined in (3.1.4) are contained in a fundamental domain of $\Lambda^{d-1}$. Moreover, by (3.3.1) we get $\Omega(\underline{\nu}+\bar{\nu} \underline{k} / T)=\Omega(\underline{\nu})+\mathcal{O}\left(\frac{1}{T}(1+\operatorname{Tr}|\underline{\nu}|)^{-A}\right)$. Thus, substituting $\frac{t}{T} \rightarrow x$ and $\nu_{j} / T \rightarrow y_{j}$, the second line of 3.3 .12 changes to

$$
\begin{gathered}
T^{d} \sum_{\underline{y} \in \Lambda^{d-1}}\left[\Omega(\underline{y})+\mathcal{O}\left(\frac{1}{T}(1+\operatorname{Tr}|\underline{y}|)^{-A}\right)\right] \int_{-\infty}^{\infty} G(x) \int \ldots \int \widehat{\mathrm{p}}^{2}(\underline{\xi} / \bar{\nu}) \times \\
\times\left[p_{3+d}\left(\log \left(N\left(\frac{1}{2}-i \underline{\eta}\right)\right)\right)+\mathcal{O}\left(\left(1+\min _{j}\left|\eta_{j}\right|\right)^{-1}\right)\right] d \underline{\xi} d x
\end{gathered}
$$

where $\eta_{j}=T\left(x+\frac{\xi_{j}}{T}-y_{j}\right)$ for $j=1, \ldots, d-1$ and $\eta_{d}=T(t-\operatorname{Tr}(\underline{\xi}) / T+\operatorname{Tr}(\underline{y}))$.

The function $\widehat{\mathrm{p}}(\underline{\xi})$ is compactly supported in $\left(-\frac{\pi}{2}, \frac{\pi}{2}\right)^{d-1}$. It follows that the summand $\xi_{j} / T$ is relatively small in comparison to $x$ and $y$ and the integral over $\underline{\xi}$ is of constant size.

The polynomial $p_{3+d}\left(\log \left(N\left(\frac{1}{2}-i \underline{\eta}\right)\right)\right)$ can be considered as a sum of a polynomial $P_{3+d}(\log T)$ whose coefficients are polynomials in $\log (N(x-\underline{y}))$ of degree at most $3+d$ that are independent of $T$ and an error term $\mathcal{O}\left((\log N(x-\underline{y}))^{3+d} / T\right)$. This can be seen by applying the equation $\log \left(N(T(x-\underline{y}))^{k}=\right.$ $(d \log (T)+\log N(x-\underline{y}))^{k}$. More precisely the $k$-th coefficient of $P_{3+d}$ is a polynomial in $\log (N(x-\underline{y}))$ of degree at most $3+d-k$.

Thus, 3.3.13 equals

$$
T^{d} \sum_{k=0}^{3+d} C(\mathrm{p}, d) \sum_{\underline{y} \in \Lambda^{d-1}} \Omega(\underline{y}) \int_{-\infty}^{\infty} G(x) P(\log N(x-\underline{y})) d x \cdot(\log T)^{k}+\mathcal{O}\left(T^{d-1+\varepsilon}\right)
$$

where $P$ is a polynomial of degree at most $3+d$ and $C(\mathrm{p}, d)$ are the constants coming from the coefficients $d(\cdot, \cdot ; \cdot, \cdot)$ and the integral over $\underline{\xi}$.

Using the estimates for $G$ and $\Omega$ given in (3.3.1), we see that the sum over $\underline{y}$ and the integral over $x$ are absolutely convergent, and we obtain

$$
\mathfrak{J}^{(r)}\left(p_{\frac{1}{2}} ; g, \omega\right)=T^{d} P_{3+d}(\log T)+\mathcal{O}\left(T^{d-1+\varepsilon}\right)
$$

Thus, combining the asymptotic formula for $\mathcal{J}^{(r)}\left(p_{\frac{1}{2}} ; g, \omega\right)$ with the estimate of $C_{K}$ in 3.3 .9 we get

$$
M_{K}(g, \omega)=T^{d} P_{3+d}(\log T)+\mathcal{O}\left(T^{d-1+\varepsilon}\right) .
$$

Combining the result 3.3.5) of the Section 3.3.1 and 3.3.14 of Section 3.3.2 we obtain the proof of Theorem 1.1.2. 


\section{Chapter 4}

\section{Second Moment of Rankin-Selberg $L$-functions over Totally Real Number Fields}

In this chapter, we prove Theorem 1.2.1. To this end, we need some further results and definitions.

\subsection{Preliminaries}

Recall that $K$ is a totally real number field of degree $d$ and class number one.

\subsubsection{Hilbert Modular Forms}

Let $\mathfrak{c}$ be an integral ideal of $K$. Define

$$
\begin{aligned}
& \Gamma_{0}(\mathfrak{c})=\left\{\left(\begin{array}{cc}
a_{\gamma} & b_{\gamma} \\
c_{\gamma} & d_{\gamma}
\end{array}\right) \in P G L_{2}(\mathfrak{o}) \mid a_{\gamma} d_{\gamma}-b_{\gamma} c_{\gamma} \succ 0, c_{\gamma} \in \mathfrak{c}\right\} \\
& \Gamma_{1}(\mathfrak{c})=\left\{\left(\begin{array}{cc}
a_{\gamma} & b_{\gamma} \\
c_{\gamma} & d_{\gamma}
\end{array}\right) \in P G L_{2}(\mathfrak{o}) \mid a_{\gamma} d_{\gamma}-b_{\gamma} c_{\gamma} \succ 0, a_{\gamma} \equiv d_{\gamma} \equiv 1 \bmod \mathfrak{c}, c_{\gamma} \in \mathfrak{c}\right\}
\end{aligned}
$$

We identify a matrix $\gamma \in G L_{2}(\mathfrak{o})$ with its corresponding vector $\left(\gamma_{1}, \ldots, \gamma_{d}\right) \in G L_{2}(\mathbb{R})^{d}$, where the components are given by $\gamma_{j}=\left(\begin{array}{cc}a_{j} & b_{j} \\ c_{j} & d_{j}\end{array}\right)$ and $a_{j}, b_{j}, c_{j}, d_{j}$ are the $j$-th embeddings of $a_{\gamma}, b_{\gamma}, c_{\gamma}, d_{\gamma} \in \mathfrak{o}$. Then, a matrix $\gamma \in G L_{2}(\mathfrak{o})$ with $\operatorname{det} \gamma \succ 0$ acts on the $d$-fold product $\mathbb{H}^{d}$ via the Möbius transform $\gamma \underline{z}=\left(\frac{a_{j} z_{j}+b_{j}}{c_{j} z_{j}+d_{j}}\right)_{j=1, \ldots, d}$.

A Hilbert modular form $f$ of weight $\underline{k} \in \mathbb{N}_{0}^{d}$ and level $\mathfrak{c}$ is a holomorphic function $f: \mathbb{H}^{d} \rightarrow \mathbb{C}$ satisfying

$$
\left.f\right|_{\gamma}(\underline{z}):=\prod_{j=1}^{d}\left(\frac{\left(\operatorname{det} \gamma_{j}\right)^{\frac{1}{2}}}{c_{j} z_{j}+d_{j}}\right)^{k_{j}} f(\gamma \underline{z})=f(\underline{z})
$$

for all $\gamma \in \Gamma_{0}(\mathfrak{c})$.

If $f$ vanishes at all cusps, it is called a cusp form. We denote the space of cusp forms of weight $\underline{k}$ and level $\mathfrak{c}$ by $S_{\underline{k}}(\mathfrak{c})$ and a basis of it by $\mathcal{B}_{\underline{k}}(\mathfrak{c})$. We may assume that all elements of $\mathcal{B}_{\underline{k}}(\mathfrak{c})$ are Hecke eigenfunctions, since these functions generate the space of cusp forms. 
We define the inner product for two modular forms $f_{1}, f_{2}$ of weight $\underline{k}$ and level $\mathfrak{c}$ by

$$
\left\langle f_{1}, f_{2}\right\rangle=\int_{\Gamma_{0}(\mathfrak{c}) \backslash \mathbb{H}^{d}} f_{1}(\underline{z}) \bar{f}_{2}(\underline{z}) \prod_{j=1}^{d} y_{j}^{k_{j}} \frac{d x_{j} d y_{j}}{y_{j}^{2}} .
$$

As usual $\underline{z}=\left(z_{1}, \ldots, z_{d}\right)=\left(x_{1}+i y_{1}, \ldots, x_{d}+i y_{d}\right)$.

It is well known that $\operatorname{dim} S_{\underline{k}}(\mathfrak{c})$ is finite, see [L03, page 130], more precisely, it is of size $N(\underline{k} \mathfrak{c})^{1+o(1)}$.

Any $f \in S_{\underline{k}}(\mathfrak{c})$ has a Fourier expansion

$$
f(\underline{z})=\sum_{\substack{n \in \mathfrak{o} \\ n \succ 0 \\ \mathfrak{n}=(n)}} C_{f}(\mathfrak{n})(N \mathfrak{n})^{\frac{k-1}{2}} e\left[n \delta^{-1} \underline{z}\right]
$$

where $\mathfrak{d}=(\delta)$ with $\delta \succ 0$ is the different of $K$.

We denote by $\mathcal{B}_{\underline{k}}(\mathfrak{c})$ an orthogonal basis of $S_{\underline{k}}(\mathfrak{c})$ consisting of Hecke eigenfunctions. The Fourier coefficients of $f \in \mathcal{B}_{\underline{k}}(\mathfrak{c})$, normalized by $C_{f}(1)=1$ are real and bounded by

$$
C_{f}(\mathfrak{n}) \ll_{\varepsilon}(N \mathfrak{n})^{\varepsilon},
$$

see [HT16, equation (2.2), Section 6.3] and [LM14, equation (2.1)].

We will consider a subspace of the cusp forms, the so called newforms $S_{\underline{k}}^{\sharp}(\mathfrak{c})$. This space is defined as the orthogonal complement with respect to the inner product of the oldforms where the oldforms $S_{\underline{k}}^{b}(\mathfrak{c})$ are the cusp forms coming from lower level, see [HT16, page 4].

A basis of newforms can be given by primitive forms, i.e. Hecke eigenfunctions that are moreover newforms. We denote a basis of newforms consisting of primitive forms by $\mathcal{B}_{k}^{\sharp}(\mathfrak{c})$.

Let $f$ be a primitive form, then it follows by the work of Shimura [S78, (2.26)] that its Fourier coefficient $C_{f}(\mathfrak{n})$ is equal to the Hecke eigenvalue of the Hecke operator $T_{\mathfrak{n}}$ as defined in (3.1.34).

\subsubsection{Atkin-Lehner Theory}

We give a short overview of the Atkin-Lehner theory, following [AL78] and [KMV00, A3].

Let $\mathfrak{q}=(q)$ be an ideal and let $g \in S_{\underline{\underline{k}}}^{\sharp}(\mathfrak{q})$ with the Fourier coefficients $C_{g}(\cdot)$.

Then we write $q=q_{1} q_{2}$ with $\left(q_{1}, q_{2}\right)=1$. Define for $x, y, z, w \in \mathfrak{o}$ with $x \equiv 1\left(\bmod q_{2}\right), y \equiv 1\left(\bmod q_{1}\right)$ and $q_{1}^{2} x w-q y z=q_{1}$ the matrix

$$
W_{q_{1}}=\left(\begin{array}{cc}
x q_{1} & y \\
z q & w q_{1}
\end{array}\right) \in G L_{2}(\mathfrak{o}) .
$$

A short computation shows that $W_{q_{1}}$ normalizes $\Gamma_{0}(\mathfrak{q})$ and $\Gamma_{1}(\mathfrak{q})$. Furthermore one easily sees that $W_{q_{1}}^{2}$ is the identity, as $W_{q_{1}}^{2} \in \Gamma_{0}(\mathfrak{q})$. Thus $W_{q_{1}}$ defines an endomorphism of $S_{k}^{\sharp}(\mathfrak{q})$, which is independent of the choice of $x, y, z$ and $w$. This endomorphism is given by

$$
\left.g\right|_{W_{q_{1}}}(\cdot)=\eta_{g}\left(q_{1}\right) g(\cdot)
$$

with some constant $\eta_{g}\left(q_{1}\right)$ that satisfies $\left|\eta_{g}\left(q_{1}\right)\right|=1$ as follows from $W_{q_{1}}^{2} \in \Gamma_{0}(\mathfrak{q})$. 
The eigenvalue $\eta_{g}$ can be given explicitly in terms of $C_{g}$ :

\section{(4.1.1) Lemma}

Let $q \in \mathfrak{o}, \mathfrak{q}=(q)$, take a newform $g$ as above and assume $C_{g}(\mathfrak{q}) \neq 0$. Then the eigenvalue $\eta_{g}(q)$ of $W_{q}$ is given by

$$
\eta_{g}(q)=\frac{-1}{C_{g}(\mathfrak{q}) \sqrt{N q}}
$$

Proof.

The proof follows the paper of Atkin and Li [AL78, Theorem 2.1].

We may assume $W_{q}$ to be such that $x \equiv 0(\bmod q)$. Further, for each $u \bmod q$ with $(u, q)=1$ there exists a unique $v \bmod q$ with $(v, q)=1$ such that $u z v \equiv y(\bmod q)$.

For such pairs $(u, v)$, we obtain by a simple multiplication that

$$
\left(\begin{array}{ll}
1 & u \\
0 & q
\end{array}\right) \cdot W_{q} \cdot\left(\begin{array}{cc}
1 & -v / q \\
0 & 1
\end{array}\right) \in \Gamma_{0}(\mathfrak{q})
$$

Thus, we get

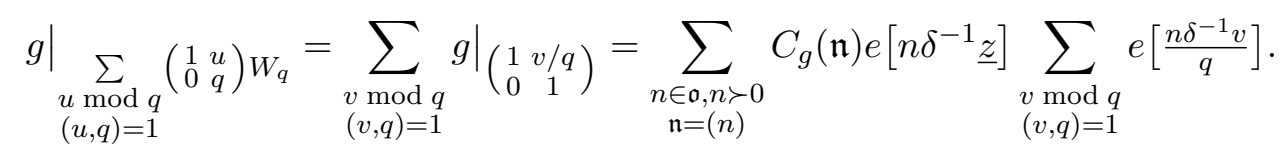

Rewriting this sum over $v$, the right hand side becomes

$$
\left.\left.N(q-1) g\right|_{U_{q}}\right|_{B_{q}}-\sum_{\substack{n \in \mathfrak{o}, n \succ 0 \\ \mathfrak{n}=(n)}} C_{g}(\mathfrak{n}) e\left[n \delta^{-1} \underline{z}\right]
$$

where

$$
\begin{aligned}
\left.g\right|_{U_{q}}(\underline{z}) & =\sum_{\substack{n \in \mathfrak{o}, n \succ 0 \\
\mathfrak{n}=(n)}} C_{g}(\mathfrak{q n}) e\left[n \delta^{-1} \underline{z}\right]=\left.(N q)^{-\frac{1}{2}} \sum_{u \bmod q} g\right|_{\left(\begin{array}{ll}
1 & u \\
0 & q
\end{array}\right)}(\underline{z}) \\
\text { and }\left.g\right|_{B_{q}}(\underline{z}) & =\sum_{\substack{n \in \mathfrak{o}, n \succ 0 \\
\mathfrak{n}=(n)}} C_{g}(\mathfrak{n}) e\left[q n \delta^{-1} \underline{z}\right]=g(q \underline{z}) .
\end{aligned}
$$

Now we view $\left.\left.g\right|_{U_{q}}\right|_{W_{q}}$ in two ways. First, we insert the definitions and reformulate the sums, to get

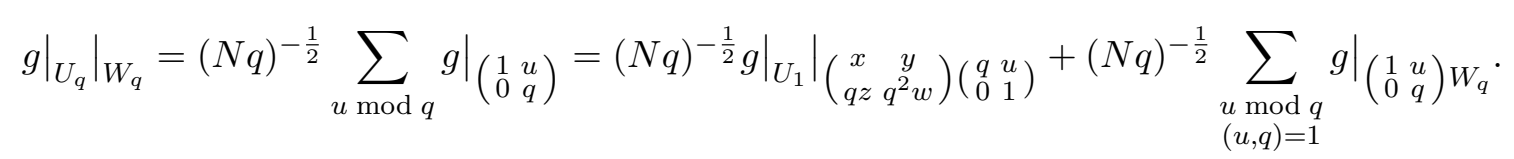

Now, we insert 4.1.4 for the second summand, to obtain

$$
\left.\left.g\right|_{U_{q}}\right|_{W_{q}}=\left.\left.(N q)^{-\frac{1}{2}} g\right|_{U_{1}}\right|_{\left(\begin{array}{cc}
x & y \\
q z & q^{2} w
\end{array}\right)\left(\begin{array}{cc}
q & u \\
0 & 1
\end{array}\right)}+\left.\left.N(q-1)(N q)^{-\frac{1}{2}} g\right|_{U_{q}}\right|_{B_{q}}-(N q)^{-\frac{1}{2}} \sum_{\substack{n \in \mathfrak{o}, n \succ 0 \\
\mathfrak{n}=(n)}} C_{g}(\mathfrak{n}) e\left[n \delta^{-1} \underline{z}\right] .
$$


On the other hand we have

$$
\left.\left.g\right|_{U_{q}}\right|_{W_{q}}=C_{g}(\mathfrak{q}) \eta_{g}(q) \cdot g
$$

Thus, comparing the first Fourier coefficients ends the proof.

We need the following result in the proof of a Voronoi summation formula in Section 4.1.4

\section{(4.1.2) Lemma}

Let $q \in \mathcal{F}, \mathfrak{q}=(q), a, c \in \mathfrak{o}$ with $(a, c)=1$. Let $D_{1}=(c, q), D_{2}=\frac{q}{D_{1}}=\frac{q}{(c, q)} \in \mathcal{F}$ and suppose that $D_{1}$ and $D_{2}$ are coprime.

Then, there exists a matrix $\gamma=\left(\begin{array}{ll}a & b \\ c & d\end{array}\right) \in S L_{2}(\mathfrak{o})$, such that the following identity holds (as operators acting on weight $\underline{k}$ modular forms)

$$
\gamma=\left(\begin{array}{ll}
a & b \\
c & d
\end{array}\right)=\left(\begin{array}{cc}
a^{\prime} & b^{\prime} \\
c^{\prime} q & d^{\prime}
\end{array}\right) \circ W_{D_{2}} \circ\left(\begin{array}{cc}
1 & 0 \\
0 & D_{2}
\end{array}\right)
$$

for some $\gamma_{1}=\left(\begin{array}{cc}a^{\prime} & b^{\prime} \\ c^{\prime} q & d^{\prime}\end{array}\right) \in \Gamma_{0}(\mathfrak{q})$. Moreover $d^{\prime}$ satisfies the congruences

$$
d^{\prime} \equiv a^{*} \quad\left(\bmod D_{1}\right), \quad d^{\prime} \equiv-c \quad\left(\bmod D_{2}\right)
$$

where $a^{*}$ is the multiplicative inverse of a modulo $D_{1}$.

Proof.

The proof is analogous to [KMV00, Lemma A.3].

Since $(a, c)=1$ and $\left(c, q, D_{2}\right)=1$, we may choose the lower right entry $d$ of $\gamma$ such that $D_{2} \mid d$. Then pick any matrix $W_{D_{2}}=\left(\begin{array}{cc}x D_{2} & y \\ z q & w D_{2}\end{array}\right)$ representing the Atkin-Lehner operator. Recall that $y \equiv 1\left(\bmod D_{2}\right)$ and $x \equiv 1\left(\bmod D_{1}\right)$, as well as $\operatorname{det} W_{D_{2}}=D_{2}$.

Then we get

$$
\gamma_{1}:=D_{2} I \cdot \gamma \cdot \operatorname{diag}\left(1, D_{2}^{-1}\right) \cdot W_{D_{2}}^{-1}=\left(\begin{array}{ll}
a w D_{2}-z b D_{1} & -a y+b x \\
c w D_{2}-z d D_{1} & -c y+d x
\end{array}\right) \in \Gamma_{0}(\mathfrak{q})
$$

by our choice of $d$. The congruences 4.1.5 follow from the congruences of $x$ and $y$.

\subsubsection{Rankin-Selberg $L$-functions}

Now we are able to define the Rankin-Selberg $L$-function and state some results. We follow the terminology used in [HT16] and [L79].

Let $\mathfrak{q}=(q)$ be prime and $\mathfrak{c}$ a squarefree integral ideal either coprime to $\mathfrak{q}$ or equal to $\mathfrak{q}$. Further, let $f \in S_{\underline{k}}^{\sharp}(\mathfrak{c}), g \in S_{\underline{k}}^{\sharp}(\mathfrak{q})$ be two primitive forms of weight $\underline{k} \in \mathbb{N}^{d}$. Denote by $C_{f}(\mathfrak{n}), C_{g}(\mathfrak{n})$ their Fourier coefficients.

Then, the Rankin-Selberg $L$-function is, for $\operatorname{Re} s>1$, defined by

$$
L(f \otimes g, s)=L\left(2 s, \chi_{\mathfrak{q} \mathfrak{c}}\right) \sum_{\mathfrak{n}} \frac{C_{f}(\mathfrak{n}) C_{g}(\mathfrak{n})}{(N \mathfrak{n})^{s}}
$$


where $\chi_{\mathfrak{q c}}$ is the trivial character modulo $\mathfrak{q} \mathfrak{c}$ given by

$$
\begin{aligned}
\chi_{\mathfrak{q} \mathfrak{c}}(\mathfrak{n}) & = \begin{cases}1, & (\mathfrak{n}, \mathfrak{q} \mathfrak{c})=1 \\
0, & (\mathfrak{n}, \mathfrak{q} \mathfrak{c})>1\end{cases} \\
\text { and } \quad L\left(2 s, \chi_{\mathfrak{q} \mathfrak{c}}\right) & =\sum_{\mathfrak{n}} \frac{\chi_{\mathfrak{q} \mathfrak{c}}(\mathfrak{n})}{(N \mathfrak{n})^{2 s}}=\prod_{\substack{\mathfrak{p} \mid \mathfrak{q} \mathfrak{c} \\
\mathfrak{p p r i m e}}}\left(1-(N \mathfrak{p})^{-2 s}\right) \zeta_{K}(2 s)
\end{aligned}
$$

The Rankin-Selberg $L$ functions satisfies a functional equation:

\section{(4.1.3) Lemma}

Let $\underline{k} \in \mathbb{N}^{d}$ and $f, g$ be two primitive forms with $f \in S_{\underline{k}}^{\sharp}(\mathfrak{c}), g \in S_{\underline{k}}^{\sharp}(\mathfrak{q})$ where $\mathfrak{q}$ is a prime ideal and either $(\mathfrak{c}, \mathfrak{q})=1$ or $\mathfrak{c}=\mathfrak{q}$. Furthermore, let $C_{g}(\mathfrak{q}) \neq 0$ and $C_{f}((\mathfrak{q}, \mathfrak{c})) \neq 0$.

Then, the Rankin-Selberg L-function satisfies

$$
\begin{aligned}
\Lambda(f \otimes g, s): & =(2 \pi)^{-2 s d}\left(N \frac{\mathfrak{q} \mathfrak{c}}{(\mathfrak{q}, \mathfrak{c})}\right)^{s}\left|D_{K}\right|^{2 s} L_{\infty}(f \otimes g, s) \prod_{\mathfrak{p} \mid(\mathfrak{q}, \mathfrak{c})} \theta_{\mathfrak{p}}(s)^{-1} L(f \otimes g, s) \\
& =\Lambda(f \otimes g, 1-s)
\end{aligned}
$$

where

$$
\begin{aligned}
& L_{\infty}(f \otimes g, s)=\prod_{j=1}^{d}(2 \pi)^{-k_{j}} \Gamma(s) \Gamma\left(s+k_{j}-1\right), \\
& \theta_{\mathfrak{p}}(s)= \begin{cases}1-C_{f}(\mathfrak{p}) C_{g}(\mathfrak{p})(N \mathfrak{p})^{1-s}, & \text { if } \mathfrak{p}=\mathfrak{c}=\mathfrak{q}, \\
1, & \text { else. }\end{cases}
\end{aligned}
$$

Proof.

The case that $\mathfrak{c}$ and $\mathfrak{q}$ are coprime is treated in [HT16]. Thus, we assume $\mathfrak{c}=\mathfrak{q}=(c), c \in \mathcal{F}$ and follow the paper of $\mathrm{Li}[\mathrm{L} 79 \mid$.

Let

$$
h(\underline{z}, s, a)=\sum_{\substack{(\mathfrak{m} \mathfrak{c}, \mathfrak{n})=1 \\ \mathfrak{n}=(n), n \in \mathcal{F} \\ n \equiv a \bmod c}} \underline{y}^{s}(N|m c+n|)^{-2 s}
$$

Writing out the Fourier expansions of $f$ and $g$ and using orthogonality of characters as well as an unfolding argument to obtain

$$
\frac{1}{\varphi(\mathfrak{c})} \int \ldots \int f(\underline{z}) \bar{g}(\underline{z}) \sum_{D_{1}(\mathfrak{c})} \chi_{a \in \mathcal{F}}(a) h(\underline{z}, s, a) d \mu(\underline{z})=(4 \pi)^{-d s-(\underline{k}-1)} \Gamma(s+\underline{k}-1) \sum_{\mathfrak{n}} \frac{C_{f}(\mathfrak{n}) C_{g}(\mathfrak{n})}{(N \mathfrak{n})^{s}} .
$$

The product of the sum over $a$ with $(N \mathfrak{c})^{s}$ and $L\left(2 s, \chi_{\mathfrak{c}}\right)$ equals a sum over the Eisenstein function. More precisely

$$
(N \mathfrak{c})^{s} L\left(2 s, \chi_{\mathfrak{c}}\right) \sum_{a \bmod c} \chi_{\mathfrak{c}}(\mathfrak{a}) \sum_{\substack{(\mathfrak{m} \mathfrak{s}, \mathfrak{n})=1 \\ \mathfrak{n}=(n), n \in \mathcal{F} \\ n \equiv a \bmod c}} \underline{y}^{s}(N|m c+n|)^{-2 s}=\sum_{\substack{a \mid c \\ a \in \mathcal{F}}} \mu((a))(N a)^{-s} E(c \underline{z} / a, s)
$$


where $\mu(\mathfrak{a})$ is the Möbius function.

Recall that $\Gamma(s+\underline{k}-1)=\prod_{j=1}^{d} \Gamma\left(s+k_{j}-1\right)$. We get

$$
\begin{aligned}
\Phi_{f g}(s): & =(N \mathfrak{c})^{s} \pi^{-d s}(4 \pi)^{-d s-(\underline{k}-1)} \Gamma(s+\underline{k}-1) \Gamma(s)^{d} L(f \otimes g, s) \\
& =\varphi(\mathfrak{c})^{-1} \pi^{-d s} \Gamma(s)^{d} \sum_{a \mid c} \mu(a)(N a)^{-s} \int_{D_{1}(\mathfrak{c})} \ldots \int f(\underline{z}) \bar{g}(\underline{z}) E(c \underline{z} / a, s) d \mu(\underline{z}) .
\end{aligned}
$$

Since $c$ is prime, the sum over $a$ contains only two summands, $a=1$ and $a=c$. Our aim is to reduce the term $a=c$ to $a=1$ with an additional constant in front of the integral.

Let $\mathfrak{p}=(p) \mid \mathfrak{c}$. Now recall that the Atkin-Lehner operator $W_{p}$ normalizes $\Gamma_{1}(\mathfrak{c})$.

Furthermore $E(c \underline{z} / a p, s) \circ W_{p}=E(c \underline{z} / a, s)$, and we have

$$
\begin{aligned}
\int \ldots \int f(\underline{z}) \bar{g}(\underline{z}) E(c \underline{z} / a p, s) d \mu(\underline{z}) & =\eta_{f}(p) \bar{\eta}_{g}(p) \int \ldots \int f(\underline{z}) \bar{g}(\underline{z}) E(c \underline{z} / a, s) d \mu(\underline{z}) \\
& =C_{D_{1}(\mathfrak{c})}(\mathfrak{p}) C_{g}(\mathfrak{p}) N \mathfrak{p} \int_{D_{1}(\mathfrak{c})} \ldots \int f(\underline{z}) \bar{g}(\underline{z}) E(c \underline{z} / a, s) d \mu(\underline{z}) .
\end{aligned}
$$

The second equality follows from Lemma 4.1.1 and the fact that $C_{f}$ and $C_{g}$ are real and non-zero by our assumption.

Now we get

$$
\sum_{\substack{a \mid c \\ a \in \mathcal{F}}} \mu((a))(N a)^{-s} \int \ldots \int f(\underline{z}) \bar{g}(\underline{z}) E(c \underline{z} / a, s) d \mu(\underline{z})=\theta_{\mathfrak{p}}(s) \int \ldots \int f(\underline{z}) \bar{g}(\underline{z}) E(c \underline{z}, s) d \mu(\underline{z})
$$

where

$$
\theta_{\mathfrak{p}}(s)=1-C_{f}(\mathfrak{p}) C_{g}(\mathfrak{p})(N \mathfrak{p})^{1-s}
$$

Thus the functional equation of $\Phi_{f g}(s)$ is induced by the functional equation of the Eisenstein series which can be stated as

$$
\pi^{-d s} \Gamma(s)^{d} E(c \underline{z} / a, s)=\pi^{-d(1-s)} \Gamma(1-s)^{d} E(c \underline{z} / a, 1-s) .
$$

This follows from Lemma 3.1 .2 by replacing $s$ with $s-\frac{1}{2}$.

Finally the functional equation of $\Phi_{f g}(s)$ yields the result for $\Lambda(f \otimes g, s)$.

We define the multiplicative coefficients $\gamma_{f g}(\mathfrak{b})$ by

$$
\sum_{\mathfrak{b} \mid(\mathfrak{q}, \mathfrak{c})^{\infty}} \gamma_{f g}(\mathfrak{b})(N \mathfrak{b})^{-s}:=\prod_{\mathfrak{p} \mid(\mathfrak{q}, \mathfrak{c})} \theta_{\mathfrak{p}}(s)^{-1}
$$

Note, for $\mathfrak{p} \mid(\mathfrak{q}, \mathfrak{c})$, that $\left|\theta_{\mathfrak{p}}(s)\right| \geq\left(1-(N \mathfrak{p})^{-\sigma}\right)$ as $\left|C_{f}(\mathfrak{p})\right|=\sqrt{N \mathfrak{p}}^{-1}$ which follows from Lemma 4.1.1 and $\left|\eta_{f}(p)\right|=1$. Thus, multiplying out the product $\prod_{\mathfrak{p} \mid(\mathfrak{q}, \mathfrak{c})}\left(1-(N \mathfrak{p})^{-\sigma}\right)^{-1}$ we obtain in the case $\operatorname{Re} s=\sigma>\varepsilon$ absolute convergence for any $\varepsilon>0$. Comparing the left hand side of 4.1.9) with the 
bound of the right hand side of 4.1.9) we obtain for any $\varepsilon>0$ :

$$
\left|\gamma_{f g}(\mathfrak{b})\right| \ll(N \mathfrak{b})^{\varepsilon}
$$

Using the functional equation of the Rankin-Selberg $L$-function, we obtain the approximate functional equation

\section{(4.1.4) Lemma}

Let $\mathfrak{q}$ be a prime ideal and $\mathfrak{c}$ an integral ideal either coprime or equal to $\mathfrak{q}$. Let $f \in S_{\underline{k}}^{\sharp}(\mathfrak{c}), g \in S_{\underline{k}}^{\sharp}(\mathfrak{q})$, with $C_{g}(\mathfrak{q}) \neq 0$ and $C_{f}(\mathfrak{q}) \neq 0$. Further define

$$
V_{s}(y):=\frac{1}{2 \pi i} \int_{\left(\frac{3}{2}\right)} y^{-u} \prod_{j=1}^{d} \frac{\Gamma(s+u) \Gamma\left(s+u+k_{j}-1\right)}{\Gamma(s) \Gamma\left(s+k_{j}-1\right)} G(u) \frac{d u}{u}
$$

where $G(u)$ is a holomorphic function on an open set containing the strip $|\operatorname{Re}(u)| \leq \frac{3}{2}$ and bounded therein, satisfying $G(u)=G(-u)$ and $G(0)=1$.

Then we have

$$
L\left(f \otimes g, \frac{1}{2}\right)=2 \sum_{\mathfrak{b} \mid(\mathfrak{q}, \mathfrak{c})^{\infty}} \frac{\gamma_{f g}(\mathfrak{b})}{(N \mathfrak{b})^{\frac{1}{2}}} \sum_{\mathfrak{a}} \frac{\chi_{\mathfrak{q} \mathfrak{c}}(\mathfrak{a})}{(N \mathfrak{a})} \sum_{\mathfrak{m}} \frac{C_{f}(\mathfrak{m}) C_{g}(\mathfrak{m})}{(N \mathfrak{m})^{\frac{1}{2}}} V_{\frac{1}{2}}\left(\frac{(2 \pi)^{2 d} N\left(\mathfrak{b} \mathfrak{a}^{2} \mathfrak{m}\right)}{D_{K}^{2} N\left(\frac{\mathfrak{q} \mathfrak{c}}{(\mathfrak{q}, \mathfrak{c})}\right)}\right) .
$$

Moreover, the derivatives of $V_{\frac{1}{2}}(y)$ satisfy

$$
y^{\nu} V_{\frac{1}{2}}^{(\nu)}(y) \ll_{\nu, A}\left(1+\frac{y}{N \underline{k}}\right)^{-A}
$$

for all $\nu \in \mathbb{N}_{0}$.

Proof.

See [LM14, Proposition 3.2] and [HT16, Proposition 4.1].

\subsubsection{Voronoi's Formula}

Later on we want to apply a Voronoi summation formula. To this end, we prove:

\section{(4.1.5) Theorem}

Let $a, c \in \mathfrak{o}$, with $(a, c)=1$. Further let $\underline{k} \in \mathbb{N}^{d}, \mathfrak{q}=(q)$ be a prime ideal and $g \in S_{\underline{k}}(\mathfrak{q})$ with Fourier coefficients $C_{g}(\mathfrak{n})$. Let $F:[0, \infty)^{d} \rightarrow \mathbb{R}$ be a smooth function rapidly decaying if at least one variable tends to infinity and vanishing if at least one variable is zero.

Then we have

$$
\begin{aligned}
& \sum_{\substack{\mathfrak{n}=(n) \\
n \in \mathcal{F}}} C_{g}(\mathfrak{n}) e\left[n \frac{a}{c}\right] F(n)
\end{aligned}
$$

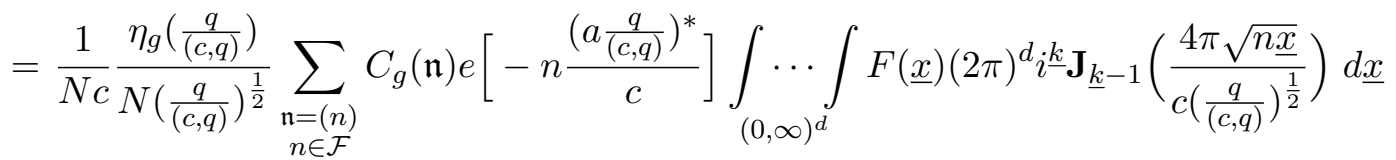


where $\left(a \frac{q}{(c, q)}\right)^{*}$ is the multiplicative inverse modulo $c$ and $\mathbf{J}_{\underline{k}-1}$ is a product of Bessel functions, namely

$$
\mathbf{J}_{\underline{k}-1}(\underline{x})=\prod_{j=1}^{d} J_{k_{j}-1}\left(x_{j}\right)
$$

Proof.

The proof follows KMV00, Appendix A.3]. We will use the Atkin-Lehner operator and the Mellin inversion formula to deduce the Voronoi summation formula.

Let $D_{1}=(c, q) \in \mathfrak{o}$ and $D_{2}=\frac{q}{(c, q)} \in \mathfrak{o}$, and take $\gamma=\left(\begin{array}{ll}a_{\gamma} & b_{\gamma} \\ c_{\gamma} & d_{\gamma}\end{array}\right) \in S L_{2}(\mathfrak{o})$ with $a_{\gamma}=a$ and $c_{\gamma}=c$ as in Lemma 4.1.2, in particular choose $d_{\gamma}$ such that $D_{2} \mid d_{\gamma}$. Let $\underline{z} \in \mathbb{H}^{d}$. Using the factorization of $\gamma$, we have

$$
g(\gamma \underline{z})=\left(c_{\gamma} \underline{z}+d_{\gamma}\right)^{\underline{k}} D_{2}^{-\underline{k} \frac{1}{2}} \eta_{g}\left(D_{2}\right) g\left(\frac{\underline{z}}{D_{2}}\right) .
$$

Now we define $\underline{z_{t}}=\left(-\frac{d_{j}}{c_{j}}+\frac{i}{c_{j} t_{j}}\right)_{j=1, \ldots d}$ for $\underline{t} \in\left(\mathbb{R}_{>0}\right)^{d}$. Thus $c_{\gamma} \underline{z_{t}}+d_{\gamma}=\frac{i}{\underline{t}}$, and $\gamma \underline{z_{t}}=\frac{a_{\gamma}}{c_{\gamma}}+\frac{i \underline{t}}{c_{\gamma}}$. Then by 4.1 .5 we get

$$
e\left[-n \frac{d_{\gamma}}{c_{\gamma} D_{2}}\right]=e\left[-n \frac{d_{\gamma} D_{2}^{*}}{c_{\gamma}}-n \frac{d_{\gamma} c_{\gamma}^{*}}{D_{2}}\right]=e\left[-n \frac{d_{\gamma} D_{2}^{*}}{c_{\gamma}}\right]=e\left[-n \frac{\left(a_{\gamma} D_{2}\right)^{*}}{c_{\gamma}}\right] .
$$

Using this and writing out the definition, we obtain

$$
\begin{gathered}
g\left(\gamma \underline{z_{t}}\right)=\sum_{\mathfrak{n}=(n)} C_{g}(\mathfrak{n}) e\left[n \frac{a_{\gamma}}{c_{\gamma}}\right] n^{\frac{1}{2}(\underline{k}-1)} \exp \left(-\operatorname{Tr}\left(\frac{2 \pi n \underline{t}}{c_{\gamma}}\right)\right) \\
=\left(\frac{i}{\underline{t}}\right)^{\underline{k}} D_{2}^{-\underline{k} \frac{1}{2}} \eta_{g}\left(D_{2}\right) g\left(\frac{\underline{z}}{D_{2}}\right)=\frac{i \underline{\underline{k}} \eta_{g}\left(D_{2}\right)}{\left(N D_{2}\right)^{\frac{1}{2}}} \sum_{\mathfrak{n}=(n)} C_{g}(\mathfrak{n}) e\left[-n \frac{\left(a_{\gamma} D_{2}\right)^{*}}{c_{\gamma}}\right]\left(\frac{n}{D_{2}}\right)^{\frac{1}{2}(\underline{k}-1)} \exp \left(-\operatorname{Tr}\left(\frac{2 \pi n}{c_{\gamma} \underline{t} D_{2}}\right)\right) \underline{t}^{-\underline{k}} .
\end{gathered}
$$

The map $\underline{t} \mapsto g\left(\gamma_{\underline{t}}\right)$ has exponential decay if at least one variable tends to zero or infinity. Thus, the Mellin transform of $g\left(\gamma \underline{z_{t}}\right)$ is analytic on $\mathbb{C}^{d}$. For Re $s_{j}$ sufficiently large for all $j$, by (4.1.13) we obtain

$$
\int_{(0, \infty)^{d}} \cdots \int_{\underline{1}} g\left(\underline{z}_{t}\right) \underline{t}^{\underline{s}+\frac{1}{2}(\underline{k}-1)} \frac{d \underline{t}}{N \underline{t}}=\left(\frac{c_{\gamma}}{2 \pi}\right)^{\underline{s}+\frac{1}{2}(\underline{k}-1)} \Gamma\left(\underline{s}+\frac{1}{2}(\underline{k}-1)\right) \sum_{\mathfrak{n}=(n)} C_{g}(\mathfrak{n}) e\left[n \frac{a_{\gamma}}{c_{\gamma}}\right] n^{-\underline{s}} .
$$

We denote the sum over $\mathfrak{n}$ by $L\left(g, \frac{a_{\gamma}}{c_{\gamma}}, \underline{s}\right)$. Thus $\Gamma\left(\underline{s}+\frac{1}{2}(\underline{k}-1)\right) L\left(g, \frac{a_{\gamma}}{c_{\gamma}}, \underline{s}\right)$ has an analytic continuation to $\mathbb{C}^{d}$.

Multiplying 4.1.13 and 4.1.14 with $\underline{t}^{\underline{s}+\frac{1}{2}(\underline{k}-1)-1}$ and integrating over $\underline{t}$ we have the functional equation

$$
\begin{aligned}
& \left(\frac{c_{\gamma} \sqrt{D_{2}}}{2 \pi}\right)^{\underline{s}} \Gamma\left(\underline{s}+\frac{1}{2}(\underline{k}-1)\right) L\left(g, \frac{a_{\gamma}}{c_{\gamma}}, \underline{s}\right) \\
= & i^{\underline{k}} \eta_{g}\left(D_{2}\right)\left(\frac{c_{\gamma} \sqrt{D_{2}}}{2 \pi}\right)^{1-\underline{s}} \Gamma\left(1-\underline{s}+\frac{1}{2}(\underline{k}-1)\right) L\left(g,-\frac{\left(a_{\gamma} D_{2}\right)^{*}}{c_{\gamma}}, 1-\underline{s}\right) .
\end{aligned}
$$

Note that the left hand side is holomorphic for $\operatorname{Re} \underline{s} \geq \frac{1}{2}(\underline{k}+1)$, and the poles of the $\Gamma$-function on the right hand side are canceled by the zeros of the $L$-function.

Now take $F$ as stated in the Theorem, and denote its Mellin transform by $\widetilde{F}$. Multiplying the functional 
equation 4.1 .15 with $\widetilde{F}$ and integrating over $\underline{s}$ we obtain

$$
\begin{aligned}
& \frac{1}{(2 \pi i)^{d}} \int_{(1) \cdots(1)} \ldots \int_{(1)} \widetilde{F}(\underline{s}) L\left(g, \frac{a_{\gamma}}{c_{\gamma}}, \underline{s}\right) d \underline{s} \\
= & \frac{i \underline{k} \eta_{g}\left(D_{2}\right)}{(2 \pi i)^{d}} \int_{(1) \cdots(1)} \ldots \int_{\int} \widetilde{F}(\underline{s})\left(\frac{c_{\gamma} \sqrt{D_{2}}}{2 \pi}\right)^{1+2} \underline{\underline{s}} \frac{\Gamma\left(1-\underline{s}+\frac{1}{2}(\underline{k}-1)\right)}{\Gamma\left(\underline{s}+\frac{1}{2}(\underline{k}-1)\right)} L\left(g,-\frac{\left(a_{\gamma} D_{2}\right)^{*}}{c_{\gamma}}, 1-\underline{s}\right) d \underline{s} .
\end{aligned}
$$

Then using Mellin inversion the left hand side of 4.1.16 equals

$$
\sum_{\mathfrak{n}=(n)} C_{g}(\mathfrak{n}) e\left[n \frac{a_{\gamma}}{c_{\gamma}}\right] F(n)
$$

To compute the right hand side, we move the contour to $\operatorname{Re} s_{j}=-1$ for all $j$, and change variables $2(1-\underline{s}) \mapsto \underline{s}$. In addition we write out the definition of $L\left(g,-\frac{\left(a_{\gamma} D_{2}\right)^{*}}{c_{\gamma}}, 1-\underline{s}\right)$. Then the sum over $\mathfrak{n}$ is absolutely convergent and the right hand side changes to

$$
\begin{aligned}
& \frac{i \underline{\underline{k}}(2 \pi)^{d} \eta_{g}\left(D_{2}\right)}{N\left(c_{\gamma} \sqrt{D_{2}}\right)} \sum_{\mathfrak{n}=(n)} C_{g}(\mathfrak{n}) e\left[-n \frac{\left(a_{\gamma} D_{2}\right)^{*}}{c_{\gamma}}\right] \times \\
& \times \frac{1}{(4 \pi i)^{d}} \int_{(4) \cdots(4)} \ldots \int_{(4)} \widetilde{F}\left(1-\frac{s}{2}\right) \frac{\Gamma\left(\frac{1}{2}(\underline{s}+\underline{k}-1)\right)}{\Gamma\left(1+\frac{1}{2}(-\underline{s}+\underline{k}-1)\right)}\left(\frac{2 \pi \sqrt{n}}{c_{\gamma} \sqrt{D_{2}}}\right)^{-\underline{s}} d \underline{s} .
\end{aligned}
$$

Finally we write out the Mellin transform $\widetilde{F}$. There is a slight problem of absolute convergence if some $k_{j}=1$, we avoid this by deforming the contour. Now we shift the contours back to $\operatorname{Re} s=\frac{3}{4}$, such that the integral equals

$$
\int_{(0, \infty)^{d}} \ldots \int_{(\underline{x})} F\left(\frac{1}{(4 \pi i)^{d}} \int_{\left(\frac{3}{4}\right) \cdots\left(\frac{3}{4}\right)} \cdots \int_{\Gamma\left(\frac{1}{2}(\underline{s}+\underline{k}-1)\right)} \frac{2 \pi \sqrt{n \underline{x}}}{\Gamma\left(1+\frac{1}{2}(-\underline{s}+\underline{k}-1)\right)}\right)^{-\underline{s}} d \underline{s} d \underline{x} .
$$

Using [GR07, 8.412.4], the inner integrals are a product of Bessel functions and we obtain that the right hand side of 4.1.16 equals

$$
\frac{1}{N c_{\gamma}} \frac{\eta_{g}\left(D_{2}\right)}{N\left(D_{2}\right)^{\frac{1}{2}}} \sum_{\mathfrak{n}=(n)} C_{g}(\mathfrak{n}) e\left[-n \frac{\left(a_{\gamma} D_{2}\right)^{*}}{c_{\gamma}}\right] \int_{(0, \infty)^{d}} \ldots \int_{f} F(\underline{x})(2 \pi)^{d} i^{\underline{k}} \mathbf{J}_{\underline{k}-1}\left(\frac{4 \pi \sqrt{n \underline{x}}}{c_{\gamma} D_{2}^{\frac{1}{2}}}\right) d \underline{x} .
$$

Replacing $D_{2}$ by its definition $\frac{q}{(c, q)}$ and remark that $c_{\gamma}=c$ and $a_{\gamma}=a$ completes the proof.

\subsubsection{Further Results}

A further result we need is the Petersson trace formula. We use the version of Luo [L03, (8)]:

\section{(4.1.6) Lemma (Petersson's trace formula)}

Let $\mathfrak{c} \subseteq K$ be an integral ideal, and $\underline{k} \in \mathbb{N}^{d}$. Let $\mathcal{B}_{\underline{k}}(\mathfrak{c})$ be an orthogonal basis of the cusp forms of weight $\underline{k}$ and level $\mathbf{c}$. Further let $m, n \succ 0$. 
Then we have

$$
\begin{aligned}
& \frac{\Gamma(\underline{k}-1)}{(4 \pi)^{\underline{k}-1}\left|D_{K}\right|^{\frac{1}{2}}} \sum_{f \in \mathcal{B}_{\underline{k}}(\mathfrak{c})} \frac{C_{f}((m)) C_{f}((n))}{\langle f, f\rangle} \\
= & \delta_{m=\epsilon^{2} n}+\frac{(2 \pi)^{d}(-1)^{d \frac{k}{2}}}{\left|D_{K}\right|^{\frac{1}{2}}} \sum_{\epsilon \in \mathfrak{o}^{\times}} \sum_{c \in \mathfrak{c}^{*} / \mathfrak{o}^{\times}} \frac{S_{K}\left(m, \epsilon^{2} n ; c\right)}{|N c|} \mathbf{J}_{\underline{k}-1}\left(\frac{4 \pi \sqrt{m n}|\epsilon|}{|c|}\right)
\end{aligned}
$$

where $\delta_{m=\epsilon^{2} n}=\left\{\begin{array}{ll}1, & \text { if } m=\epsilon^{2} n, \text { for some } \epsilon \in \mathfrak{o}^{\times}, \\ 0, & \text { else }\end{array}\right.$, and $\mathbf{J}_{\underline{k}-1}$ is a product of Bessel functions, namely

$$
\mathbf{J}_{\underline{k}-1}\left(\frac{4 \pi \sqrt{m n}|\epsilon|}{|c|}\right)=\prod_{j=1}^{d} J_{k_{j}-1}\left(\frac{4 \pi \sqrt{m_{j} n_{j}}\left|\epsilon_{j}\right|}{\left|c_{j}\right|}\right) .
$$

The Kloosterman sum $S_{K}(m, n ; c)$ and the inner product are defined in $(2.0 .2)$ and $(4.1 .2)$.

Proof.

See [L03, Section 2].

Further we use the equality from [IL07, equation A.9]

$$
2 \pi J_{k-1}(4 \pi \sqrt{\alpha \gamma}) J_{k-1}(4 \pi \sqrt{\beta \gamma})=\mathcal{K} \int_{0}^{\infty} e((\alpha+\beta) x+\gamma / x) J_{k-1}(4 \pi \sqrt{\alpha \beta} x) \frac{d x}{x}
$$

where $\alpha, \beta, \gamma \geq 0$, and the operator $\mathcal{K}(f)$ is given by $\mathcal{K}(f):=i^{-k} f+i^{k} \bar{f}=2 \operatorname{Re}\left(i^{-k} f\right)$.

By [L03, page 136] we get for $\underline{k}=(k, \ldots, k)$ the estimate

$$
\mathbf{J}_{\underline{k}-1}\left(\frac{4 \pi \sqrt{m n}|\epsilon|}{|c|}\right) \ll \min \left\{1,\left(\frac{(16 e \pi)^{d} \sqrt{N m n}}{k^{d}|N c|}\right)^{k-1}\right\}|N c|^{\eta} \prod_{\left|\epsilon_{j}\right|>1}\left|\epsilon_{j}\right|^{-\eta}
$$

where $0<\eta<\frac{1}{2}$. 


\subsection{Proof of Theorem 1.2 .1}

As before, let $\mathfrak{q}$ be a prime ideal and $\mathfrak{c}$ a squarefree integer ideal in $K$ either coprime or equal to $\mathfrak{q}$ and let $\underline{k}=(k, \ldots, k) \in \mathbb{N}^{d}$. Then for a primitive form $g$ of level $\mathfrak{q}$ and weight $\underline{k}$, with $C_{g}(\mathfrak{q}) \neq 0$, the second moment is given by

$$
M^{\sharp}(g):=\frac{\Gamma(\underline{k}-1)}{(4 \pi)^{\underline{k}-1}\left|D_{K}\right|^{\frac{1}{2}}} \sum_{f \in \mathcal{B}_{\underline{k}}^{\sharp}(\mathfrak{c})} \frac{\left|L\left(f \otimes g, \frac{1}{2}\right)\right|^{2}}{\langle f, f\rangle} .
$$

We will establish an upper bound of this quantity given by $M^{\sharp}(g) \ll\left(N \frac{\mathfrak{q} \mathfrak{c}}{(\mathfrak{q}, \mathfrak{c})}\right)^{\varepsilon} k^{d \varepsilon}$.

\subsubsection{Initial Reduction}

To begin we apply the approximate functional equation given in Lemma 4.1.4. We get

$$
\begin{aligned}
M^{\sharp}(g)=4 \frac{\Gamma(\underline{k}-1)}{(4 \pi)^{\underline{k}-1}\left|D_{K}\right|^{\frac{1}{2}}} & \sum_{f \in \mathcal{B}_{\underline{k}}^{\sharp}(\mathfrak{c})} \frac{1}{\langle f, f\rangle} \times \\
\times & \left|\sum_{\mathfrak{b} \mid(\mathfrak{q}, \mathfrak{c})^{\infty}} \frac{\gamma_{f g}(\mathfrak{b})}{(N \mathfrak{b})^{\frac{1}{2}}} \sum_{\mathfrak{a}} \frac{\chi_{\mathfrak{q} \mathfrak{c}}(\mathfrak{a})}{(N \mathfrak{a})} \sum_{\mathfrak{m}} \frac{C_{f}(\mathfrak{m}) C_{g}(\mathfrak{m})}{(N \mathfrak{m})^{\frac{1}{2}}} V_{\frac{1}{2}}\left(\frac{(2 \pi)^{2 d} N\left(\mathfrak{b} \mathfrak{a}^{2} \mathfrak{m}\right)}{D_{K}^{2} N\left(\frac{\mathfrak{q} \mathfrak{c}}{(\mathfrak{q}, \mathfrak{c})}\right)}\right)\right|^{2} .
\end{aligned}
$$

We use the estimate 4.1.10 to bound the coefficients $\gamma_{f g}$ to obtain

$$
\begin{aligned}
& M^{\sharp}(g) \ll \\
& 4 \frac{\Gamma(\underline{k}-1)}{(4 \pi)^{\underline{k}-1}\left|D_{K}\right|^{\frac{1}{2}}} \sum_{f \in \mathcal{B}_{\underline{k}}^{\sharp}(\mathfrak{c})} \frac{1}{\langle f, f\rangle}\left(\sum_{\mathfrak{b} \mid(\mathfrak{q}, \mathfrak{c})^{\infty}}|N \mathfrak{b}|^{\varepsilon-\frac{1}{2}}\left|\sum_{\mathfrak{a}} \frac{\chi_{\mathfrak{q} \mathfrak{c}(\mathfrak{a})}}{(N \mathfrak{a})} \sum_{\mathfrak{m}} \frac{C_{f}(\mathfrak{m}) C_{g}(\mathfrak{m})}{(N \mathfrak{m})^{\frac{1}{2}}} V_{\frac{1}{2}}\left(\frac{(2 \pi)^{2 d} N\left(\mathfrak{b} \mathfrak{a}^{2} \mathfrak{m}\right)}{D_{K}^{2} N\left(\frac{\mathfrak{q} \mathfrak{c}}{(\mathfrak{q}, \mathfrak{c})}\right)}\right)\right|\right)^{2} .
\end{aligned}
$$

To apply a spectral summation formula, we need to extend the basis $\mathcal{B}_{\underline{k}}^{\sharp}(\mathfrak{c})$ of primitive newforms to an orthogonal basis $\mathcal{B}_{\underline{k}}(\mathfrak{c})$ of the complete space $S_{\underline{\underline{k}}}(\mathfrak{c})$. This is possible, since the terms are all non-negative. We will denote the right hand side of 4.2 .2 over the extended basis by $M(g)$. Thus,

$$
\begin{aligned}
M^{\sharp}(g) \ll M(g):= & \frac{\Gamma(\underline{k}-1)}{(4 \pi)^{\underline{k}-1}\left|D_{K}\right|^{\frac{1}{2}}} \sum_{f \in \mathcal{B}_{\underline{\underline{k}}}^{\sharp}(\mathfrak{c})} \frac{1}{\langle f, f\rangle} \times \\
& \times\left(\sum_{\mathfrak{b} \mid(\mathfrak{q}, \mathfrak{c})^{\infty}}|N \mathfrak{b}|^{\varepsilon-\frac{1}{2}}\left|\sum_{\mathfrak{a}} \frac{\chi_{\mathfrak{q} \mathfrak{c}}(\mathfrak{a})}{(N \mathfrak{a})} \sum_{\mathfrak{m}} \frac{C_{f}(\mathfrak{m}) C_{g}(\mathfrak{m})}{(N \mathfrak{m})^{\frac{1}{2}}} V_{\frac{1}{2}}\left(\frac{(2 \pi)^{2 d} N\left(\mathfrak{b} \mathfrak{a}^{2} \mathfrak{m}\right)}{D_{K}^{2} N\left(\frac{\mathfrak{q} \mathfrak{c}}{(\mathfrak{q}, \mathfrak{c})}\right)}\right)\right|\right)^{2} .
\end{aligned}
$$

Next, we write out the square to get an expression to which we can apply Petersson's trace formula. Note that the Fourier coefficients $C_{f}(\mathfrak{m})$ and $C_{g}(\mathfrak{m})$ as well as the function $V_{\frac{1}{2}}$ are real-valued and we may assume that the $N \mathfrak{b}$ is non-negative, as every ideal has a totally positive generator. We obtain

$$
\begin{aligned}
M(g)= & 4 \sum_{\substack{\mathfrak{b}_{\iota} \mid(\mathfrak{q}, \mathfrak{c})^{\infty} \\
\iota=1,2}}\left|N\left(\mathfrak{b}_{1} \mathfrak{b}_{2}\right)\right|^{\varepsilon-\frac{1}{2}} \sum_{\mathfrak{a}_{1}, \mathfrak{a}_{2}} \frac{\chi_{\mathfrak{q} \mathfrak{c}}\left(\mathfrak{a}_{1} \mathfrak{a}_{2}\right)}{N\left(\mathfrak{a}_{1} \mathfrak{a}_{2}\right)} \sum_{\mathfrak{m}_{1}, \mathfrak{m}_{2}} \frac{C_{g}\left(\mathfrak{m}_{1}\right) C_{g}\left(\mathfrak{m}_{2}\right)}{\left(N\left(\mathfrak{m}_{1} \mathfrak{m}_{2}\right)\right)^{\frac{1}{2}}} V_{\frac{1}{2}}\left(\frac{(2 \pi)^{2 d} N\left(\mathfrak{b}_{1} \mathfrak{a}_{1}^{2} \mathfrak{m}_{1}\right)}{D_{K}^{2} N\left(\frac{\mathfrak{q} \mathfrak{c}}{(\mathfrak{q}, \mathfrak{c})}\right)}\right) \times \\
& \times V_{\frac{1}{2}}\left(\frac{(2 \pi)^{2 d} N\left(\mathfrak{b}_{2} \mathfrak{a}_{2}^{2} \mathfrak{m}_{2}\right)}{D_{K}^{2} N\left(\frac{\mathfrak{q} \mathfrak{c}}{(\mathfrak{q}, \mathfrak{c})}\right)}\right) \frac{\Gamma(\underline{k}-1)}{(4 \pi)^{\underline{k}-1}\left|D_{K}\right|^{\frac{1}{2}}} \sum_{f \in \mathcal{B}_{\underline{k}}(\mathfrak{c})} \frac{C_{f}\left(\mathfrak{m}_{1}\right) C_{f}\left(\mathfrak{m}_{2}\right)}{\langle f, f\rangle} .
\end{aligned}
$$


Applying Petersson's trace formula, i.e. Lemma 4.1.6, to the innermost sum we get

$$
\begin{aligned}
M(g)= & 4 \sum_{\substack{\mathfrak{b}_{\iota} \mid(\mathfrak{q}, \mathfrak{c}) \\
\iota=1,2}}\left|N\left(\mathfrak{b}_{1} \mathfrak{b}_{2}\right)\right|^{\varepsilon-\frac{1}{2}} \sum_{\mathfrak{a}_{1}, \mathfrak{a}_{2}} \frac{\chi_{\mathfrak{q} \mathfrak{c}}\left(\mathfrak{a}_{1} \mathfrak{a}_{2}\right)}{N\left(\mathfrak{a}_{1} \mathfrak{a}_{2}\right)} \sum_{\substack{\mathfrak{m}_{j}=\left(m_{j}\right) \\
j=1,2}} \frac{C_{g}\left(\mathfrak{m}_{1}\right) C_{g}\left(\mathfrak{m}_{2}\right)}{\left(N\left(\mathfrak{m}_{1} \mathfrak{m}_{2}\right)\right)^{\frac{1}{2}}} \times \\
& \times V_{\frac{1}{2}}\left(\frac{(2 \pi)^{2 d} N\left(\mathfrak{b}_{1} \mathfrak{a}_{1}^{2} \mathfrak{m}_{1}\right)}{D_{K}^{2} N\left(\frac{\mathfrak{q} \mathfrak{c}}{(\mathfrak{q}, \mathfrak{c})}\right)}\right) V_{\frac{1}{2}}\left(\frac{(2 \pi)^{2 d} N\left(\mathfrak{b}_{2} \mathfrak{a}_{2}^{2} \mathfrak{m}_{2}\right)}{D_{K}^{2} N\left(\frac{\mathfrak{q} \mathfrak{c}}{(\mathfrak{q}, \mathfrak{c})}\right)}\right) \times \\
& \times\left[\delta_{m_{1}=\epsilon^{2} m_{2}}+\frac{(2 \pi)^{d}(-1)^{d \frac{k}{2}}}{\left|D_{K}\right|^{\frac{1}{2}}} \sum_{\epsilon \in \mathfrak{o}^{\times}} \sum_{c \in \mathfrak{c}^{*} / \mathfrak{o}^{\times}} \frac{S_{K}\left(m_{1}, \epsilon^{2} m_{2} ; c\right)}{|N c|} \mathbf{J}_{\underline{k}-1}\left(\frac{4 \pi \sqrt{m_{1} m_{2}|\epsilon|}}{|c|}\right)\right] \\
= & M_{\delta}(g)+M_{S}(g) .
\end{aligned}
$$

In the following we estimate $M(g)$.

To begin we consider the diagonal term, i.e.

$$
\begin{aligned}
M_{\delta}(g)= & 4 \sum_{\substack{\mathfrak{b}_{\iota} \mid(\mathfrak{q}, \mathfrak{c})^{\infty} \\
\iota=1,2}}\left(N\left(\mathfrak{b}_{1} \mathfrak{b}_{2}\right)\right)^{\varepsilon-\frac{1}{2}} \sum_{\mathfrak{a}_{1}, \mathfrak{a}_{2}} \frac{\chi_{\mathfrak{q} \mathfrak{c}}\left(\mathfrak{a}_{1} \mathfrak{a}_{2}\right)}{N\left(\mathfrak{a}_{1} \mathfrak{a}_{2}\right)} \times \\
& \times \sum_{\mathfrak{m}=(m)} \frac{C_{g}^{2}(\mathfrak{m})}{N(\mathfrak{m})} V_{\frac{1}{2}}\left(\frac{(2 \pi)^{2 d} N\left(\mathfrak{b}_{1} \mathfrak{a}_{1}^{2} \mathfrak{m}\right)}{D_{K}^{2} N\left(\frac{\mathfrak{q} \mathfrak{c}}{(\mathfrak{q}, \mathfrak{c})}\right)}\right) V_{\frac{1}{2}}\left(\frac{(2 \pi)^{2 d} N\left(\mathfrak{b}_{2} \mathfrak{a}_{2}^{2} \mathfrak{m}\right)}{D_{K}^{2} N\left(\frac{\mathfrak{q} \mathfrak{c}}{(\mathfrak{q}, \mathfrak{c})}\right)}\right) .
\end{aligned}
$$

We use the decay property of $V_{\frac{1}{2}}$ given in 4.1.11 to see that the multiple sum is absolutely convergent and bounded by

$$
\begin{aligned}
M_{\delta}(g) \ll & \sum_{\substack{\mathfrak{b}_{\iota} \mid(\mathfrak{q}, \mathfrak{c})^{\infty} \\
\iota=1,2}}\left(N\left(\mathfrak{b}_{1} \mathfrak{b}_{2}\right)\right)^{\varepsilon-\frac{1}{2}} \sum_{\mathfrak{a}_{1}, \mathfrak{a}_{2}} \frac{\chi_{\mathfrak{q} \mathfrak{c}}\left(\mathfrak{a}_{1} \mathfrak{a}_{2}\right)}{N\left(\mathfrak{a}_{1} \mathfrak{a}_{2}\right)} \times \\
& \times \sum_{\substack{\mathfrak{m}=(m)\\
}} \frac{C_{g}^{2}(\mathfrak{m})}{N(\mathfrak{m})}\left(1+\frac{N\left(\mathfrak{b}_{1} \mathfrak{a}_{1}^{2} \mathfrak{m}\right)}{k^{d} D_{K}^{2} N\left(\frac{\mathfrak{q} \mathfrak{c}}{(\mathfrak{q}, \mathfrak{c})}\right)}\right)^{-A}\left(1+\frac{N\left(\mathfrak{b}_{2} \mathfrak{a}_{2}^{2} \mathfrak{m}\right)}{k^{d} D_{K}^{2} N\left(\frac{\mathfrak{q} \mathfrak{c}}{(\mathfrak{q}, \mathfrak{c})}\right)}\right)^{-A} \\
& \ll\left(k^{d} N \frac{\mathfrak{q} \mathfrak{c}}{(\mathfrak{q}, \mathfrak{c})}\right)^{3 \varepsilon} .
\end{aligned}
$$

Thus we have the intermediate result $M^{\sharp}(g) \leq M(g)=M_{S}(g)+\mathcal{O}\left(k^{3 d \varepsilon}\left(N \frac{\mathfrak{q} \mathfrak{c}}{(\mathfrak{q}, \mathfrak{c})}\right)^{3 \varepsilon}\right)$.

We are left with the off-diagonal term $M_{S}(g)$. We shall split this sum into several pieces, then we bound some of them using trivial estimates. For the remaining terms, where $N\left(\mathfrak{m}_{j}\right)$ and $N(c)$ are relatively small, we find bounds in Section 4.2 .2 .

In the interest of readability, we fix $\mathfrak{a}_{\iota}$ and $\mathfrak{b}_{\iota}, \iota=1,2$ and restrict our attention to the sums over $\mathfrak{m}_{j}$ and $c$.

Let $\left\{\phi_{j}\right\}_{j \in \mathbb{N}_{0}}$ be a family of smooth functions such that

$$
\operatorname{supp} \phi_{j} \subseteq\left[\frac{1}{4}, 4\right], \quad x^{\nu} \frac{d^{\nu}}{d x^{\nu}} \phi_{j}(x) \ll_{\nu} 1, \quad \sum_{j \in \mathbb{N}_{0}} \phi_{j}\left(\frac{x}{2^{j}}\right) \equiv 1, \quad \text { for all } x \in \mathbb{R}_{\geq 1}, \nu \in \mathbb{N}_{0} .
$$

Define $M_{1 i}=2^{i}, M_{2 j}=2^{j}$ and $Q_{l}=2^{l}$. Then, we consider for the triples $(i, j, l)$ the cuboids

$$
\left(N m_{1}, N m_{2}, N c\right) \in\left[\frac{M_{1 i}}{4}, 4 M_{1 i}\right] \times\left[\frac{M_{2 j}}{4}, 4 M_{2 j}\right] \times\left[\frac{Q_{l}}{4}, 4 Q_{l}\right] .
$$


For $(i, j, l) \in \mathbb{N}_{0}^{3}$ we define

$$
\begin{aligned}
M_{i j l}= & \sum_{\epsilon \in \mathfrak{o}^{\times}} \sum_{c \in \mathfrak{c}^{*} / \mathfrak{o}^{\times}}|N c|^{-1} \phi_{l}\left(\frac{|N c|}{2^{l}}\right) \sum_{\substack{\mathfrak{m}_{j}=\left(m_{j}\right) \\
j=1,2}} \phi_{i}\left(\frac{N m_{1}}{2^{i}}\right) \phi_{j}\left(\frac{N m_{2}}{2^{j}}\right) \frac{C_{g}\left(\mathfrak{m}_{1}\right) C_{g}\left(\mathfrak{m}_{2}\right)}{\left(N\left(\mathfrak{m}_{1} \mathfrak{m}_{2}\right)\right)^{\frac{1}{2}}} \times \\
& \times V_{\frac{1}{2}}\left(\frac{(2 \pi)^{2 d} N\left(\mathfrak{b}_{1} \mathfrak{a}_{1}^{2} \mathfrak{m}_{1}\right)}{D_{K}^{2} N\left(\frac{\mathfrak{q} \mathfrak{c}}{(\mathfrak{q}, \mathfrak{c})}\right)}\right) V_{\frac{1}{2}}\left(\frac{(2 \pi)^{2 d} N\left(\mathfrak{b}_{2} \mathfrak{a}_{2}^{2} \mathfrak{m}_{2}\right)}{D_{K}^{2} N\left(\frac{\mathfrak{q} \mathfrak{c}}{(\mathfrak{q}, \mathfrak{c})}\right)}\right) S_{K}\left(m_{1}, \epsilon^{2} m_{2} ; c\right) \mathbf{J}_{\underline{k}-1}\left(\frac{4 \pi \sqrt{m_{1} m_{2}}|\epsilon|}{|c|}\right) .
\end{aligned}
$$

Thus, we have

$$
M_{S}(g)=4 \frac{(2 \pi)^{d}(-1)^{d \frac{k}{2}}}{\left|D_{K}\right|^{\frac{1}{2}}} \sum_{\substack{\mathfrak{b}_{j} \mid(\mathfrak{q}, \mathfrak{c})^{\infty} \\ j=1,2}}\left(N\left(\mathfrak{b}_{1} \mathfrak{b}_{2}\right)\right)^{\varepsilon-\frac{1}{2}} \sum_{\substack{\mathfrak{a}_{j} \\ j=1,2}} \frac{\chi_{\mathfrak{q} \mathfrak{c}}\left(\mathfrak{a}_{1} \mathfrak{a}_{2}\right)}{N\left(\mathfrak{a}_{1} \mathfrak{a}_{2}\right)} \sum_{i, j, l \in \mathbb{N}_{0}} M_{i j l}
$$

We consider the terms $M_{i j l}$ separately. Recall the bounds of the Kloosterman sum $S_{K}$ given in 2.0.3, the bound for the product of Bessel $J$-functions $\mathbf{J}_{\underline{k}-1}$ given in 4.1.18 and the bound 4.1.11 for $V_{\frac{1}{2}}$. Inserting these estimates, we obtain

$$
\begin{aligned}
M_{i j l} \ll & \sum_{\epsilon \in \mathfrak{o}^{\times}} \sum_{c \in \mathfrak{c}^{*} / \mathfrak{o}^{\times}} \sum_{\substack{\mathfrak{m}_{j}=\left(m_{j}\right) \\
j=1,2}} \phi_{i}\left(\frac{N m_{1}}{2^{i}}\right) \phi_{j}\left(\frac{N m_{2}}{2^{j}}\right) \phi_{l}\left(\frac{|N c|}{2^{l}}\right) \frac{\left(N m_{1}, N m_{2}, N c\right)^{\frac{1}{2}}}{N\left(m_{1} m_{2}|c|\right)^{\frac{1}{2}-\varepsilon}} \times \\
& \times\left(1+\frac{N\left(\mathfrak{b}_{1} \mathfrak{a}_{1}^{2} \mathfrak{m}_{1}\right)}{k^{d} D_{K}^{2} N\left(\frac{\mathfrak{q} \mathfrak{c}}{(\mathfrak{q}, \mathfrak{c})}\right)}\right)^{-A}\left(1+\frac{N\left(\mathfrak{b}_{2} \mathfrak{a}_{2}^{2} \mathfrak{m}_{2}\right)}{k^{d} D_{K}^{2} N\left(\frac{\mathfrak{q} \mathfrak{c}}{(\mathfrak{q}, \mathfrak{c})}\right)}\right)^{-A} \min \left\{1,\left(\frac{(16 e \pi)^{d} \sqrt{N m_{1} m_{2}}}{k^{d}|N c|}\right)^{k-1}\right\} \times \\
& \times|N c|^{\eta} \prod_{\left|\epsilon_{j}\right|>1}\left|\epsilon_{j}\right|^{-\eta} \cdot
\end{aligned}
$$

Note that $0<\eta<\frac{1}{2}$. The sum over the units is of constant size, i.e.

$$
\sum_{\epsilon \in \mathfrak{o}^{\times}} \prod_{\left|\epsilon_{j}\right|>1}\left|\epsilon_{j}\right|^{-\eta} \ll 1
$$

see [L03, page 136].

Inserting this bound and using 4.2.5 coming from the support of the $\phi$ 's, we get the estimate

$$
\begin{aligned}
M_{i j l} \ll & \left(M_{1 i} M_{2 j}\right)^{\frac{1}{2}+\varepsilon} \frac{Q_{l}^{\frac{1}{2}+2 \varepsilon}}{N \mathfrak{c}}\left(1+\frac{M_{1 i} N\left(\mathfrak{b}_{1} \mathfrak{a}_{1}^{2}\right)}{k^{d} D_{K}^{2} N\left(\frac{\mathfrak{q} \mathfrak{c}}{(\mathfrak{q}, \mathfrak{c})}\right)}\right)^{-A}\left(1+\frac{M_{2 j} N\left(\mathfrak{b}_{2} \mathfrak{a}_{2}^{2}\right)}{k^{d} D_{K}^{2} N\left(\frac{\mathfrak{q} \mathfrak{c}}{(\mathfrak{q}, \mathfrak{c})}\right)}\right)^{-A} \times \\
& \times \min \left\{1,\left(\frac{(16 e \pi)^{d} \sqrt{M_{1 i} M_{2 j}}}{k^{d} Q_{l}}\right)^{k-1}\right\} Q_{l}^{\eta} .
\end{aligned}
$$

Now, we consider separately the three cases when at least one of $Q_{l}, M_{1 i}$ and $M_{2 j}$ is relatively large. First let

$$
Q_{l} \geq \frac{(64 e \pi)^{d} \sqrt{M_{1 i} M_{2 j}}}{k^{d}}
$$

Thus

$$
\min \left\{1,\left(\frac{(16 e \pi)^{d} \sqrt{M_{1 i} M_{2 j}}}{k^{d} Q_{l}}\right)^{k-1}\right\} Q_{l}^{\eta} \leq\left(\frac{(16 e \pi)^{d} \sqrt{M_{1 i} M_{2 j}}}{k^{d} Q_{l}}\right)^{k-1} Q_{l}^{\eta},
$$


and the sum over $l$ can be estimated by

$$
\begin{aligned}
& \left((16 e \pi)^{d} \sqrt{M_{1 i} M_{2 j}}\right)^{k-1} k^{-d(k-1)} \sum_{Q_{l} \geq \frac{(64 e \pi)^{d} \sqrt{M_{1 i} M_{2 j}}}{k^{d}}} Q_{l}^{-k+\frac{3}{2}+2 \varepsilon+\eta} \\
\ll & 4^{-k d} k^{-\frac{3}{2} d}\left(M_{1 i} M_{2 j}\right)^{\frac{3}{4}+\varepsilon+\frac{\eta}{2}} .
\end{aligned}
$$

Using standard estimates for the sum over $i, j$ we obtain the bound

$$
\begin{aligned}
& \sum_{i, j \in \mathbb{N}_{0}}\left(M_{1 i} M_{2 j}\right)^{\frac{5}{4}+2 \varepsilon+\frac{\eta}{2}}\left(1+\frac{M_{1 i} N\left(\mathfrak{b}_{1} \mathfrak{a}_{1}^{2}\right)}{k^{d} D_{K}^{2} N\left(\frac{\mathfrak{q} \mathfrak{c}}{(\mathfrak{q}, \mathfrak{c})}\right)}\right)^{-A}\left(1+\frac{M_{2 j} N\left(\mathfrak{b}_{2} \mathfrak{a}_{2}^{2}\right)}{k^{d} D_{K}^{2} N\left(\frac{\mathfrak{q} \mathfrak{c}}{(\mathfrak{q}, \mathfrak{c})}\right)}\right)^{-A} \\
\ll & k^{\frac{9}{2} d+\varepsilon(4 d+5)+d \eta}\left(N \frac{\mathfrak{q} \mathfrak{c}}{(\mathfrak{q}, \mathfrak{c})}\right)^{\frac{9}{2}+2 \varepsilon+\eta}\left(N\left(\mathfrak{b}_{1} \mathfrak{b}_{2} \mathfrak{a}_{1}^{2} \mathfrak{a}_{2}^{2}\right)\right)^{-\frac{9}{4}}
\end{aligned}
$$

Combining the estimates, we obtain

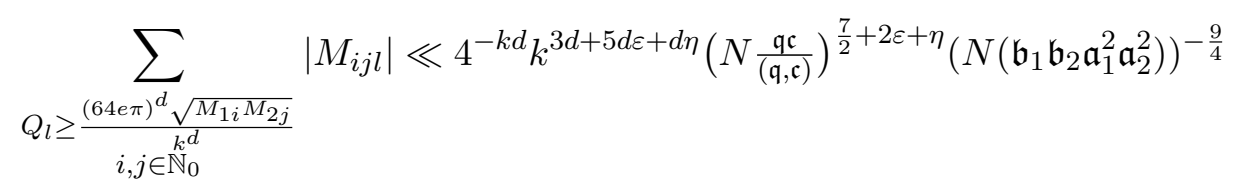

which is negligible as $k$ is large relatively to $N \mathfrak{q}$.

Now we consider the case that at least one of $M_{1 i}$ and $M_{2 j}$ is large. The expression is symmetric in $m_{1}$ and $m_{2}$. Therefore it is enough to assume $M_{1 i}$ to be large, more precisely

$$
M_{1 i} \geq \frac{k^{d+\varepsilon} D_{K}^{2} N\left(\frac{\mathfrak{q} \mathfrak{c}}{(\mathfrak{q}, \mathfrak{c})}\right)}{N\left(\mathfrak{b}_{1} \mathfrak{a}_{1}^{2}\right)}
$$

such that $\left(1+\frac{M_{1 i} N\left(\mathfrak{b}_{1} \mathfrak{a}_{1}^{2}\right)}{k^{d} D_{K}^{2} N\left(\frac{\mathfrak{q} \mathfrak{c}}{(\mathfrak{q}, \mathfrak{c})}\right)}\right)^{-A} \leq\left(\frac{M_{1 i} N\left(\mathfrak{b}_{1} \mathfrak{a}_{1}^{2}\right)}{k^{d} D_{K}^{2} N\left(\frac{\mathfrak{q} \mathfrak{c}}{(\mathfrak{q}, \mathfrak{c})}\right)}\right)^{-A}$ hold.

Summing over $l$, we obtain

$$
\sum_{l \in \mathbb{N}_{0}} Q_{l}^{\frac{1}{2}+2 \varepsilon+\eta} \min \left\{1,\left(\frac{(16 e \pi)^{d} \sqrt{M_{1 i} M_{2 j}}}{k^{d} Q_{l}}\right)^{k-1}\right\} \ll\left(M_{1 i} M_{2 j}\right)^{\frac{3}{4}+\varepsilon+\frac{\eta}{2}} k^{-d(2+2 \varepsilon)} .
$$

Now, we sum over $i, j$, such that 4.2.7 holds. We get

$$
\begin{aligned}
& \sum_{M_{1 i} \geq \frac{k^{d+\varepsilon_{D_{K}}^{2} N\left(\frac{\mathfrak{q} \mathfrak{c}}{(\mathfrak{q}, \mathfrak{c})}\right)}}{N\left(\mathfrak{b}_{1} \mathfrak{a}_{1}^{2}\right)}}\left(M_{1 i} M_{2 j}\right)^{\frac{5}{4}+2 \varepsilon+\frac{\eta}{2}}\left(\frac{M_{1 i} N\left(\mathfrak{b}_{1} \mathfrak{a}_{1}^{2}\right)}{k^{d} D_{K}^{2} N\left(\frac{\mathfrak{q} \mathfrak{c}}{(\mathfrak{q}, \mathfrak{c})}\right)}\right)^{-A}\left(1+\frac{M_{2 j} N\left(\mathfrak{b}_{2} \mathfrak{a}_{2}^{2}\right)}{k^{d} D_{K}^{2} N\left(\frac{\mathfrak{q} \mathfrak{c}}{(\mathfrak{q}, \mathfrak{c})}\right)}\right)^{-A} \\
& \ll k^{-A \varepsilon+d(5+7 \varepsilon)}\left(N \frac{\mathfrak{q} \mathfrak{c}}{(\mathfrak{q}, \mathfrak{c})}\right)^{5+4 \varepsilon}\left(N\left(\mathfrak{b}_{1} \mathfrak{b}_{2} \mathfrak{a}_{1}^{2} \mathfrak{a}_{2}^{2}\right)\right)^{-\frac{9}{4}} .
\end{aligned}
$$

Thus we obtain

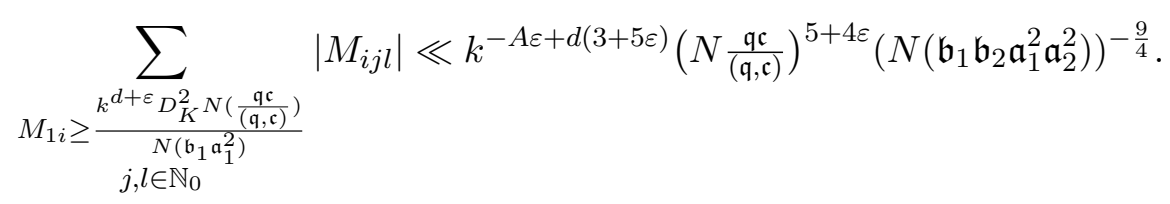


The sums over $\mathfrak{a}_{\iota}$ and $\mathfrak{b}_{\iota}$ are bounded:

$$
\sum_{\substack{\mathfrak{a}_{\iota} \\ \iota=1,2}} \frac{\chi_{\mathfrak{q} \mathfrak{c}}\left(\mathfrak{a}_{1} \mathfrak{a}_{2}\right)}{N\left(\mathfrak{a}_{1} \mathfrak{a}_{2}\right)^{5}} \ll 1, \quad \sum_{\substack{N \mathfrak{b}_{\iota} \mid(\mathfrak{q}, \mathfrak{c})^{\infty} \\ \iota=1,2}}\left(N\left(\mathfrak{b}_{1} \mathfrak{b}_{2}\right)\right)^{\varepsilon-2} \ll 1 .
$$

Choosing $A=\frac{100 d}{\varepsilon^{2}}$, we obtain the intermediate result

$$
\begin{aligned}
& M_{S}(g)=\mathcal{O}\left(k^{-97 d / \varepsilon}\left(N \frac{\mathfrak{q} \mathfrak{c}}{(\mathfrak{q}, \mathfrak{c})}\right)^{5+\varepsilon}\right) \\
& +4 \frac{(2 \pi)^{d}(-1)^{d \frac{k}{2}}}{\left|D_{K}\right|^{\frac{1}{2}}} \sum_{\substack{\mathfrak{b}_{\iota} \mid(\mathfrak{q}, \mathfrak{c})^{\infty} \\
\mathfrak{b}_{\iota} \leq \sqrt{k^{d+\varepsilon} D_{K}^{2} N\left(\frac{\mathfrak{q} \mathfrak{c}}{(\mathfrak{q}, \mathfrak{c})}\right)}}}\left(N\left(\mathfrak{b}_{1} \mathfrak{b}_{2}\right)\right)^{\varepsilon-\frac{1}{2}} \sum_{\substack{N \mathfrak{a}_{\iota} \leq \sqrt{k^{d+\varepsilon} D_{K}^{2} N\left(\frac{\mathfrak{q} \mathfrak{c}}{(\mathfrak{q}, \mathfrak{c})}\right.} \\
\iota=1,2}} \frac{\chi_{\mathfrak{q} \mathfrak{c}}\left(\mathfrak{a}_{1} \mathfrak{a}_{2}\right)}{N\left(\mathfrak{a}_{1} \mathfrak{a}_{2}\right)} \sum_{\substack{(i, j, l) \in C\\
}} M_{i j l}
\end{aligned}
$$

where

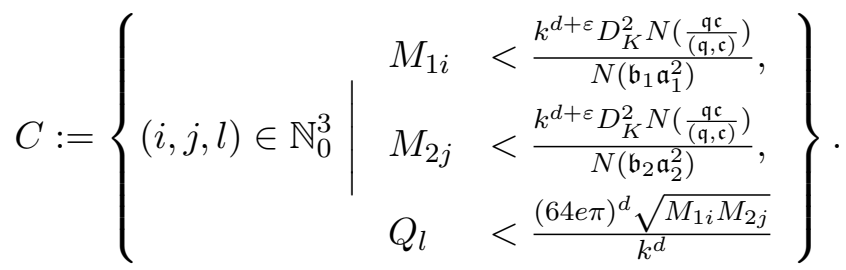

As $k$ is large the error term is negligible. The triples $(i, j, l) \in C$ will be considered in the Section 4.2 .2 .

\subsubsection{Application of Voronoi's Summation Formula}

To estimate the remaining $M_{i j l}$ with $(i, j, l) \in C$, we apply the Voronoi summation formula stated in Theorem 4.1.5. Fix a triple $(i, j, l) \in C$.

We modify the sum over $\mathfrak{m}_{1}$ in 4.2 .6 by Theorem 4.1 .5 and obtain

$$
\begin{aligned}
& \sum_{\substack{\mathfrak{m}_{1}=\left(m_{1}\right) \\
m_{1} \in \mathcal{F}}} C_{g}\left(\mathfrak{m}_{1}\right) e\left[\frac{a m_{1}}{c}\right] F\left(m_{1}\right) \\
= & \frac{1}{N c} \frac{\eta_{g}\left(\frac{q}{(c, q)}\right)}{N\left(\frac{q}{(c, q)}\right)^{\frac{1}{2}}} \sum_{\substack{\mathfrak{n}=(n) \\
n \in \mathcal{F}}} C_{g}(\mathfrak{n}) e\left[-n \frac{\left(a \frac{q}{(c, q)}\right)^{*}}{c}\right] \int_{\substack{(0, \infty)^{d} \\
\int^{d}}} F(\underline{x})\left(2 \pi i^{k}\right)^{d} \mathbf{J}_{\underline{k}-1}\left(\frac{4 \pi \sqrt{n \underline{x}}}{c\left(\frac{q}{(c, q)}\right)^{\frac{1}{2}}}\right) d \underline{x}
\end{aligned}
$$

where

$$
F(\underline{x})=(N \underline{x})^{-\frac{1}{2}} \phi_{i}\left(\frac{N \underline{x}}{2^{i}}\right) V_{\frac{1}{2}}\left(\frac{N\left(\mathfrak{b}_{1} \mathfrak{a}_{1}^{2} \underline{x}\right)}{D_{K}^{2} N\left(\frac{\mathfrak{q} \mathfrak{c}}{(\mathfrak{q}, \mathfrak{c})}\right)}\right) \mathbf{J}_{\underline{k}-1}\left(\frac{4 \pi \sqrt{\underline{x} m_{2}}|\epsilon|}{|c|}\right) .
$$

As $\phi_{i}$ is compactly supported, $F$ has compact support, in particular, $F$ satisfies the conditions of Theorem 4.1.5. Recall that $\left(\frac{q}{(c, q)}\right)^{*}$ is the multiplicative inverse of $\frac{q}{(c, q)}$ modulo $c$.

The Kloosterman sum $S_{K}\left(m_{1}, \epsilon^{2} m_{2} ; c\right)$ in the definition of $M_{i j l}$ in (4.2.6 changes into a Ramanujan sum given by

$$
\sum_{\substack{a \bmod c \\(a, c)=1}} e\left[a\left(\frac{\epsilon^{2} m_{2}-n\left(\frac{q}{(c, q)}\right)^{*}}{c}\right)\right]=: r_{c}\left(\epsilon^{2} m_{2}-n\left(\frac{q}{(c, q)}\right)^{*}\right) .
$$


Thus, the multiple sum $M_{i j l}$ changes into

$$
\begin{aligned}
M_{i j l}= & \sum_{\epsilon \in \mathfrak{o}^{\times}} \sum_{c \in \mathfrak{c}^{*} / \mathfrak{o}^{\times}} \phi_{l}\left(\frac{|N c|}{2^{l}}\right) \frac{1}{N c^{2}} \frac{\eta_{g}\left(\frac{q}{(c, q)}\right)}{N\left(\frac{q}{(c, q)}\right)^{\frac{1}{2}}} \sum_{\mathfrak{m}_{2}=\left(m_{2}\right)} \phi_{j}\left(\frac{N m_{2}}{2^{j}}\right) \frac{C_{g}\left(\mathfrak{m}_{2}\right)}{\left(N m_{2}\right)^{\frac{1}{2}}} V_{\frac{1}{2}}\left(\frac{N\left(\mathfrak{b}_{2} \mathfrak{a}_{2}^{2} \mathfrak{m}_{2}\right)}{D_{K}^{2} N\left(\frac{\mathfrak{q} \mathfrak{c}}{(\mathfrak{q}, \mathfrak{c})}\right)}\right) \times \\
& \times \sum_{\mathfrak{n}=(n)} C_{g}(\mathfrak{n}) r_{c}\left(\epsilon^{2} m_{2}-n\left(\frac{q}{(c, q)}\right)^{*}\right) \int \ldots \int_{(0, \infty)^{d}} F(\underline{x})\left(2 \pi i^{k}\right)^{d} \mathbf{J}_{\underline{k}-1}\left(\frac{4 \pi \sqrt{n \underline{x}}}{c\left(\frac{q}{(c, q)}\right)^{\frac{1}{2}}}\right) d \underline{x} .
\end{aligned}
$$

To estimate the multiple sum $M_{i j l}$ we divide the sums over $n$ into two parts depending on the size of $n$, namely into $N n \geq\left(N m_{2}\right)^{1+\varepsilon}$ and $N n<\left(N m_{2}\right)^{1+\varepsilon}$. We denote their contributions by $M_{i j l}^{>}$ respectively $M_{i j l}^{<}$, and hence $M_{i j l}=M_{i j l}^{>}+M_{i j l}^{<}$.

\section{Case I $N n \geq\left(N m_{2}\right)^{1+\varepsilon}$}

We denote the partial sum of the second line of 4.2.11) extended over $N n \geq\left(N m_{2}\right)^{1+\varepsilon}$ by $R_{i j l}$.

To estimate $R_{i j l}$, we modify the $\underline{x}$-integral via the substitution $x \mapsto x^{2}$. Let $\alpha \in \mathbb{N}$. Then, we use integration by parts $\alpha$-times in each variable. Therefore we add in the $j$-th step the factor $(N \underline{x})^{-1}(N \underline{x})^{-\underline{k}+1-\underline{j}}(N \underline{x})^{\underline{k}+\underline{j}}$ and use [GR07, 5.52.1] to integrate $(N \underline{x})^{\underline{k}+\underline{j}} \mathbf{J}_{\underline{k}-1+\underline{j}}(\sqrt{n} \underline{x})$ and GR07. 5.52.2] to derive the factor $(N \underline{x})^{-\underline{k}+1-\underline{j}} \underline{\mathbf{J}}_{\underline{k}-1+\underline{j}}\left(\sqrt{m_{2}} \underline{x}\right)$ of $F\left(\underline{x}^{2}\right)$. We use the product rule to derive $F\left(\underline{x}^{2}\right)$ and obtain a sum. By the properties of $\phi_{i}$ and $V_{\frac{1}{2}}$ as well as the estimate 4.1.18) for the Bessel $J$-functions we obtain that the whole expression is bounded uniformly in $\underline{k}$ by:

$$
\begin{aligned}
& \int_{(0, \infty)^{d}} \ldots \int_{\mid} F(\underline{x})\left(2 \pi i^{k}\right)^{d} \mathbf{J}_{\underline{k}-1}\left(\frac{4 \pi \sqrt{n \underline{x}}}{c\left(\frac{q}{(c, q)}\right)^{\frac{1}{2}}}\right) d \underline{x} \\
& \ll|N c|^{2 \eta} \prod_{\left|\epsilon_{j}\right|>1}\left|\epsilon_{j}\right|^{-\eta}\left(\frac{1}{N n}\right)^{\frac{\alpha}{2}} \int_{(0, \infty)^{d}} \ldots \int_{i} \phi_{i}\left(\frac{N \underline{x}^{2}}{2^{i}}\right) V_{\frac{1}{2}}\left(\frac{N\left(\mathfrak{b}_{1} \mathfrak{a}_{1}^{2} \underline{x}^{2}\right)}{D_{K}^{2} N\left(\frac{\underline{\mathfrak{q}}}{(\mathfrak{q}, \mathfrak{c})}\right)}\right) \sum_{0 \leq \beta \leq \alpha}\left(N m_{2}\right)^{\frac{\beta}{2}}(N \underline{x})^{\alpha-\beta} d \underline{x} \\
& \ll \prod_{\left|\epsilon_{j}\right|>1}\left|\epsilon_{j}\right|^{-\eta}|N c|^{2 \eta} \sqrt{M_{1 i}}\left(\frac{1}{N n}\right)^{\frac{\alpha}{2}} \sum_{0 \leq \beta \leq \alpha}\left(N m_{2}\right)^{\frac{\beta}{2}}{\sqrt{M_{1 i}}}^{\alpha-\beta} .
\end{aligned}
$$

The second bound follows by the compact support of $\phi_{i}$. Now we sum over $n$, to get

$$
\begin{aligned}
R_{i j l} & \ll \prod_{\left|\epsilon_{j}\right|>1}\left|\epsilon_{j}\right|^{-\eta}|N c|^{2 \eta} \sqrt{M_{1 i}} \sum_{N n>\left(N m_{2}\right)^{1+\varepsilon}} C_{g}(\mathfrak{n}) e\left[-n \frac{\left(a \frac{q}{(c, q)}\right)^{*}}{c}\right]\left(\frac{1}{N n}\right)^{\frac{\alpha}{2}} \sum_{0 \leq \beta \leq \alpha}\left(N m_{2}\right)^{\frac{\beta}{2}}{\sqrt{M_{1 i}}}^{\alpha-\beta} \\
& \ll \prod_{\left|\epsilon_{j}\right|>1}\left|\epsilon_{j}\right|^{-\eta}|N c|^{2 \eta} \sqrt{M_{1 i}}\left(N m_{2}\right)^{-\frac{\alpha}{2}-\frac{\alpha}{2} \varepsilon+2 \varepsilon+1} \sum_{0 \leq \beta \leq \alpha}\left(N m_{2}\right)^{\frac{\beta}{2}}{\sqrt{M_{1 i}}}^{\alpha-\beta} .
\end{aligned}
$$

Inserting this estimate into 4.2.11) and summing over $\epsilon, c$ and $m_{2}$ we obtain that $M_{i j l}^{>}$is bounded by

$$
M_{i j l}^{\gtrless} \ll Q_{l}^{-1+2 \eta} M_{1 i}^{\frac{1}{2}} M_{2 j}^{\frac{3}{2}-\frac{\alpha}{2}-\varepsilon \frac{\alpha}{2}+3 \varepsilon} \sum_{0 \leq \beta \leq \alpha}{\sqrt{M_{2 j}}}^{\beta}{\sqrt{M_{1 i}}}^{\alpha-\beta} .
$$

Now we sum over $i, j$ and $l$, using the bounds 4.2 .9 , i.e. $M_{*} \ll(N \mathfrak{c}) k^{d+\varepsilon},(N \mathfrak{c}) \leq Q_{l} \ll(N \mathfrak{c}) k^{d \varepsilon}$ and $M_{2 j} \geq \frac{k^{2 d} Q_{l}^{2}}{(64 e \pi)^{d} M_{1 i}}$. We get

$$
\sum_{(i, j, l) \in C}\left|M_{i j l}^{\geq}\right| \ll k^{d\left(2-\frac{\alpha}{2} \varepsilon+8 \varepsilon\right)}\left(N \frac{\mathfrak{q} \mathfrak{c}}{(\mathfrak{q}, \mathfrak{c})}\right)^{2-\frac{\alpha}{2} \varepsilon+6 \varepsilon} .
$$


Finally we choose $\alpha=\left\lceil\frac{6}{\varepsilon}\right\rceil$ to see that this part is negligible.

Case II $N n<\left(N m_{2}\right)^{1+\varepsilon}$

To get a bound for the sum containing small $n$, we have to estimate the integral more carefully. We apply 4.1.17 to reformulate the product of the two Bessel $J$-functions. Let

$$
\mathcal{V}(\underline{x})=(N \underline{x})^{-\frac{1}{2}} \phi_{i}\left(\frac{N \underline{x}}{2^{i}}\right) V_{\frac{1}{2}}\left(\frac{N\left(\mathfrak{b}_{1} \mathfrak{a}_{1}^{2} \underline{x}\right)}{D_{K}^{2} N\left(\frac{\mathfrak{q} \mathfrak{c}}{(\mathfrak{q}, \mathfrak{c})}\right)}\right) .
$$

Then the integral in 4.2.10 changes to

$$
2^{d} \operatorname{Re} \int_{(0, \infty)^{d}} \ldots \iint_{(0, \infty)^{d}} \ldots \int_{0} e\left[\left(n \frac{(c, q)}{q}+|\epsilon|^{2} m_{2}\right) \underline{\gamma}+\frac{\underline{x}}{c^{2} \underline{\gamma}}\right] \mathbf{J}_{\underline{k}-1}\left(4 \pi \sqrt{n m_{2}}|\epsilon|\left(\frac{(c, q)}{q}\right)^{\frac{1}{2}} \underline{\gamma}\right) \mathcal{V}(\underline{x}) d \underline{x} \frac{d \underline{\gamma}}{N \underline{\gamma}}
$$

By the compact support of $\mathcal{V}$ and the rapid decay of $\mathbf{J}_{\underline{k}-1}$ this multi-integral is absolutely convergent. Extending the region of integration of the integral over $\underline{x}$ to the region $(-\infty, \infty)^{d}$ we see that this is the Fourier transform of $\mathcal{V}$ :

$$
\int_{(-\infty, \infty)^{d}} \cdots \int_{\mathcal{V}} \mathcal{V}(\underline{x}) e\left[\frac{\underline{x}}{c^{2} \underline{\gamma}}\right] d \underline{x}=\widehat{\mathcal{V}}\left(\frac{1}{c^{2} \underline{\gamma}}\right)
$$

As $\mathcal{V}$ is compactly supported in $\left[\frac{M_{1 i}}{4}, 4 M_{1 i}\right]$, we obtain the trivial bound

$$
\left|\widehat{\mathcal{V}}\left(\frac{1}{c^{2} \underline{\gamma}}\right)\right| \ll \sqrt{M_{1 i}}
$$

We have to consider the integral

$$
2^{d} \int_{(0, \infty)^{d}} \ldots \int \hat{\mathcal{V}}\left(\frac{1}{c^{2} \underline{\gamma}}\right) e\left[\left(n \frac{(c, q)}{q}+|\epsilon|^{2} m_{2}\right) \underline{\gamma}\right] \mathbf{J}_{\underline{k}-1}\left(4 \pi \sqrt{n m_{2}}|\epsilon|\left(\frac{(c, q)}{q}\right)^{\frac{1}{2}} \underline{\gamma}\right) \frac{d \underline{\gamma}}{N \underline{\gamma}} .
$$

Again we divide the integral. We introduce a smooth partition of unity given by the three smooth functions $\left\{\Psi_{l}, \Psi, \Psi_{u}\right\}$ satisfying the following properties:

$$
\operatorname{supp} \Psi_{l} \subseteq\left[0, M_{1 i}^{1-\varepsilon}(N c)^{-2}\right], \quad \operatorname{supp} \Psi \subseteq\left[\frac{1}{4} M_{1 i}^{1-\varepsilon}(N c)^{-2},(N \mathfrak{c})^{2} k^{4 d}\right], \quad \operatorname{supp} \Psi_{u} \subseteq\left[\frac{1}{4}(N \mathfrak{c})^{2} k^{4 d}, \infty\right)
$$

and

$$
y^{\nu} \frac{d^{\nu}}{d y^{\nu}} \Psi_{*} \ll 1 \text { for } \nu \in \mathbb{N}_{0}, \quad \Psi_{l}(y)+\Psi(y)+\Psi_{u}(y)=1 \text { for all } y \in \mathbb{R}^{+} .
$$

First we estimate the integral over $\Psi_{l}$. We consider $\widehat{\mathcal{V}}$ and note that $N \gamma \leq M_{1 i}^{1-\varepsilon}(N c)^{-2 d}$, then we use integration by parts $\beta$-times to obtain

$$
\widehat{\mathcal{V}}\left(\frac{1}{c^{2} \underline{\gamma}}\right) \ll M_{1 i}^{-\beta+\frac{1}{2}}\left(N c^{2} \underline{\gamma}\right)^{\beta} .
$$


Thus, we get

$$
\begin{aligned}
& 2^{d} \int_{(0, \infty)^{d}} \ldots \int_{l} \Psi_{l}(N \underline{\gamma}) \widehat{\mathcal{V}}\left(\frac{1}{c^{2} \underline{\gamma}}\right) e\left[\left(n \frac{(c, q)}{q}+|\epsilon|^{2} m_{2}\right) \underline{\gamma}\right] \mathbf{J}_{\underline{k}-1}\left(4 \pi \sqrt{n m_{2}}|\epsilon|\left(\frac{(c, q)}{q}\right)^{\frac{1}{2}} \underline{\gamma}\right) \frac{d \underline{\gamma}}{N \underline{\gamma}} \\
\ll & \prod_{\left|\epsilon_{j}\right|>1}\left|\epsilon_{j}\right|^{-\eta} M_{1 i}^{-\beta \varepsilon+\frac{1}{2}-\eta} .
\end{aligned}
$$

For the integral over $\Psi_{u}$ we estimate the Bessel $J$-function by [GR07, 8.451.1] and use the trivial estimate for $\widehat{v}$ given in 4.2.14). We obtain

$$
\begin{aligned}
& 2^{d} \int_{(0, \infty)} \ldots \int_{u} \Psi_{u}(N \underline{\gamma}) \widehat{\mathcal{V}}\left(\frac{1}{c^{2} \underline{\gamma}}\right) e\left[\left(n \frac{(c, q)}{q}+|\epsilon|^{2} m_{2}\right) \underline{\gamma}\right] \mathbf{J}_{\underline{k}-1}\left(4 \pi \sqrt{n m_{2}}|\epsilon|\left(\frac{(c, q)}{q}\right)^{\frac{1}{2}} \underline{\gamma}\right) \frac{d \underline{\gamma}}{N \underline{\gamma}} \\
\ll & \prod_{\left|\epsilon_{j}\right|>1}\left|\epsilon_{j}\right|^{-\eta} M_{1 i}^{\frac{1}{2}}\left(N\left(n m_{2}\right)\right)^{-\frac{1}{4}}(N \mathfrak{c})^{-1} k^{-2 d} .
\end{aligned}
$$

We now use the estimate 4.1.3 and trivially estimate the Ramanujan sum by $\varphi(c)$. We sum over $n$, $m_{2}$ and $c$ to obtain that $M_{i j l}^{<}$with the integral restricted to the support of $\Psi_{u}, \Psi_{l}$ is bounded by

$$
\begin{aligned}
& \sum_{\epsilon \in \mathfrak{o}^{\times}} \prod_{\left|\epsilon_{j}\right|>1}\left|\epsilon_{j}\right|^{-\eta} \sum_{\substack{N n<\left(N m_{2}\right)^{1+\varepsilon} \\
m_{2} \\
c \in \mathfrak{c}^{*} / \mathfrak{o}^{\times}}} \phi_{j}\left(\frac{N m_{2}}{2^{j}}\right) \phi_{l}\left(\frac{|N c|}{2^{l}}\right)\left(N m_{2}\right)^{-\frac{1}{2}+\varepsilon} \frac{\varphi(c)}{(N c)^{-2}} \frac{\eta_{g}\left(\frac{q}{(c, q)}\right)}{N\left(\frac{q}{(c, q)}\right)^{\frac{1}{2}}} \times \\
& \times\left[M_{1 i}^{-\beta \varepsilon+\frac{1}{2}}+M_{1 i}^{\frac{1}{2}}\left(N\left(n m_{2}\right)\right)^{-\frac{1}{4}}(N \mathfrak{c})^{-1} k^{-2 d}\right] \\
& \ll \frac{1}{N \mathfrak{c}}\left[M_{1 i}^{-\beta \varepsilon+\frac{1}{2}} M_{2 j}^{\frac{3}{2}+\varepsilon}+(N \mathfrak{c})^{-1} k^{-2 d} M_{1 i}^{\frac{1}{2}} M_{2 j}^{\frac{5}{4}+2 \varepsilon}\right] .
\end{aligned}
$$

Then, summing over $(i, j, l) \in C$ by using the same estimates as for 4.2 .12 and choosing $\beta=\left\lceil\frac{3}{\varepsilon}\right\rceil$ we see that this part is bounded by

$$
\mathcal{O}\left((N \mathfrak{c})^{-\frac{1}{4}} k^{-\frac{d}{4}+\varepsilon}\right)
$$

We are left with $M_{i j l}^{<}$where the integral 4.2.15) is restricted to the support of $\Psi$. We rewrite the Bessel $J$-function with GR07, 8.411.1] and define $\mathcal{V}(\underline{\gamma})=\widehat{\mathcal{V}}\left(\frac{1}{c^{2} \underline{\gamma}}\right) \frac{1}{N \underline{\gamma}} \Psi(N \underline{\gamma})$. Thus the remaining part is given by

$$
\begin{aligned}
M_{i j l}^{\Psi}:= & \sum_{c \in \mathfrak{c}^{*} / \mathfrak{o}^{\times}} \phi_{l}\left(\frac{|N c|}{2^{l}}\right)|N c|^{-2} \frac{\eta_{g}\left(\frac{q}{(c, q)}\right)}{N\left(\frac{q}{(c, q)}\right)^{\frac{1}{2}}} \int_{(-\pi, \pi)^{d}} \ldots \int e^{-i(k-1) \operatorname{Tr}(\underline{\theta})} \times \\
& \times \sum_{\epsilon \in \mathfrak{o}^{\times}} \sum_{\substack{N n<\left(N m_{2}\right)^{1+\varepsilon} \\
\mathfrak{n}=(n), \mathfrak{m}_{2}=\left(m_{2}\right)}} \phi_{j}\left(\frac{N m_{2}}{2^{j}}\right) r_{c}\left(\epsilon^{2} m_{2}-n\left(\frac{q}{(c, q)}\right)^{*}\right) \frac{C_{g}(\mathfrak{n}) C_{g}\left(\mathfrak{m}_{2}\right)}{\left(N m_{2}\right)^{\frac{1}{2}}} V_{\frac{1}{2}}\left(\frac{N\left(\mathfrak{b}_{2} \mathfrak{a}_{2}^{2} \mathfrak{m}_{2}\right)}{D_{K}^{2} N\left(\frac{\mathfrak{q} \mathfrak{c}}{(\mathfrak{q}, \mathfrak{c})}\right)}\right) \times \\
& \times \frac{1}{\pi^{d}} \int_{(0, \infty)^{d}} \ldots \int_{\substack{d \\
(n)}} e\left[\left(n \frac{(c, q)}{q}+|\epsilon|^{2} m_{2}+2 \sqrt{n m_{2}}|\epsilon|\left(\frac{(c, q)}{q}\right)^{\frac{1}{2}} \sin \underline{\theta}\right) \underline{\gamma}\right] \mathcal{V}(\underline{\gamma}) d \underline{\gamma} d \underline{\theta} .
\end{aligned}
$$

To estimate the integral over $\underline{\gamma}$, we divide the $\left(n, m_{2}, \underline{\theta}\right)$-hypercube into two parts, according as to $N\left(n \frac{(c, q)}{q}+|\epsilon|^{2} m_{2}+2 \sqrt{n m_{2}}|\epsilon|\left(\frac{(c, q)}{q}\right)^{\frac{1}{2}} \sin \underline{\theta}\right)>N\left(\frac{\mathfrak{q} \mathfrak{c}}{(\mathfrak{q}, \mathfrak{c})}\right) k^{d b}$ or $\leq N\left(\frac{\mathfrak{q} \mathfrak{c}}{(\mathfrak{q}, \mathfrak{c})}\right) k^{d b}$, with a constant $b \in(-1,0)$ to be chosen later. 
Case II.i $N\left(n \frac{(c, q)}{q}+|\epsilon|^{2} m_{2}+2 \sqrt{n m_{2}}|\epsilon|\left(\frac{(c, q)}{q}\right)^{\frac{1}{2}} \sin \underline{\theta}\right)>N\left(\frac{\mathfrak{q} \mathfrak{c}}{(\mathfrak{q}, \mathfrak{c})}\right) k^{d b}$

In this case we apply integration by parts to the integral over $\gamma$. Therefore we consider the derivatives of $\mathcal{V}(\underline{\gamma})$. Let $\underline{\alpha} \in \mathbb{N}_{0}^{d}$, and denote by $\frac{d^{\underline{\alpha}}}{d \underline{\underline{\alpha}}}$ and $f^{(\underline{\alpha})}(\underline{\gamma})$ the $\alpha_{j}$-fold derivative in the $j$-th variable, $j=1, \ldots, d$. Then

$$
\begin{aligned}
\frac{d^{\underline{\alpha}}}{d \underline{\gamma} \underline{\alpha}} \mathcal{V}(\underline{\gamma}) & =\sum_{\underline{\mu}+\underline{\nu}+\underline{o}=\underline{\alpha}} C(\underline{\mu}, \underline{\nu}, \underline{o}) \widehat{\mathcal{V}}^{(\underline{\nu})}\left(\frac{1}{c^{2} \underline{\gamma}}\right)\left(\frac{1}{N \underline{\gamma}}\right)^{(\underline{\mu})} \Psi^{(\underline{o})}(N \underline{\gamma}) \\
& \ll \sum_{\substack{\underline{\mu}+\underline{\nu}+\underline{o}=\underline{\alpha} \\
\kappa_{j}=0, \ldots, \nu_{j}}} M_{1 i}^{\frac{1}{2}}\left(c^{-2} M_{1 i}\right)^{\underline{\kappa}}|\underline{\gamma}|^{-(\underline{\alpha}+\underline{\kappa}+1)} \Psi(N \underline{\gamma})
\end{aligned}
$$

because the derivatives of $\widehat{\mathcal{V}}$ can be estimated via the chain rule.

Now we apply integration by parts to the $\underline{\gamma}$ integral $\left\lceil\alpha_{j}+\eta_{j}\right\rceil$-times in the $j$-th variable, $j=1, \ldots, d$, where $0<\eta_{j}=\eta<\frac{1}{2}$ if $\left|\epsilon_{j}\right|>1$ and zero else. We choose $\alpha_{j}=\alpha$ for all $j$.

Thus, we obtain

$$
\begin{aligned}
& \int_{(0, \infty))^{d}} e\left[\left(n \frac{(c, q)}{q}+|\epsilon|^{2} m_{2}+2 \sqrt{n m_{2}}|\epsilon|\left(\frac{(c, q)}{q}\right)^{\frac{1}{2}} \sin \underline{\theta}\right) \underline{\gamma}\right] \mathcal{V}(\underline{\gamma}) d \underline{\gamma} \\
& \ll \sum_{\substack{\mu_{j}+\nu_{j}+o_{j}=\alpha+\eta \\
\kappa=0, \ldots, \nu_{j} \\
j=1, \ldots d}} M_{1 i}^{\frac{1}{2}+\underline{\kappa}} c^{-2 \underline{\kappa}} \int_{\substack{\frac{1}{4} \\
M_{1 i}^{1-\varepsilon}(N c)^{-2} \leq N \underline{\gamma} \leq k^{4 d}}}(N \underline{\gamma})^{-\alpha-1} \Psi(N \underline{\gamma}) \underline{\gamma}^{-\underline{\kappa}} d \underline{\gamma} \times \\
& \times N\left(n \frac{(c, q)}{q}+|\epsilon|^{2} m_{2}+2 \sqrt{n m_{2}}|\epsilon|\left(\frac{(c, q)}{q}\right)^{\frac{1}{2}} \sin \underline{\theta}\right)^{-\alpha-\eta_{j}} \\
& \ll N\left(n \frac{(c, q)}{q}+|\epsilon|^{2} m_{2}+2 \sqrt{n m_{2}}|\epsilon|\left(\frac{(c, q)}{q}\right)^{\frac{1}{2}} \sin \underline{\theta}\right)^{-\alpha-\eta_{j}} \sum_{\substack{\mu_{j}+\nu_{j}+o_{j}=\alpha+\eta \\
\kappa=0, \ldots, \nu_{j} \\
j=1, \ldots d}} M_{1 i}^{\frac{1}{2}+\underline{\kappa}}\left(M_{1 i}^{1-\varepsilon} \frac{1}{4}\right)^{-(\alpha+\eta+\underline{\kappa})}(N c)^{2(\alpha+\eta)} \\
& \ll \prod_{\left|\epsilon_{j}\right|>1}\left|\epsilon_{j}\right|^{-\eta}\left(N\left(n \frac{(c, q)}{q|\epsilon|}+|\epsilon| m_{2}+2 \sqrt{n m_{2}}\left(\frac{(c, q)}{q}\right)^{\frac{1}{2}} \sin \underline{\theta}\right)\right)^{-\alpha-\eta}(N c)^{2(\alpha+\eta)} M_{1 i}^{\frac{1}{2}-\alpha(1-\varepsilon)} .
\end{aligned}
$$

Next, we sum over $n, m_{2}, c$ and $\epsilon$. The Ramanujan sum $r_{c}\left(\epsilon^{2} m_{2}-n\left(\frac{q}{(c, q)}\right)^{*}\right)$ is trivially bounded by Euler's $\varphi$-function $\varphi(c)$.

Then summing over $n$ with $N n<\left(N m_{2}\right)^{1+\varepsilon}$ and making use of the lower bound for the factor $N\left(n \frac{(c, q)}{q|\epsilon|}+|\epsilon| m_{2}+2 \sqrt{n m_{2}}\left(\frac{(c, q)}{q}\right)^{\frac{1}{2}} \sin \underline{\theta}\right)$ we obtain

$$
\begin{aligned}
& \sum_{\substack{N n<\left(N m_{2}\right)^{1+\varepsilon} \\
\ll}} C_{g}((n))\left(N\left(n \frac{(c, q)}{q|\epsilon|}+|\epsilon| m_{2}+2 \sqrt{n m_{2}}\left(\frac{(c, q)}{q}\right)^{\frac{1}{2}} \sin \underline{\theta}\right)\right)^{-\alpha-\eta} \\
& \ll\left(N \frac{\mathfrak{q} \mathfrak{c}}{(\mathfrak{q}, \mathfrak{c})}\right)^{-\alpha-\eta} k^{-(\alpha+\eta) d b}\left(N m_{2}\right)^{1+2 \varepsilon} .
\end{aligned}
$$

Thus, the sum over $m_{2}$ and $n$ is dominated by

$$
\begin{aligned}
& \sum_{m_{2}} \phi_{j}\left(\frac{N m_{2}}{2^{j}}\right) \frac{\left|C_{g}\left(\mathfrak{m}_{2}\right)\right|}{\left(N m_{2}\right)^{\frac{1}{2}}} V_{\frac{1}{2}}\left(\frac{N\left(\mathfrak{b}_{2} \mathfrak{a}_{2}^{2} \mathfrak{m}_{2}\right)}{D_{K}^{2} N\left(\frac{\mathfrak{q} \mathfrak{c}}{(\mathfrak{q}, \mathfrak{c})}\right)}\right)\left(N \frac{\mathfrak{q} \mathfrak{c}}{(\mathfrak{q}, \mathfrak{c})}\right)^{-\alpha-\eta} k^{-(\alpha+\eta) d b}\left(N m_{2}\right)^{1+2 \varepsilon} \\
& \ll\left(N \frac{\mathfrak{q} \mathfrak{c}}{(\mathfrak{q}, \mathfrak{c})}\right)^{-\alpha-\eta} k^{-(\alpha+\eta) d b} M_{2 j}^{\frac{3}{2}+3 \varepsilon} .
\end{aligned}
$$

As above, the sum over the units $\epsilon$ is $\mathcal{O}(1)$. The integral over $\underline{\theta}$ is trivially bounded by $(2 \pi)^{d}$, and using trivial estimates for the sum over $c$, we obtain that the expression (4.2.17), restricted to 
$N\left(n \frac{(c, q)}{q}+|\epsilon|^{2} m_{2}+2 \sqrt{n m_{2}}|\epsilon|\left(\frac{(c, q)}{q}\right)^{\frac{1}{2}} \sin \underline{\theta}\right)>N\left(\frac{\mathfrak{q} \mathfrak{c}}{(\mathfrak{q}, \mathfrak{c})}\right) k^{d b}$, is bounded by

$$
\ll\left(N \frac{\mathfrak{q} \mathfrak{c}}{(\mathfrak{q}, \mathfrak{c})}\right)^{-\alpha-\eta} k^{-(\alpha+\eta) d b} M_{2 j}^{\frac{3}{2}+3 \varepsilon} M_{1 i}^{\frac{1}{2}-\alpha(1-\varepsilon)} \frac{Q_{l}^{2(\alpha+\eta)}}{N \mathfrak{c}} .
$$

Case II.ii $N\left(n \frac{(c, q)}{q}+|\epsilon|^{2} m_{2}+2 \sqrt{n m_{2}}|\epsilon|\left(\frac{(c, q)}{q}\right)^{\frac{1}{2}} \sin \underline{\theta}\right) \leq N\left(\frac{\mathfrak{q} \mathfrak{c}}{(\mathfrak{q}, \mathfrak{c})}\right) k^{d b}$

We use quadratic extension to rewrite

$$
\begin{aligned}
& N\left(n \frac{(c, q)}{q}+|\epsilon|^{2} m_{2}+2 \sqrt{n m_{2}}|\epsilon|\left(\frac{(c, q)}{q}\right)^{\frac{1}{2}} \sin \underline{\theta}\right) \\
= & N\left(\left(\sqrt{n}\left(\frac{(c, q)}{q}\right)^{\frac{1}{2}}-\sqrt{m_{2}}|\epsilon|\right)^{2}+2 \sqrt{n m_{2}}|\epsilon|\left(\frac{(c, q)}{q}\right)^{\frac{1}{2}}(1+\sin \underline{\theta})\right) .
\end{aligned}
$$

Note that both summands are non-negative. Thus, we extend the sum if we replace the conditions on $n, m_{2}, \epsilon$ and $\underline{\theta}$ by the conditions

$$
\begin{aligned}
N\left(\sqrt{n}\left(\frac{(c, q)}{q}\right)^{\frac{1}{2}}-\sqrt{m_{2}}|\epsilon|\right)^{2} & \leq\left(N \frac{\mathfrak{q} \mathfrak{c}}{(\mathfrak{q}, \mathfrak{c})}\right) k^{d b}, \\
|N(1+\sin \underline{\theta})| & <k^{d(b-1)} .
\end{aligned}
$$

The condition on $\underline{\theta}$ is equivalent to

$$
\left|\frac{\pi}{2}+\theta_{j}\right|<k^{\frac{b-1}{2}}
$$

as $\theta_{j} \in(-\pi, \pi)$ for all $j$.

We have to estimate

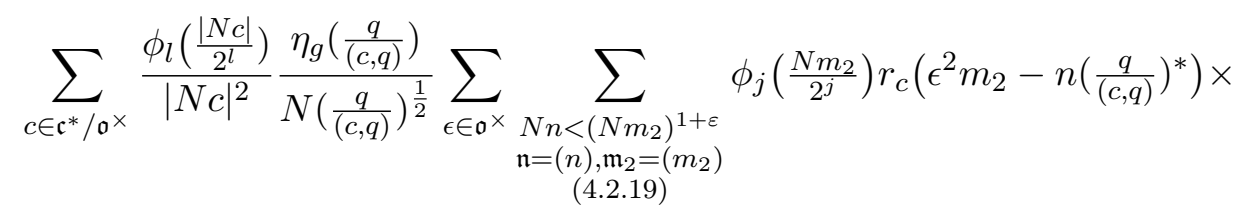

$$
\begin{aligned}
& \times \frac{C_{g}(\mathfrak{n}) C_{g}\left(\mathfrak{m}_{2}\right)}{\left(N m_{2}\right)^{\frac{1}{2}}} V_{\frac{1}{2}}\left(\frac{N\left(\mathfrak{b}_{2} \mathfrak{a}_{2}^{2} \mathfrak{m}_{2}\right)}{D_{K}^{2} N\left(\frac{\mathfrak{q} \mathfrak{c}}{(\mathfrak{q}, \mathfrak{c})}\right)}\right) \times \\
& \times \frac{1}{\pi^{d}} \int_{[4.2 .20} \ldots \iint_{(0, \infty)^{d}} \ldots \int\left|e^{-i(k-1) \operatorname{Tr}(\underline{\theta})} e\left[\left(n \frac{(c, q)}{q}+|\epsilon|^{2} m_{2}+2 \sqrt{n m_{2}}|\epsilon|\left(\frac{(c, q)}{q}\right)^{\frac{1}{2}} \sin \underline{\theta}\right) \underline{\gamma}\right] \mathcal{V}(\underline{\gamma})\right| d \underline{\gamma} d \underline{\theta} \text {. }
\end{aligned}
$$

To begin with we estimate the two multiple-integrals.

Recall that $\mathcal{V}$ is a product of the three functions $\widehat{\mathcal{V}}\left(\frac{1}{c^{2} \underline{\gamma}}\right), \frac{1}{N \underline{\gamma}}$ and $\Psi(N \underline{\gamma})$. Using the trivial estimate 4.2.14 for $\widehat{\mathcal{V}}$ and the support property of $\Psi$ we obtain that the $\underline{\gamma}$-integral is bounded by

$$
\ll \sqrt{M_{1 i}} \log \left(N \mathfrak{c} k^{d}\right)
$$

The $\underline{\theta}$-integral over the domain 4.2 .20 can trivially be bounded by

$$
\ll 2^{d} k^{d \frac{b-1}{2}} .
$$

We are left with the summation over $\mathfrak{n}, \mathfrak{m}_{2}, c$ and $\epsilon$. By the condition 4.2.19) and our choice of the fundamental domain $\mathcal{F}$ we obtain that $\left|\epsilon_{j}\right|<\left(N \frac{\mathfrak{q} \mathfrak{c}}{(\mathfrak{q}, \mathfrak{c})}\right)^{\frac{1}{2 d}} k^{\frac{b}{2}}$ for all $j=1, \ldots, d$.

Now, we write $n \frac{(c, q)}{q}=|\epsilon|^{2} m_{2}+h$ with $h \in \mathcal{F}$ as $n, m_{2} \in \mathcal{F}$ to change the summation condition 4.2.19) into $N h \leq\left(N \frac{\mathfrak{q} \mathfrak{c}}{(\mathfrak{q}, \mathfrak{c})}\right)^{\frac{1}{2}} k^{d \frac{b}{2}} \sqrt{m_{2}}$. 
We estimate the Fourier coefficients by 4.1.3.

Thus, the sum over $h$ and $m_{2}$ is bounded by

$$
\sum_{\substack{h, m_{2} \in \mathcal{F} \\ \mathfrak{m}_{2}=\left(m_{2}\right)}} \phi_{j}\left(\frac{N m_{2}}{2^{j}}\right) r_{c}(|h|) \frac{\left|C_{g}\left(\left(m_{2}|\epsilon|^{2}+h\right)\right) C_{g}\left(\mathfrak{m}_{2}\right)\right|}{\left(N m_{2}\right)^{\frac{1}{2}}} V_{\frac{1}{2}}\left(\frac{N\left(\mathfrak{b}_{2} \mathfrak{a}_{2}^{2} \mathfrak{m}_{2}\right)}{D_{K}^{2} N\left(\frac{\mathfrak{q} \mathfrak{c}}{(\mathfrak{q}, \mathfrak{c})}\right)}\right) \ll M_{2 j}^{1+2 \varepsilon}\left(N \frac{\mathfrak{q} \mathfrak{c}}{(\mathfrak{q}, \mathfrak{c})}\right)^{\frac{1}{2}} k^{d \frac{b}{2}}
$$

As a consequence, the sum over $\epsilon$ is bounded by

$$
\sum_{\substack{\epsilon \in \mathfrak{o}^{\times} \\\left|\epsilon_{j}\right|<\left(N \frac{\mathfrak{q} \mathfrak{c}}{(\mathfrak{q}, \mathfrak{c})}\right)^{\frac{1}{2 d}}}} 1 \ll 1 .
$$

Finally we sum over $c \in \mathfrak{c}^{*} / \mathfrak{o}^{\times}$to see that 4.2.21 can be estimated by

$$
\ll \frac{Q_{l}}{(N \mathfrak{c})^{3}}\left(N \frac{\mathfrak{q} \mathfrak{c}}{(\mathfrak{q}, \mathfrak{c})}\right)^{\frac{1}{2}+\varepsilon} k^{d\left(b-\frac{1}{2}+\varepsilon\right)} \sqrt{M_{1 i}} M_{2 j}^{1+2 \varepsilon} .
$$

Combining the bounds 4.2 .18 and 4.2 .22 we get

$$
M_{i j l}^{\Psi} \ll\left(N \frac{\mathfrak{q} \mathfrak{c}}{(\mathfrak{q}, \mathfrak{c})}\right)^{-\alpha-\eta} k^{-(\alpha+\eta) d b} M_{2 j}^{\frac{3}{2}+3 \varepsilon} M_{1 i}^{\frac{1}{2}-\alpha(1-\varepsilon)} \frac{Q_{l}^{2(\alpha+\eta)}}{N \mathfrak{c}}+\frac{Q_{l}}{(N \mathfrak{c})^{3}}\left(N \frac{\mathfrak{q} \mathfrak{c}}{(\mathfrak{q}, \mathfrak{c})}\right)^{\frac{1}{2}+\varepsilon} k^{d\left(b-\frac{1}{2}+\varepsilon\right)} \sqrt{M_{1 i}} M_{2 j}^{1+2 \varepsilon} .
$$

Thus, we are left with the summation over $(i, j, l) \in C$. We recall that $\eta<\frac{1}{2}$. Considering the definition 4.2 .9 of $C$, we have on $Q_{l}$ the upper bound $Q_{l} \ll \sqrt{M_{1 i} M_{2 j}} k^{-d}$ which implies that $M_{1 i}$ is bounded by $M_{1 i}^{-\alpha(1-\varepsilon)+\frac{1}{2}}<\left(M_{2 j}\left(k^{d} Q_{l}\right)^{-2}\right)^{\alpha-\frac{1}{2}}$. The sum over $i$ contains $\log \left(k^{d} N \frac{\mathfrak{q} \mathfrak{c}}{(\mathfrak{q}, \mathfrak{c})}\right)$ elements. If the exponent is positive the upper bounds for $M_{*}$ are given in 4.2 .9 . Further we use $Q_{l}<\left(N \frac{\mathfrak{q} \mathfrak{c}}{(\mathfrak{q}, \mathfrak{c})}\right) k^{d \varepsilon}$ to obtain

$$
\begin{aligned}
\sum_{(i, j, l) \in C}\left|M_{i j l}^{\Psi}\right| \ll & \left(N \frac{\mathfrak{q} \mathfrak{c}}{(\mathfrak{q}, \mathfrak{c})}\right)^{-\alpha \varepsilon+\frac{3}{2}+6 \varepsilon} k^{d(-\alpha(b+1)+2+9 \varepsilon)}\left(N\left(\mathfrak{b}_{2} \mathfrak{a}_{2}^{2}\right)\right)^{-1-\frac{\alpha}{2}} \\
& +\left(N \frac{\mathfrak{q} \mathfrak{c}}{(\mathfrak{q}, \mathfrak{c})}\right)^{6 \varepsilon} k^{d(b+1+9 \varepsilon)} N\left(\frac{\mathfrak{q}}{(\mathfrak{q}, \mathfrak{c})}\right)\left(N\left(\mathfrak{b}_{1} \mathfrak{a}_{1}^{2}\right)\right)^{-\frac{1}{2}}\left(N\left(\mathfrak{b}_{2} \mathfrak{a}_{2}^{2}\right)\right)^{-1} \\
\ll & (N \mathfrak{c})^{\frac{1}{2}+3 \varepsilon} k^{-\frac{1}{2}+\varepsilon}+\left(N \frac{\mathfrak{q} \mathfrak{c}}{(\mathfrak{q}, \mathfrak{c})}\right)^{6 \varepsilon}\left(N \frac{\mathfrak{q}}{(\mathfrak{q}, \mathfrak{c})}\right)^{3 \varepsilon} k^{9 d \varepsilon} .
\end{aligned}
$$

The second estimate follows by choosing $b=-1+3 \varepsilon$ and $\alpha=\left\lceil\frac{1}{\varepsilon}\right\rceil$.

The sums over $\mathfrak{a}_{\iota}$ and $\mathfrak{b}_{\iota}$ are bounded by

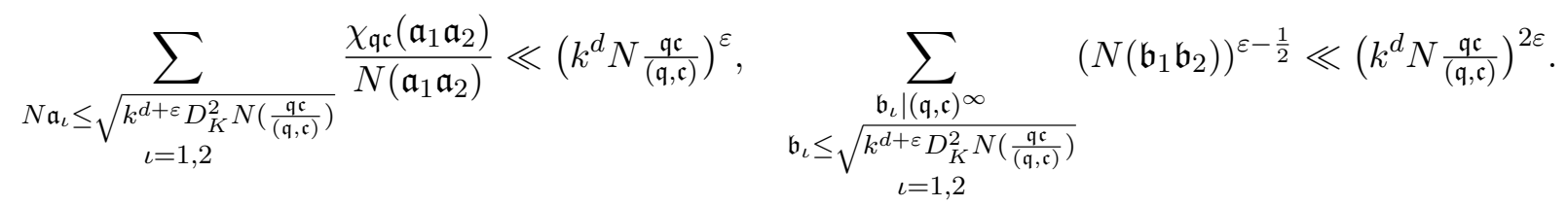

Combining the results 4.2.8 with 4.2.12, 4.2.16 and 4.2.23 we obtain

$$
M_{S}(g) \ll k^{d 15 \varepsilon}(N \mathfrak{c})^{9 \varepsilon}\left(N \frac{\mathfrak{q}}{(\mathfrak{q}, \mathfrak{c})}\right)^{6 \varepsilon} .
$$

Then by 4.2.3 , 4.2.4 and 4.2.25 Theorem 1.2.1 is follows. 


\section{Chapter 5}

\section{Appendix}

\subsection{Whittaker Functions}

The Whittaker function is solution of a differential equation. More precisely, see [GR07, 9.220], the equation

$$
\frac{d^{2} W}{d z^{2}}+\left(-\frac{1}{4}+\frac{a}{z}+\frac{\frac{1}{4}-b^{2}}{z^{2}}\right) W=0
$$

has the two linearly independent solutions

$$
\begin{aligned}
M_{a, b}(z) & =z^{b+\frac{1}{2}} e^{-\frac{z}{2}} \Phi\left(b-a+\frac{1}{2}, 2 b+1 ; z\right), \\
M_{a,-b}(z) & =z^{-b+\frac{1}{2}} e^{-\frac{z}{2}} \Phi\left(-b-a+\frac{1}{2},-2 b+1 ; z\right) .
\end{aligned}
$$

Then the Whittaker function is given by

$$
W_{a, b}(z)=\frac{\Gamma(-2 b)}{\Gamma\left(\frac{1}{2}-b-a\right)} M_{a, b}(z)+\frac{\Gamma(2 b)}{\Gamma\left(\frac{1}{2}+b-a\right)} M_{a,-b}(z) .
$$

\subsection{Bessel Functions}

The Bessel functions are solutions of the differential equation

$$
\frac{d^{2} Z_{\nu}}{d z^{2}}+\frac{1}{z} \frac{d Z_{\nu}}{d z}+\left(1-\frac{\nu^{2}}{z^{2}}\right) Z_{\nu}=0
$$

for $\nu \in \mathbb{C}$, see [GR07, 8.40]. For $|\arg z|<\pi$ the Bessel function of the first kind $J_{\nu}(z)$ is given by the series

$$
J_{\nu}(z)=\frac{z^{\nu}}{2^{\nu}} \sum_{k=0}^{\infty}(-1)^{k} \frac{z^{2 k}}{2^{2 k} k ! \Gamma(\nu+k+1)} .
$$

The modified Bessel function $I_{\nu}$ is defined as

$$
I_{\nu}(z)= \begin{cases}e^{-\frac{1}{2} i \pi \nu} J_{\nu}\left(e^{\frac{i \pi}{2}} z\right), & \text { for }-\pi<\arg z \leq \frac{\pi}{2} \\ e^{-\frac{3}{2} i \pi \nu} J_{\nu}\left(e^{\frac{3 i \pi}{2}} z\right), & \text { for } \frac{\pi}{2}<\arg z \leq \pi\end{cases}
$$


We write $J_{\nu}^{+}$to denote $J_{\nu}$ and $J_{\nu}^{-}$for the modified Bessel function $I_{\nu}$.

The modified Bessel $K$-function is defined as

$$
K_{\nu}(z)=\left\{\begin{array}{l}
\frac{\pi i}{2} e^{\frac{\pi}{2} i \nu}\left(J_{\nu}(i z)+i Y_{\nu}(i z)\right), \quad \text { for }-\pi<\arg z \leq \frac{\pi}{2} \\
\frac{-\pi i}{2} e^{-\frac{\pi}{2} i \nu}\left(J_{-\nu}(-i z)-i Y_{-\nu}(-i z)\right), \quad \text { for } \frac{\pi}{2}<\arg z \leq \pi
\end{array}\right.
$$

where $Y_{\nu}(z)=\frac{1}{\sin (\pi \nu)}\left[\cos (\pi \nu) J_{\nu}(z)-J_{-\nu}(z)\right]$. 
List of Symbols

\begin{tabular}{|c|c|}
\hline Symbol & Pag \\
\hline$\lambda_{\underline{\nu}}(\mathfrak{n})$ & 10 \\
\hline$\Lambda^{d-1}$ & 10 \\
\hline$\zeta_{K}(s, \underline{\nu})$ & 11 \\
\hline$\chi_{\mathfrak{q} \mathfrak{c}}(\mathfrak{n})$ & 59 \\
\hline$L\left(s, \chi_{\mathfrak{q} \mathfrak{c}}\right)$ & 59 \\
\hline$L(f \otimes g, s)$ & 59 \\
\hline$e[x]$ & 9 \\
\hline$S_{K}(m, n ; c)$ & 9 \\
\hline$\Psi(x)$ & 13 \\
\hline$\Psi_{+}(x)$ & 13 \\
\hline$c_{\Psi}$ & 27 \\
\hline $\mathrm{n}[\underline{x}]$ & 14 \\
\hline $\mathrm{a}[\underline{y}]$ & 14 \\
\hline $\mathrm{k}[\underline{\theta}]$ & 14 \\
\hline $\mathrm{w}_{j}$ & 14 \\
\hline $\mathrm{e}_{j}^{ \pm}$ & 14 \\
\hline$\Omega_{j}$ & 14 \\
\hline$\psi_{n}(\mathrm{~g})$ & 15 \\
\hline$E_{q}(\mathrm{~g} ; s, \underline{\nu})$ & 15 \\
\hline$\kappa_{V}$ & 18 \\
\hline$\varrho_{V}$ & 21 \\
\hline$\eta_{V}(\cdot)$ & 21 \\
\hline$a_{V}$ & 21 \\
\hline $\mathrm{B}_{\mathrm{e}} f$ & 22 \\
\hline$H_{V}(s)$ & 23 \\
\hline $\mathcal{Z}(g, \omega ; K)$ & 26 \\
\hline$C_{K}(g, \omega)$ & 26 \\
\hline $\mathcal{J}(\underline{z} ; g, \omega)$ & 26 \\
\hline $\mathcal{J}_{0}(\underline{z} ; g, \omega)$ & 27 \\
\hline $\mathcal{J}_{+}(\underline{z} ; g, \omega)$ & 27 \\
\hline $\mathcal{J}^{(r)}(\underline{z} ; g, \omega)$ & 46 \\
\hline$W(\underline{x})$ & 27 \\
\hline$W_{+}(\underline{x} ; \gamma, \delta)$ & 28 \\
\hline$W_{+}(\underline{s} ; \mathrm{e} ; \gamma, \delta)$ & 42 \\
\hline$\ddot{W}_{+}\left(s_{1}, s_{2} ; z_{1}, z_{3}\right)$ & 45 \\
\hline $\mathcal{B}_{m}(\alpha, \beta ; h)$ & 27 \\
\hline$D_{m}\left(\alpha, \frac{a}{c} ; h\right)$ & 28 \\
\hline $\mathrm{D}_{\ell}\left(s, \alpha ; \underline{\nu} ; \frac{a}{c}\right)$ & 30 \\
\hline
\end{tabular}

\begin{tabular}{|c|c|}
\hline Symbol & Page \\
\hline $\operatorname{sgn}^{\ell}[n]$ & 30 \\
\hline$\Gamma_{\ell}(\underline{s} ; \alpha)$ & 30 \\
\hline$h(\underline{x})$ & 27 \\
\hline$\widetilde{h}(\underline{s} ; \mathrm{e})$ & 29 \\
\hline$\widetilde{h}_{\ell}$ & 30 \\
\hline$\ddot{h}\left(\eta_{1}, \eta_{2}\right)$ & 39 \\
\hline$[h]_{\ell}(x ; \alpha)$ & 33 \\
\hline$[[h]]_{\ell}(x ; \alpha, \beta ; \xi)$ & 34 \\
\hline$S_{m, n}\left(\alpha, \beta, \xi ;[h]_{\ell}\right)$ & 34 \\
\hline$\Delta_{e_{j}}^{ \pm}$ & 37 \\
\hline$\Phi_{*}(\underline{r} ; \alpha, \beta ; h)$ & 37 \\
\hline$\Psi_{t}(\alpha, \beta ; h)$ & 40 \\
\hline$\Lambda_{V}(g, \omega)$ & 47 \\
\hline$\Xi_{\underline{\nu}}(t ; g, \omega)$ & 47 \\
\hline$M_{K}(g, \omega)$ & 47 \\
\hline$\left.f\right|_{\gamma}$ & 56 \\
\hline$\left\langle f_{1}, f_{2}\right\rangle$ & 57 \\
\hline$W_{q}$ & 57 \\
\hline$\eta_{g}(q)$ & 57 \\
\hline$C_{g}(\mathfrak{n})$ & 57 \\
\hline $\mathbf{J}_{\underline{k}}(x)$ & 64 \\
\hline$M^{\sharp}(g)$ & 66 \\
\hline$M(g)$ & 66 \\
\hline$M_{\delta}$ & 66 \\
\hline$M_{S}$ & 66 \\
\hline$M_{1 i}$ & 68 \\
\hline$M_{2 j}$ & 68 \\
\hline$Q_{l}$ & 68 \\
\hline$\phi_{*}$ & 68 \\
\hline$M_{i j l}$ & 68 \\
\hline$M_{i j l}^{2}$ & 70 \\
\hline$M_{i j l}^{<}$ & 70 \\
\hline$M_{i j l}^{\Psi}$ & 73 \\
\hline $\mathcal{V}(\underline{x})$ & 72 \\
\hline$\widehat{\mathcal{V}}(1 / \underline{\gamma})$ & 72 \\
\hline $\mathcal{V}(\underline{\gamma})$ & 73 \\
\hline
\end{tabular}




\section{Bibliography}

[AL78] A. Atkin and W. Li. Twists of newforms and pseudo-eigenvalues of $W$-operators. Invent. Math. Volume 48. pages 221-243 (1978)

[BB04] J. Beineke and D. Bump. Moments of the Riemann zeta function and Eisenstein series-I. Journal of Number Theory. Volume 105. pages 150-174 (2004)

[B11] V. Blomer. Period Integrals and Rankin-Selberg L-functions on GL(n). Geometric and Functional Analysis. Volume 22. pages 608-620 (2011)

[BB11] V. Blomer and F. Brumley. On the Ramanujan Conjecture over Number Fields. arXiv:1003.0559 (2011)

[BM15] V. Blomer and D. Milićević. The Second Moment of Twisted Modular L-Functions. Geometric and Functional Analysis. Volume 25. Issue 2. pages 453-516 (2015)

[BFKMM17] V. Blomer, É. Fouvry, E. Kowalski, P. Michel and D. Milićević. On Moments of twisted L-Functions. American Journal of Mathematics. Volume 139. Number 3. pages 707-768 (2017)

[B81] R. W. Bruggeman. Fourier Coefficients of Automorphic Forms. Springer Lecture Notes in Mathematics. Volume 865 (1981)

[BM01] R. W. Bruggeman and Y. Motohashi. Fourth Power Moment of Dedekind Zeta-Functions of Real Quadratic Number Fields. Functiones et Approximatio. Volume XXIX. pages 41-79 (2001)

[BM02] R. W. Bruggeman and Y. Motohashi. Sum formula for Kloosterman sums and fourth moment of the Dedekind zeta-function over the Gaussian number field. Cambridge University Press (2002)

[BMP01] R. W. Bruggeman, R. J. Miatello and I.Pacharoni. Estimates for Kloosterman sums for totally real number fields. Journal für die reine und angewandte Mathematik. Volume 535. pages 103-164 (2001)

[C90] M. Coleman. A zero-free region for the Hecke L-functions. Mathematika, Volume 37(2), pages 287-304. (1990)

[C92] M. Coleman. The distribution of points at which norms are prime. Journal of Number Theory. Volume 4. pages 359-378 (1992) 
[DG09] A. Diaconu and P. Garrett. Integral moments of automorphic L-functions. Cambridge University Press. Journal of the Institute of Mathematics of Jussieu. Volume 8. Issue 2. pages 335-382 (2009)

[DGG11] A. Diaconu, P. Garrett and D. Goldfeld. Moments for L-functions for $G L_{r} \times G L_{r-1}$. Springer Proceedings in Mathematics. Volume 9. pages 197-227 (2011)

[D89] W. Duke. Some problems in multidimensional analytic number theory. Acta Arithmetica. Volume LII. pages 203-228 (1989)

[GR07] I. S. Gradshteyn and I. M. Ryzhik. Table of Integrals, Series, and Products. Seventh Edition. Editors A. Jeffrey and D. Zwillinger. Academic Press (2007)

[HB79] D. R. Heath-Brown. The Fourth Power Moment of the Riemann Zeta Function. Proceedings of the London Mathematical Society. Volume s3-38. Issue 3. pages 385-422 (1979)

[H12] W. Heap. The Twisted Second Moment of the Dedekind Zeta Function of a Quadratic Field. International Journal of Number Theory. Volume 10. (2012)

[H19] W. Heap. Moments of the Dedekind zeta function and other non-primitive L-functions. Cambridge University Press. Mathematical Proceedings of the Cambridge Philosophical Society. pages 1-29 (2019)

[HT16] A. Hamieh and N. Tanabe. Determining Hilbert Modular Forms by Central Values of Rankin-Selberg Convolutions: The Level Aspect. arXiv:1609.07209 (2016)

[HT20] A. Hamieh and N. Tanabe. Non-vanishing of Rankin-Selberg convolutions for Hilbert modular forms. Mathematische Zeitschrift. (2020)

[I28] A. E. Ingham. Mean-Value Theorems in the Theory of the Riemann Zeta-Function. Proceedings of the London Mathematical Society. Volume s2-27. Issue 1. pages 273-300 (1928)

[IL07] H. Iwaniec and X. Li. The orthogonality of Hecke eigenvalues. Compositio Math. Volume 143. pages 541-565 (2007)

[JL70] H. Jacquet and R.P. Langlands, Automorphic Forms on GL(2). Springer Lecture Notes in Mathematics. Volume 114. pages 1-548 (1970)

[KMV00] E. Kowalski, P. Michel and J. Vanderkam. Rankin-Selberg $L$-functions in the level aspect. Duke Mathematical Journal. Volume 114. No 1. pages 123-191.(2000)

[L90] R. Laun. Darstellung total positiver ganzer algebraischer Zahlen als Summe $N$-freier Zahlen. Acta Arithmetica. Volume 55. pages 171-190 (1990)

[L79] W.-C. W. Li. L-series of Rankin type and their functional equation. Math. Ann., Vol. 244, pages 135-166, (1979)

[LM14] S.-C. Liu and R. Masri. Nonvanishing of Rankin-Selberg $L$-functions for Hilbert modular forms. Ramanujan Journal. Volume 34.pages 227-236 (2014) 
[L03] W. Luo. Poincaré Series and Hilbert Modular Forms. The Ramanujan Journal. Volume 7. pages 129-140 (2003)

[KS02] H. Kim and F. Shahidi. Cuspidality of symmetric Powers with Applications. Duke Mathematical Journal. Volume 112. No. 1. pages 177-197 (2002)

[M97] Y. Motohashi. Spectral Theory of the Riemann Zeta-Function. Cambridge University Press (1997)

[M07] Y. Motohashi. The Riemann Zeta-Function and Hecke Congruence Subgroups II. arXiv:0709.2590 (2007)

[N20] P. Nelson. Eisenstein series and the cubic moment for PGL(2). arXiv:1911.06310 (2020)

[S78] G. Shimura. The special values of the zeta functions associated with Hilbert modular forms. Duke Mathematikal Journal. Volume 45. pages 637-679 (1978)

[S97] P. Söhne. An Upper Bound for Hecke Zeta-Functions with Groessencharacters. Journal of Number Theory. Volume 66. Issue 2. pages 225-250 (1997)

[Th19] J. Thorner. Bounded gaps between primes in multidimensional Hecke equidistribution problems. Mathematical Research Letters. Volume 26. Number 3. pages 875-901 (2019)

[T51] E.C. Titchmarsh. The theory of the Riemann zeta function. Oxford science publications. Clarendon Press. (1951)

[To19] B. Topacogullari. The fourth moment of individual Dirichlet L-functions on the critical line. arXiv:1909.11517 (2019)

[W13] N. Watt. Weighted fourth moments of Hecke zeta functions with groessencharacters. arXiv:1307.5333 (2013)

[Y06] M. Young. The fourth moment of Dirichlet L-functions. Annals of Mathematics. Volume 173. Issue 1. pages $1-50(2006)$ 